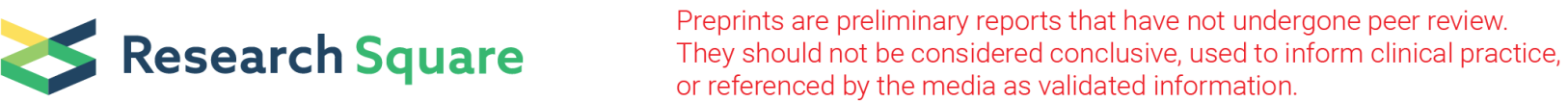

\section{Post-stroke Delivery of Valproic acid Promotes Functional Recovery and Differentially Modifies Responses of Peri-infarct Microglia}

\section{Tung-Tai Kuo}

Tri-Service General Hospital Department of Neurology Surgery https://orcid.org/0000-0001-9513-0358

\section{Yuan-Hao Chen}

Tri-Service General Hospital Department of Neurology Surgery,National Defense Medical Center

\section{Vicki Wang}

National Defense Medical Center Graduate Institute of Medical Sciences

Jui-Sheng Wu

National Defense Medical Center Department of Biology and Anatomy

Kuan-Yin Tseng ( $\nabla$ neuronsurgery@gmail.com )

Tri-Service General Hospital, National Defense Medical Center

\section{Research}

Keywords: distal middle cerebral artery occlusion (dMCAo), valproic acid (VPA), galectin-3, microglia activation

Posted Date: December 1st, 2020

DOl: https://doi.org/10.21203/rs.3.rs-115994/v1

License: (c) (i) This work is licensed under a Creative Commons Attribution 4.0 International License. Read Full License 


\section{Abstract}

Background: Recently, microglia, being the determinant of environment in peri-infarct tissue and strongly influence the potential for neuronal plasticity, has been implicated in post-ischemic secondary injury and functional recovery. However, the specific role of peri-infarct microglia and the timing of its morphological changes following ischemic stroke are not well understood. Valproic acid (VPA) can protect against ischemic damage and promote recovery. These effects are usually attributed, at least partially, to the antiinflammatory ability of this drug to suppress microglial activation. In this study, we explored whether a low dose of VPA after stroke could modify reactive responses in microglia/macrophages and optimize peri-infarct microenvironments to improve functional recovery.

Methods: Male Sprague-Dawley rats were subjected to distal middle cerebral artery occlusion (dMCAo) for 90 minutes, followed by reperfusion. Some received a single injection of VPA ( $200 \mathrm{mg} / \mathrm{kg}) 90$ minutes after the induction of ischemia, while vehicle-treated animals underwent the same procedure with physiological saline. Infarction volume was calculated at 48 hours after reperfusion, and neurological symptoms were evaluated through 48 hours thereafter. The production of cytokines and biomarkers after insult was determined using enzyme-linked immunosorbent assays (ELISAs) and western blot. The effects of VPA on the activation of peri-infarct CD11b-positive cells were assessed based on cellular density and quality observation of morphology with fractal analysis and circularity index. The expressions of genes within peri-infarct zones at 3 days were determined by RNA-sequencing analysis. To determine whether VPA modulates microglial polarization, gentleMACS Dissociator was used to isolate CD11b-positive cells from the peri-infarct cortex of rats treated with or without VPA 3 days after dMCAo, after which the cells were subjected to qPCR analysis.

Results: $200 \mathrm{mg} / \mathrm{kg}$ of VPA injected 90 minutes after ischemia induction did not significantly reduce infarct volume but did improve neurological deficit at least partially compared with vehicle. Meanwhile, VPA significantly reduced dMCAo-induced elevation of IL-6 at 24 hours post-stroke and significantly decreased the number of CD11b-positive microglia/macrophages within peri-infarct cortex at 7 but not at 2 days. Morphological analysis revealed that VPA therapy leads to higher fractal dimensions and lower circularity index of CD11b-positive cells within peri-infarct cortex at both 2 days and 7 days, suggesting that VPA has core effects on microglial activation. The attenuation of microglia/macrophage activation caused by VPA might involve HDAC inhibition-mediated suppression of galectin-3 production. Analysis of VPA-induced changes to gene expressions in the peri-infarct cortex at 3 days post-stroke indicated the upregulation of wound healing, collagen trimmer, and extracellular matrix genes. Furthermore, qPCR analysis of CD11b-positive cells suggested that VPA could partially enhance M2 subset polarization of microglia/macrophages in peri-infarct cortex.

Conclusions: Our results are the first to show that a low dose of VPA promotes short-term functional recovery but does not alter infarct volume. The decreases in the expression of both IL- 6 and galectin-3 might influence the extracellular matrix remodeling and morphological characteristics and transcriptional profiles of microglia/macrophages, which could contribute to the improved recovery. 


\section{Introduction}

Stroke is one of the leading causes of death and adult disability worldwide [1]. Primary ischemic stroke causes the death of neurons, astrocytes, and oligodendrocytes, as well as blood capillary damage. This destruction results in a series of pathological reactions known as secondary injuries, including blood barrier disruption and inflammatory responses [2]. Then, various types of inflammatory cells, such as T cells, macrophages, and microglia, are recruited to the lesion to remove cell debris [3]. However, these inflammatory cells elicit excessive production of cytokines and chemokines, which causes secondary cell death, expansion of the lesion region, and functional impairment of the body [4]. Most studies have focused on neuroprotection, and therefore, there is a great need to identify drug targets and develop new treatments that would improve the recovery from ischemic brain injury by targeting postischemic pathologic mechanisms such neuroinflammation [5]. The modulation of brain inflammatory cascades following stroke is recognized as a viable therapeutic strategy to promote functional recovery from ischemic brain injury. However, very few studies have taken this approach, and little is known about how recovery from stroke relates to neuroinflammation $[6,7]$.

Valproic acid (VPA), a histone deacetylase (HDAC) inhibitor, is widely used for the treatment of epileptic seizures and migraine and as a mood stabilizer in treating bipolar disorder [8]. In vitro, treatment with VPA attenuates glutamate-induced excitotoxicity, inhibits ischemia-induced fast sodium and high-voltageactivated calcium currents, and suppresses lipopolysaccharide-induced production of TNF-a and IL-6 [9]. In rat models of transient ischemia, VPA exhibits anti-inflammatory and neuroprotective effects through the inhibition of HDAC activity and induction of HSP70, which attenuates ischemia-reperfusion injury. In addition, it ameliorates blood-brain barrier (BBB) disruption through inhibition of the nuclear translocation of nuclear factor-KB activation and matrix metalloproteinase 9 production $[2,10]$. Furthermore, delayed VPA treatment could enhance white matter repair and neurogenesis in ischemic brain [11]. In an animal model of spinal cord injury, VPA was further shown to decrease purinergic $\mathrm{P} 2 \times 4$ receptor expression in activated microglia, as well as to ameliorate microgliosis $[12,13]$. Although the studies referenced above suggested that VPA therapy is involved in microglial activation, those investigations determined activated microglia based only on cellular density in brain sections immunolabeled to detect either ionized calcium binding adaptor molecule 1 (Iba1) or CD11b [14]. There have been no studies, however, aimed at better characterizing the morphological changes and polarized phenotype of peri-infarct microglia after VPA administration. Meanwhile, VPA therapy has been demonstrated to cause dose-dependent side effects, like depression, ataxia, arrhythmia, and rare but fatal hepatotoxicity $[15,16]$. Relatively high doses of VPA of $300 \mathrm{mg} / \mathrm{kg} /$ day and above were shown to induce hepatotoxicity and neurological side effects in animal studies [17]. Thus, we aimed to study whether the post-stroke administration of a lower dose of VPA, $200 \mathrm{mg} / \mathrm{kg}$, in rats would promote recovery and whether this recovery, if any, is associated with altered biomarkers, cytokines, and transcriptional profiles within peri-infarct cortex. Furthermore, we quantitated the subtleties of microglial morphology with fractal analysis and circularity index following focal cortical ischemia-reperfusion injury, since morphology has not yet been well studied. As microglia/macrophages show obviously morphological changes on day 2 post-stroke in the ischemic cortex, we administered VPA after the onset of reperfusion. Here, we show how post-stroke of VPA 
administration, $200 \mathrm{mg} / \mathrm{kg}$, promoted behavioral recovery during the short 2-day testing period, accompanied by modulating microglia/macrophage activation, and further explore the underlying molecular mechanisms.

\section{Materials And Methods}

\section{Animals}

Male adult Sprague-Dawley rats $(300-350 \mathrm{~g})$ were purchased from the National Laboratory Animal Center, Taipei, Taiwan, R.O.C. All of the rats were housed in an environment with a 12-hour light/dark cycle, temperature of $25 \pm 2{ }^{\circ} \mathrm{C}, 55 \%$ humidity, 2-3 animals per cage, and ad libitum standard diet and water at the National Defense Medical Center's Animal Center, which is accredited by the Association for Assessment and Accreditation of Laboratory Animal Care International (AAALAC International). Their care was in accordance with institutional and international standards (Principles of Laboratory Animal Care, $\mathrm{NIH}$ ). The experimental protocol was approved by the Institutional Animal Care and Use Committee (IACUC; protocol number 16-258) of the National Defense Medical Center, Taiwan, R.O.C. All experiments were performed in a blinded manner, and the experimental results are reported according to the ARRIVE guidelines. The sample sizes for the experiments were estimated based on previous experience involving similar experimental settings.

\section{Distal middle cerebral artery occlusion model and VPA treatment}

A cortical stroke was induced in each rat by occluding the distal middle cerebral artery (dMCA) along with a bilateral common carotid artery (CCA) [18]. Each rat was anesthetized with $4 \%$ chloral hydrate (Sigma Aldrich, St. Louis, MO) injected intraperitoneally (i.p.; $400 \mathrm{mg} / \mathrm{kg}$ ), and lidocaine was used as a local anesthetic. The surgery was performed as described earlier [19-21]. Briefly, the CCAs were isolated through a cervical incision. A small craniotomy was made on the right side of the skull, and the right dMCA was ligated directly with a 10-0 suture. The CCAs were simultaneously occluded with nontraumatic arterial clips. After 90 minutes of ischemia, the suture around the MCA and the arterial clips were removed for restoration of blood flow (reperfusion). The body temperature of each rat was maintained at $37^{\circ} \mathrm{C}$ during the procedures until recovery from anesthesia, when the rat was returned to its home cage. A dose of $200 \mathrm{mg} / \mathrm{kg}$ of VPA or normal saline as a vehicle control was injected intraperitoneally into each rat, with the one injection made immediately after the onset of reperfusion. VPA was purchased from Sigma (MO, USA) and dissolved in physiologic saline. At day 2 post-stroke, the infarction volume was quantified with 2,3,5-triphenyltetrazolium chloride (TTC) staining from 2-mm brain slices as described previously [21].

\section{Behavioral tests}


A body asymmetry test, modified Bederson's neurological test, cylinder test, and analysis of locomotor activity were performed as described previously [21]. Briefly, the body asymmetry was analyzed from 20 consecutive trials by lifting the rats above the testing table by the tails and counting the frequency of initial turnings of the head or upper body contralateral to the ischemic side (the maximum impairment in stroke animals is 20 contralateral turns, whereas naïve animals turn in each direction with equal frequency resulting in 10 contralateral turns). Using the modified Bederson's score, the neurological deficits were scored according to the following criteria: $0=$ no observable deficit; 1 point $=$ rat showed decreased resistance to lateral push; 2 points $=$ rat kept the contralateral forelimb to the breast and extended the other forelimb straight when lifted by the tail, in addition to exhibiting the behavior indicated for score $1 ; 3$ points = rat twisted the upper half of its body towards the contralateral side when lifted by the tail, in addition to exhibiting the behaviors indicated for scores 1 and 2. The cylinder test was carried out as described previously [22]. In the cylinder test, the animal was placed inside a transparent vertical tube with a diameter of $35 \mathrm{~cm}$, and its movement was video recorded for 5 minutes. The number of first front paw touches to the inner wall of the tube, after raising back on the hind limbs, was counted. Locomotor activity was measured using an infrared activity monitor for one hour (Med Associates, St. Albans, VT).

\section{Assessment of cytokines}

Tissues from a total of 16 rats were used for the cytokine assay by enzyme-linked immunosorbent assay (ELISA). The ipsilateral peri-infarct cortical and hippocampal tissues were collected at 6, 24, and 48 hours post-dMCAo. After homogenization in lysis buffer (PRO-PREP ${ }^{\mathrm{TM}}$, iNtRON Biotechnology, Korea) and centrifugation at $12,000 \mathrm{~g}$ for 30 minutes, the supernatants were collected and stored at $-80^{\circ} \mathrm{C}$. During quantification, the cytokines (TNFa, IL-1 $\beta, \mathrm{IL}-6$, and IL-10) were normalized to $100 \mu \mathrm{g}$ of protein in the supernatant using a commercial ELISA kit from R \& D Systems (Minneapolis, MN, USA) according to the manufacturer's instructions.

\section{Histology, immunostaining of free-floating sections, and image acquisition}

The rats were deeply anaesthetized with pentobarbital ( $90 \mathrm{mg} / \mathrm{kg}$ i.p.) and transcardially perfused with $200 \mathrm{ml}$ saline followed by $500 \mathrm{ml}$ of $4 \%$ paraformaldehyde. Brains were processed for either paraffin or free-floating sections and stained with anti-CD11B antibodies. Brains were dehydrated in $30 \%$ sucrose at $4^{\circ} \mathrm{C}$ and sectioned coronally into $40-\mu \mathrm{m}$-thick slices using a Leica CM3050 Cryostat. Sections were taken from 2.1 to $-1.0 \mathrm{~mm}$ (striatum) relative to bregma, then stored in 1X PBS for short-term storage or in cryopreservant for long-term storage (20\% glycerol, $2 \%$ DMSO in 1XPBS). Sections were blocked with $0.3 \%$ hydrogen peroxidase and 4\% BSA (Sigma-Aldrich) $+0.3 \%$ Triton X-100 (Sigma-Aldrich), then incubated with primary antibody (rabbit anti-CD11B 1:1000; Abcam) overnight at $4^{\circ} \mathrm{C}$. The next day, sections were incubated with secondary antibody, followed by incubation with avidin-biotin complex and 
DAB as above. Digital imagining was carried out on an Olympus AX-80 microscope and attached DP-70 digital camera (Olympus America Inc., Center Valley PA) using a 40x objective. Three coronal brain sections per animal (between bregma -0.45 and -1.85 ) were imaged in the right hemisphere at each time point (sham, 1, 2, and 7 days post-dMCAo). The regions imaged were in the medial peri-infarct cortex extending $400 \mu \mathrm{m}$ from the infarct border and in the dorsal striatum underlying the infarct. Therefore, the imaging yielded six digital photomicrographs per animal for analysis.

\section{Unbiased stereological counting of CD11b-positive cells in the peri-infarct cortex}

CD11b-positive cells in the peri-infarct cortex were counted from 40- $\mu$ m-thick free-floating sections using unbiased stereology with a stereomicroscope (Olympus BX51) and the Stereolnvestigator 6 program (MBF Bioscience) as previously described [23]. The optical fractionator method, which involves utilizing a three-dimensional probe to count the cells of interest by randomly and systematically sampling each section, was used. It is unbiased, since it does not involve cell size and shape, and is unaffected by tissue processing. In this approach, the cell population is computed from the volume fraction. The volume fraction consists of information on the thickness of the tissue sampled, the number of sections sampled, and the area in each section sampled. Three $40-\mu \mathrm{m}$-thick coronal slices were selected based on their location relative to bregma $(0.2,-0.26$, and $-0.4 /-0.5 \mathrm{~mm})$ to obtain results relatively free of bias in the distribution of the cells. Only CD11b-positive cells with clear microglia morphology were counted. Approximately 60-80 randomly selected sites per area/slice were analyzed to ensure accuracy and minimize error.

\section{Fractal analysis using FracLac for ImageJ}

We extended our computer-aided morphologic analysis after cortical infarction to include fractal analysis (FracLac for ImageJ), which quantifies cell complexity (fractal dimension, DB) [24]. Four microglia were randomly chosen for fractal analysis (using a grid and random number generator) within each photomicrograph ( 6 photomicrographs per animal), for a total of 24 cells analyzed per animal in each region. The additional structures that abut and surround each cell were excluded from the analysis by manual deletion using a digitizing tablet and ImageJ. Binary images were then converted to outlines using ImageJ. Fractal dimension is a measure of microglia complexity, which quantifies each cell's contour bounded by the endpoints and process lengths. FracLac for ImageJ calculates the microglia fractal dimension (DB) for each cell using a box plot protocol that determines the amount of pixel detail with increasing scale, where $N=$ the number of pixels or "detail" at a particular scale ( $\nabla)$ (Fig. 6A; Table 1). These calculations and relationships are best summarized in the reference guide provided for FracLac for ImageJ https://imagej.nih.gov/ij/plugins/fraclac/FLHelp/Introduction.htm and additional associated references $[24,25]$. 


\section{Manual analysis of microglia morphology}

Manual morphological analysis was performed using FIJI software (Version 2.0) [26, 27]. Only slices containing an identified cell were processed as a maximum intensity projection to avoid overlapping with cells located in more superficial or deeper layers. Maximum intensity projections of the cells were thresholded to create a binary mask. Through measurement of the area (white) and the perimeter (red) of a cell from the binary mask, the circularity index $(\mathrm{Cl})$ was calculated (Fig. 6C, Table 1).

\section{Cell culture and treatment}

Murine BV-2 microglial cells were obtained from Dr. Mei-Jen Wang [28] and maintained in Dulbecco's modified Eagle's medium (DMEM) containing 5\% fetal bovine serum (FBS), $2 \mathrm{mM} \mathrm{L-glutamine,} 100 \mu \mathrm{g} / \mathrm{ml}$ streptomycin, and $100 \mathrm{U} / \mathrm{ml}$ penicillin (all from Gibco; Thermo Fisher Scientific, Inc., Waltham, MA, USA) at $37^{\circ} \mathrm{C}$ under humidified $95 \% 02$ and $5 \% \mathrm{CO} 2$. The cells were then seeded into 24 -well plates at a density of $1 \times 10^{5} /$ well and maintained at $37^{\circ} \mathrm{C}$ under humidified $95 \% 02$ and $5 \% \mathrm{CO} 2$. To detect the expression of acetyl-histone $\mathrm{H} 3$, as well as the production of galectin-3 and heat shock protein 70 (HSP70), the medium was removed and replaced with freshly prepared serum-free medium with or without lipopolysaccharide (LPS) $(1 \mu \mathrm{g} / \mathrm{ml})$ and/or a non-toxic dose of VPA (1.5 mM) for 6 hours [9].

\section{Western blot analysis}

For western blotting, aliquots of the proteins were separated by electrophoresis on sodium dodecyl sulfate-polyacrylamide gels (10\%) and transferred to a polyvinylidene difluoride membrane. Acetylated histone $\mathrm{H} 3$ was detected by using an antibody raised against acetylated histone $\mathrm{H} 3$ on lys 9 (rabbit polyclonal; 1:1000; sigma-Aldrich), HSP70 (rabbit polyclonal; 1:1000; Santa Cruz), and galectin-3 (rabbit polyclonal; 1:250; GeneTex). Briefly, the membranes were rinsed in $0.01 \mathrm{M}$ Tris-buffered saline (pH 7.4) containing $0.1 \%$ Triton X-100 for 30 minutes, blocked in $5 \%$ non-fat dry milk for 30 minutes, and then incubated overnight at $4^{\circ} \mathrm{C}$ with the primary antibody in Tris-buffered saline containing $3 \%$ non-fat dry milk. The membranes were then washed three times with Tris-buffered saline and incubated overnight at $4^{\circ} \mathrm{C}$ with a horseradish peroxidase-conjugated secondary antibody in Tris-buffered saline containing $3 \%$ non-fat dry milk. Immunoreactivity was detected by enhanced chemiluminescent autoradiography (ECL kit; Amersham Life Science, Arlington Heights, IL, USA) in accordance with the manufacturer's instructions. The western blots were captured with a digital camera, and the intensities were quantified with NIH Image J.

\section{Protein array analysis}

Brain protein levels were analyzed following dMCAo-induced cortical infarction. Rat brains were collected and homogenized 48 hours after dMACo, and the expression levels of 67 proteins were measured using a 
RayBio ${ }^{\circledR}$ L-Series Rat 67 Antibody Array (RayBiotech, Norcross, GA, USA). The whole brain was isolated from five rats in each group. Total protein was extracted from $250 \mathrm{mg}$ of brain tissue with $1 \mathrm{~mL}$ of icecold tissue protein extraction reagent containing protein degradation inhibitors (Kangcheng, Shanghai, China). Protein concentrations were determined using a BCA Protein Assay Kit (Kangcheng). The RayBio® L-Series Rat 67 Antibody Array membrane (RayBiotech) was blocked for 30 minutes by adding blocking buffer, and then incubated with the protein samples at room temperature for 1 to 2 hours. The samples were then discarded, the chip membrane was cleaned with buffer, and the membrane was then incubated with biotin-labeled antibodies at room temperature for 1 to 2 hours. The membrane was then washed with buffer and incubated with streptavidin (1:1000) coupled with horseradish peroxidase at room temperature for 2 hours. The membrane was thoroughly cleaned and then reacted with chemiluminescence reagent (RayBiotech, Norcross, GA, USA) in the dark and exposed to X-ray film, and images were obtained using a film scanner (i3200, Kodak, Rochester, NY, USA). The original biomarker values were first centered and scaled by subtracting the mean of each biomarker from the data and then dividing it by the standard deviation, respectively [29]. Centering and scaling results in a uniform mean and scale across all the biomarkers, but leaves their distribution unchanged [30, 31].

\section{Analysis of differential biomarker expression}

The biomarker values were summarized in terms of mean and standard deviation, or median with minimum and maximum responses across the groups. The fold change between groups was calculated as the ratio of the mean or median. If the biomarkers met or did not meet normality criteria across two groups, the significance of expression difference was evaluated by the paired t-test or signed-rank test, respectively. Biomarkers with a $\mathrm{P}$ adjusted value $<0.05$ were considered to be differentially expressed [30].

\section{RNA preparation and RNA-sequencing}

Rats were sacrificed on day 2 after MCAo surgery and perfused with $0.9 \%$ saline solution before the collection of tissue material ( $n=4$ for vehicle, $n=4$ for VPA). RNA was extracted from punch-samples of tissue that were taken from the medial peri-infarct cortex on two $1 \mathrm{~mm}$ thick sections from positions $A / P$ -0.9 to +0.1 and $A / P+1.1$ to +2.1 . RNA was extracted with Trizol reagent and treated with DNase (\#1906, Ambion). RNA purity and quantification were checked using SimpliNano ${ }^{\mathrm{TM}}$ - Biochrom

Spectrophotometers (Biochrom, MA, USA). RNA degradation and integrity were monitored by Qsep 100 DNA/RNA Analyzer (BiOptic Inc., Taiwan). A total amount of $1 \mu \mathrm{l}$ RNA per sample was used as input material for the RNA sample preparations. Sequencing libraries were generated using the KAPA mRNA HyperPrep Kit (KAPA Biosystems, Roche, Basel, Switzerland) following the manufacturer's recommendations, and index codes were added to attribute sequences to each sample. Briefly, mRNA was purified from total RNA using magnetic oligo-dT beads. Captured mRNA was fragmented by incubating it at a high temperature in the presence of magnesium in KAPA Fragment, Prime and Elute Buffer (1x). First-strand complementary DNA (cDNA) was synthesized using random hexamer priming. 
Combined second-strand synthesis and A-tailing, which converts the cDNA:RNA hybrid into doublestranded cDNA (dscDNA), was used to incorporate dUTP into the second cDNA strand, and then dAMP was added to the 3 ' ends of the resulting dscDNA. dsDNA adapters with 3'dTMP overhangs were ligated to library insert fragments to generate the library fragments carrying the adapters. In order to select cDNA fragments of preferentially 300 400bp in length, the library fragments were purified with the KAPA Pure Beads system (KAPA Biosystems, Roche, Basel, Switzerland). The library carrying appropriate adapter sequences at both ends was amplified using KAPA HiFi HotStart ReadyMix (KAPA Biosystems, Roche, Basel, Switzerland) along with library amplification primers. The strand marked with dUTP was not amplified, allowing strand-specific sequencing. Lastly, PCR products were purified using the KAPA Pure Beads system, and the library quality was assessed using the Qsep 100 DNA/RNA Analyzer (BiOptic Inc., Taiwan). The RNA-seq data have been deposited in the RNA-Seq database at Biotools-rat-RNA prelibrary under accession number BI-ANA-1416.

\section{Bioinformatics}

The original data obtained by high-throughput sequencing (with an Illumina NovaSeq 6000 platform) were transformed into raw sequenced reads by CASAVA base calling and stored in FASTQ format. FastQC and MultiQC [32] were used to check the fastq files for quality. The obtained raw paired-end reads were filtered by Trimmomatic (v0.38) [33] to discard low-quality reads, trim adaptor sequences, and eliminate poor-quality bases with the following parameters: LEADING:3 TRAILING:3 SLIDINGWINDOW:4:15 MINLEN:30. The obtained high-quality data (clean reads) were used for subsequent analysis. Read pairs from each sample were aligned to the reference genome (e.g., H. sapiens, GRCh38) by the HISAT2 software (v2.1.0)[34, 35]. FeatureCounts (v1.6.0) was used to count the reads numbers mapped to individual genes [36]. For gene expression, the "Trimmed Mean of M-values" normalization (TMM) was performed using DEGseq (v1.36.1) [37] without biological duplicate, and the "Relative Log Expression" normalization (RLE) was performed using DESeq2 (v1.22.1) [38, 39] with biological duplicate. Differentially expressed genes (DEGs) analysis of two conditions was performed in R using DEGseq (without biological replicate) and DESeq2 (with biological replicate), which are based on the negative binomial distribution and Poisson distribution model, respectively [40-42]. The resulting p-values were adjusted using Benjamini and Hochberg's approach for controlling the FDR. GO $[43,44]$ enrichment analysis of DEGs was conducted using clusterProfiler (v3.10.1) [31]. Gene set enrichment analysis (GSEA) [45] was performed with 1,000 permutations to identify enriched biological functions and activated pathways from the molecular signatures database (MSigDB). The MSigDB is a collection of annotated gene sets for use with GSEA software, including hallmark gene sets, positional gene sets, curated gene sets, motif gene sets, computational gene sets, GO gene sets, oncogenic gene sets, and immunologic gene sets $[46,47]$. In addition, weighted gene co-expression network analysis (WGCNA) was used to construct the co-expression network based on the correlation coefficient of expression pattern using the WGCNA (v1.64) package in R $[48,49]$. 


\section{Real-time quantitative PCR from microglia/macrophages isolated from the sham or stroke cortex}

The microglia harvest was performed as described previously [5]. Briefly, the CD11b immunopositive cortical microglia/macrophages were isolated by magnetic activated cell sorting (MACS). The ischemic cortex and the equivalent part of the brains from the sham animals were dissected on ice in HBSS without $\mathrm{Ca}^{2+}$ and $\mathrm{Mg}^{2+}$. The tissue was dissociated using the Neural Tissue Dissociation kit (Miltenyi Biotec) and gentleMACS Dissociator (Miltenyi Biotec). After dissociation, the cells were suspended in 0.5\% BSA in PBS and incubated with Myelin Removal Beads II (1:10, Miltenyi Biotec) for 15 minutes at $4^{\circ} \mathrm{C}$. The cells were washed and resuspended in 0.5\% BSA in PBS and filtered through an LS column (Miltenyi Biotec) using a Quadro-MACS Separator (Miltenyi Biotec). The total effluent was collected and resuspended in $0.5 \%$ BSA with $2 \mathrm{mM}$ EDTA in PBS. The cells were incubated at $4^{\circ} \mathrm{C}$ for 10 minutes with mouse anti-CD11b: FITC antibody (1:10, \#MCA275FA, AbD Serotec). The cells were washed, resuspended in $0.5 \%$ BSA with 2 mM EDTA in PBS, and incubated with anti-FITC MicroBeads (1:10, Miltenyi Biotec) for 15 minutes at $4^{\circ} \mathrm{C}$. The cells were washed and resuspended in $0.5 \%$ BSA with $2 \mathrm{mM}$ EDTA in PBS. The cell suspension was applied to a LS column placed on a QuadroMACS Separator. Total RNA was then extracted from the gentleMACS-isolated microglia/macrophage using RNeasy Plus micro/mini kits (Qiagen, Hilden, Germany), according to the manufacturer's protocol.

RNA quality was assessed using an Agilent Bioanalyzer (Agilent, Santa Clara, CA). For the rat samples, CDNA synthesis was carried out using an oligo-T18 primer and Maxima $\mathrm{H}$ minus reverse transcriptase (\#EP0751; Thermo Fisher Scientific). Real-time quantitative PCR (qPCR) was performed with a Lightcycler®480 real-time PCR system (Roche Diagnostics) using Lightcycler®480 SYBR Green I Master complemented with $2.5 \mathrm{pmol}$ of primers (Table 3 ) and cDNA corresponding to $10 \mathrm{ng}$ of total RNA in the final volume of $10 \mu \mathrm{l}$ on 384-well plates. Reactions were performed in duplicate or triplicate and analyzed with Lightcycler®480 Software. The length of the resulting PCR products was verified using agarose gel electrophoresis. Gene expression was normalized to the geometric mean of ubiquitin-conjugating enzyme E2 I (Ube2i) expression levels.

\section{Statistical analysis}

All graphs and statistics were performed in GraphPad Prism 6.0. The body asymmetry, Bederson's score, and locomotor activity data were analyzed using Bonferroni's post hoc test following two-way ANOVA. The infarction volume and qPCR results were analyzed using the two-tailed Student's $t$-test. Two-way ANOVA and Tukey's multiple comparisons post hoc test with the factors of "time" and "drug treatment" were used for the comparison of differences in ELISA results and parameters quantified from immunohistochemical staining. Values are presented as mean \pm S.E.M. A statistically significant difference was defined as $p<0.05$. 


\section{Results}

\section{Low-dose VPA treatment does not reduce infarction volume, but promotes short-term behavioral recovery in rat cortical stroke model}

In contrast with the results reported by prior studies, a single injection of the dose of VPA $(200 \mathrm{mg} / \mathrm{kg})$ had no effect on infarct volume measured at 48 hours after stroke (Fig. 1A-C). However, the treatment produced a rapid recovery of function, as seen from significantly reduced body asymmetry and Bederson's scores at 24 hours post-stroke (Fig. 1D, E). At 48 hours, the rats injected with VPA also exhibited substantial reversal of injury-induced behavioral deficits in terms of body asymmetry, Bederson's neurological deficits, and the cylinder tests compared to the control group of vehicle-injected rats (Fig. 1D-F). Spontaneous motor activity, as another indicator of hastened recovery, did not differ between the treatment groups (Fig. 1G, H). These results clearly indicate that the low-dose VPA treatment significantly promoted functional recovery without reducing the size of the ischemic lesions.

\section{VPA decreases the production of IL-6, but not TNF-a or IL- $1 \beta$ in the peri-infarct cortex}

Since the levels of pro-inflammatory cytokines in experimental stroke have significant effects on infarction evolution [4,50], we first characterized the cytokine responses in the proximal (peri-infarct cortex) and distal (ipsilateral hippocampus) region after dMCAo-induced cortical stroke, and then tested the efficacy of post-stroke subcutaneous VPA treatment in adult rats. Obvious increases in IL-1 $\beta$, TNF-a, and IL- 6 were observed as soon as 6 hours after IMCAo within the peri-infarct cortex, where they remained elevated for 2 days after dMCAo (Fig. 2A, C, E). Of additional interest, down-regulated IL-10 was observed 6 hours after dMCAo in the peri-infarct cortex, where it remained decreased until 24 hours (Fig. 2G). In the area distal from infarct cortex (hippocampus), there were no differences in the levels of IL-1 $\beta$, TNF- $\alpha$, and IL-10 between the sham and dMCAo groups at different time points (Fig. 2B, F). However, increased IL- 6 was observed in hippocampal brain homogenates 6 hours after dMCAo, where it remained elevated until 2 days (Fig. 2D). These results suggest that dMCAo-induced up-regulation of IL-6 is not only involved in the region proximal to the infarct cortex, but also in the region distal to it (hippocampus).

To determine whether post-stroke VPA treatment is involved in inflammatory responses, we measured changes in the levels of pro- and anti-inflammatory cytokines in the peri-infarct cortex and hippocampal brain homogenates at 6,24 , and 48 hours after dMCAo. The administration of VPA resulted in a significant decrease in IL-6 within the peri-infarct cortex at 24 hours after dMCAo (Fig. 2C), but not in the ipsilateral hippocampal area (Fig. 2D). Meanwhile, the levels of IL-1, TNF-a, and IL-10 within the periinfarct cortex and ipsilateral hippocampus exhibited no statistically significant differences between the 


\section{Post-insult VPA administration reduces the number of CD11b-positive cells on day 7}

VPA has been demonstrated to exhibit a neuroprotective property in a rat model of dMCAo by promoting neuronal survival as well as exerting anti-inflammatory effects, which would contribute to a reduction in microglial cell numbers [50]. However, how post-stroke VPA treatment modulates the time course of microglia/macrophage activation in the peri-infarct area has not yet been investigated. The infarct was already well advanced at 6 hours after dMCAo, reached a maximum volume by 48 hours, and had contracted greatly by 7 days based on the volume of tissue exhibiting pale staining with TTC (Fig. 3A, C, E). Thus, we first characterized the time course of CD11b-positive microglia/macrophages in the dMCAoinduced ischemic cortex. At 6 hours post-stroke, few CD11b-positive microglia/macrophages with ramification were present in the peri-infarct region (Fig. 3B). Microglia/macrophage activation peaked on day 2 post-stroke in the ischemic cortex, when the infarct core and peri-infarct zone were filled with CD11 $\mathrm{b}^{+}$cells presenting with an amoeboid shape (Fig. 3D). At 7 days post-stroke, CD11b-positive cells were obvious evident in the peri-infarct zone, but the morphology of microglia/macrophages with ramification of cell processes seemed to have recovered partially (Fig. 3E). VPA treatment did not obviously reduce the number of CD11b-positive microglia/macrophages on day 2 post-stroke (Fig. 4C, D, G), but significantly decreased the CD11b-positive cell number in the peri-infarct zone at day 7 post-stroke (Fig. 4E-G). These results suggested that the low-dose VPA treatment has delayed effects on the recruitment of microglia/macrophages into ischemic cortex.

\section{VPA treatment regulates morphologic responses of CD11b- positive cells after ischemic brain injury}

To further characterize the morphological responses of the microglia/macrophages, the fractal dimensions and cell circularity of CD11b-immunolabeled cells were measured in separate sections from the same rats at 2 days and 7 days post-stroke. Examples of CD11b-positive microglia/macrophages (made binary and outlined) in the peri-infarct cortex with/without VPA administration are shown in Fig. 5. The application of FracLac for Image $\mathrm{J}$ to microglia outlines resulted in fractal dimensions that ranged from 1.0029 to 1.4058 (available range is $1-2$ ), with the lowest occurring in the peri-infarction region at day 2 post-stroke and the highest in the intact cortex with sham surgery (Fig. 5B, D; 6B). There was no difference in the fractal dimensions of CD11b-immunolabeled cells in the intact cortex with or without VPA administration (Fig. 5C; 6B). However, two-way ANOVA analysis showed that VPA treatment could increase the fractal dimensions of CD11b-labelled microglia/macrophages at 2 days and 7 days poststroke, compared with the saline group (Fig. 5D-G; 6B). The above results suggest that microglial complexity is unchanged from sham in the impact region with or without VPA application, whereas, in the 
peri-infarct region, cell complexity was lower at 2 days and 7 days post-stroke and was recovered close to the sham condition through VPA therapy. Using manual analysis, we investigated an additional measure of microglial morphology related to cell circularity: the circularity index. The circularity index results of the CD11b-labelled cells in the sham group was similar to that of the CD11b-labelled cells in the VPA-treated group (Fig. 6D). However, the circularity index was maximally increased at 48 hours post-stroke, and remained similarly elevated by 7 days (Fig. 6D). The administration of VPA caused a significant reduction in the circularity index of CD11b-positive cells compared with the vehicle-treated group when assessed at 2 days and 7 days after stroke (Fig. 6D). Based on qualitative observations of the CD11b-labeled cell complexity and circularity, it was suggested that VPA administration could be implicated in microglia/macrophage activation in the peri-infarct region.

\section{VPA suppresses the level of galectin-3 in the peri-infarct cortex and LPS-treated microglia}

It is widely accepted that epigenetic processes occur during ischemic stroke [51]. HDAC inhibitors are known to promote a phenotypic shift in microglia and subsequently exert neuroprotective effects in animal models of neurodegenerative diseases $[52,53]$. VPA has been shown to exhibit anti-inflammatory properties by suppressing the number of activated microglia in stroke rats [50]. However, it is much less clear whether VPA ameliorates the activation of microglia/macrophages through the regulation of HDACs or the modulation of certain protein expression levels in these cells. Therefore, we adopted the antibody array analysis of 67 proteins in sham-operated, vehicle-treated, and VPA-treated ipsilateral cortex 2 days after dMCAo (Table. 2). Only proteins with fold change $>2$ or $<0.25$ and which were significantly different (adjusted $\mathrm{P}<0.05$ ) were included (Fig. 7A). The level of galectin-3 was significantly higher in the vehicletreated stroke rats compared with the sham-operated rats (Fig. 7B). However, VPA treatment significantly suppressed the level of galetin-3 in the ischemic cortex compared with the vehicle-treated group cortex (Fig. 7B). Western blotting showed that the galectin-3 protein level was remarkably reduced in the VPAtreated peri-infarct cortex (Fig. 7C, D), consistent with the results obtained with the protein array analysis. In previous studies, the VPA dose used in animal studies to control seizures, $300 \mathrm{mg} / \mathrm{kg}$, was demonstrated to induce an increase in acetylated histone $\mathrm{H} 3$ levels and to up-regulate HSP70 levels in the brain. In our study, the dosage of VPA, revised down to $200 \mathrm{mg} / \mathrm{kg}$, also increased acetylated histone H3 levels in the peri-infarct cortex compared with vehicle-treated group cortex (Fig. 7C, D). However, there was no difference in the levels of HSP70 between the two groups (Fig. 7C, D). Since galectin-3 is expressed mainly in microglia/macrophages and up-regulated when activated, we next determined the epigenetic mechanism whereby VPA may regulate galectin-3 production in these cells. BV2 cells were challenged with $1 \mu \mathrm{g} / \mathrm{ml}$ LPS in the presence or absence of $1.6 \mathrm{Mm}$ VPA for 6 hours. Acetylated histone H3, galectin3 , and HSP70 expressions in the BV2 cells were subsequently assessed. As presented in Fig. 7E, the addition of VPA resulted in an increase over the baseline acetylated histone H3 level but decreased galectin-3 expression compared with untreated control. In addition, when cells were treated with LPS in the presence of VPA, acetylated histone $\mathrm{H} 3$ levels were significantly increased when compared with those 
receiving LPS treatment alone (Fig. 7F). In contrast, administration with VPA was found to significantly decrease galectin-3 expression in LPS-treated cells as compared to LPS treatment alone (Fig. 7F). However, the treatment of VPA had no effect on HSP70 expression under the condition of exposing BV-2 cells with or without LPS (Fig. 7E, F). These results suggest that VPA, by promoting histone acetylation on $\mathrm{H} 3$, inhibits galectin-3 production in the microglia/macrophages under the baseline condition or in response to injurious stimuli.

\section{Differential gene expression in peri-infarct cortex with or without VPA treatment}

Since the low dose of VPA was implicated as having an effect on the level of galectin-3 and in the morphological changes of microglia in the peri-infarct cortex, we naturally hypothesized that VPA treatment would regulate genes involved in tissue repair, as well as the phenotypes of immune cells. Comparison of gene profiles between vehicle-treated and VPA-treated groups was focused on the periinfarct cortex at 3 days post-stroke (Fig. 8A). Genes were identified as differentially expressed genes (DEGs) only when the fold difference between two groups was greater than 2 and the adjusted $p$ values were lower than or equal to 0.05 . Volcano plots that graphically highlighted the DEGs that were significantly up- (red) or down- (blue) regulated in response to VPA treatment and vehicle treatment in the stroke rats were generated (Fig. 8B). In the VPA-treated group, 7 transcripts were differentially expressed compared to the vehicle-treated group, among which 1 gene was downregulated and 6 genes were upregulated (Fig. 8C). The one down-regulated gene was a pseudogene. The up-regulated genes were related to components of the extracellular matrix (collagen 3a1, collagen 6a2, decorin, and cellular communication network factor 1 ) and included genes which code for coagulation-related surface proteins on the endothelial cells (thrombomodulin) and macrophage galactose-type lectin on M2 microglia (C-type lectin domain containing 10A) (Fig. 8C). These data revealed a prominent upregulation of gene expression related to extracellular matrix remodeling in the stroke rats that received VPA treatment.

\section{Gene ontology (GO) analysis from VPA- and vehicle-treated ischemic brains}

To further reveal the functional categories of DEGs, gene ontology (GO) enrichment analysis was performed. GO enrichment analysis of DEGs (dMCAo + vehicle vs. dMCAo + VPA) identifies key biological processes, cellular components, and molecular functions. The top 3 significantly upregulated functional categories (adjusted P value $<0.05$ ) were enriched by DEGs in the VPA-treated group, with those DEGs being involved in wound healing, collagen trimmer and extracellular matrix functions (Fig. 8D, E). Notably, the DEGs related to wound healing were also partially associated with collagen trimmer and extracellular matrix functions, suggesting that post-stroke VPA treatment might potentiate tissue remodeling in the peri-infarct cortex. Network-based GSEA (gene set enrichment analysis) was utilized to identify the 
functional associations amongst the genes affected by the VPA treatment (Fig. 8F). Similar to the results obtained using enriched $\mathrm{GO}$ analysis, the top 10 functions of the up-regulated genes were associated with the extracellular space (adjusted $P$ value $=0.044$ ), drug responses (adjusted $P$ value $=0.044$ ), extracellular matrix (adjusted $P$ value $=0.044$ ), positive regulation of gene expression (adjusted $P$ value $=0.044$ ), basement membrane (adjusted $P$ value $=0.044$ ), external side of the plasma membrane (adjusted $P$ value $=0.044$ ), cell-cell adhesion (adjusted $P$ value $=0.046$ ), wound healing (adjusted $P$ value $=0.046$ ), heparin binding (adjusted $P$ value $=0.046)$, and angiogenesis (adjusted $P$ value $=0.046$ ).

\section{Post-stroke VPA treatment regulates gene expression profile of activated microglia/macrophages}

Given that the C-type lectin domain containing 10A (Clec10a) was mainly expressed on the antigenpresenting cells and macrophages [54], we wondered whether the upregulated Clec10a in VPA-treated peri-infarct cortex was also on the activated microglia/macrophages. Therefore, we isolated CD11bpositive cells from the infarct area at day 3 post-stroke (Fig. 9A), and then analyzed the mRNA level of Clec10a in these cells. Similar to the results obtained from immunofluorescence, VPA treatment did not obviously reduce the number of CD11b-positive microglia/macrophages on day 3 post-stroke (Fig. 9B). However, a significant increase in the mRNA level of Clec10a in the CD11b-positive microglia/macrophages was shown in the VPA-treated group (Fig. 9C), suggesting that treatment with VPA increased Clec10a expression in the peri-infarct cortex and on activated microglia and macrophages. Since Clec10a has immunoregulatory properties and is induced on M2 microglia in neurological autoimmune disorders [55], we next wanted to explore whether VPA could regulate the phenotypes or gene expression profiles of microglia. For additional evaluations of the status of microglia/macrophages, the mRNA levels of selected markers of the cytotoxic M1 phenotype (iNOS and CD86) and the cytoprotective M2 phenotype (TGF $\beta 1, C D 163$, Arginase-1) were analyzed in these CD11 b cells. Our results indicated that VPA treatment does not change the expressions of $i N O S$ or CD86, genes expressed in cytotoxic M1-type microglia (Fig. 9C). In turn, the analysis of markers of M2 phenotype revealed a significant increase in the mRNA levels of TGFB1 and CD163 but not Arginase-1 in the CD11 ${ }^{+}$ microglia/macrophages in response to VPA exposure (Fig. 9C), implying that VPA treatment did not cause collective up-regulation of M2-type marker genes in the peri-infarct cortex. Taken together, these findings indicate that post-stroke VPA treatment not only stimulates extracellular matrix remodeling, but also alters the morphological responses and gene expressions of activated microglia/macrophages in the peri-infarct cortex.

\section{Discussion}

This study provides evidence that a low dose of VPA post-stroke can improve the recovery of aspects of neurological function in rats, and that this effect of VPA is associated with the suppression of peri-infarct activity in microglia/macrophages, including activity related to morphological changes and density. This 
attenuation of microglial activity may involve the suppression of galectin-3 expression and the upregulation of extracellular matrix remodeling in the peri-infarct cortex.

Accumulating evidence supports the notion that histone hypoacetylation and transcriptional dysfunction are involved in a large number of neurodegenerative conditions [56]. Although postischemic treatment with a clinically relevant dose of VPA, specifically, $300 \mathrm{mg} / \mathrm{kg}$, has been shown to reduce dMCAo-induced brain infraction, BBB disruption, and brain edema $[2,10]$, this dose is too high for humans. As with many antiepileptic drugs, there are a number of adverse consequences associated with the use of VPA. Among these, valproate encephalopathy is a dose-related syndrome that can occur due to high VPA concentrations, particularly in new patients [57]. Moreover, VPA-associated pancytopenia and coagulopathy have been shown to be dose-dependent side effects [58]. Furthermore, the teratogenicity of VPA limits its use in woman of childbearing age [59]. In this study, we reduced the dose to $200 \mathrm{mg} / \mathrm{kg}$ of VPA and tested the therapeutic effects of this dose in rats. The results indicated that a single injection of VPA at a dose of $200 \mathrm{mg} / \mathrm{kg}$ in stroke rats did not reduce infarct volume but did significantly accelerate the reversal of behavioral deficits in rats with cerebral ischemic injury. The improvements of forepaw function and body symmetry occurred in the absence of changes in infarct volume and are broadly consistent with reports of improved outcomes without changes in infarct volume following VPA treatment in several studies [60]. Thus, the findings of the present study suggest that cellular changes critical for recovery are initiated in the early stages after infract formation, and that they can be modulated by VPA treatment.

It has become increasingly that clear brain injury following ischemia is highly associated with the inflammatory response, which involves the infiltration of mononuclear phagocytes and activated microglia [61]. Inflammatory cytokines (IL-1 $\beta$, TNF-a, and IL-6) produced by peri-infarct

microglia/macrophages would modulate tissue injury and have profound effects on infarct evolution [4]. In the past literature, VPA was demonstrated to inhibit TNF- $a$ and IL- 6 production induced by LPS in THP1 cells, and this inhibition was linked to the suppression of NF-KB activation [9]. In animal studies of global brain ischemia, VPA further suppressed IL-1 $\beta$ production in the hippocampus [14]. Studies of cellular sources of TNF- $\alpha$ and IL-1 $\beta$ after MCAo have looked specifically at microglia and macrophages. However, IL- 6 is upregulated in microglia and cortical neurons in stroke rats, and increases of IL- 6 are more pronounced in the case of gray matter lesions $[62,63]$. Based on our results, a single injection of VPA at a dose of $200 \mathrm{mg} / \mathrm{kg}$ was shown to suppress the up-regulation of IL-6 in cortex at 24 hours following dMCAo, suggesting that low-dose VPA therapy might both alleviate neuronal damage and dampen microglial activation in the early phase of cerebral ischemic injury.

The single injection of a low dose of VPA after stroke did not produce significant differences in the number of CD11b-positive microglia/macrophages within the peri-infarct cortex within the first 2 days. Thus, our findings suggest that the low dose of VPA did not produce gross alterations in CD11b-positive cell recruitment to the peri-infarct tissue in the early phase. However, in other studies regarding the effect of VPA on microglia/macrophage distribution, VPA was shown to greatly limit microglial density in the injured area $[14,50]$. This difference in response to focal ischemia compared with that in the present 
study is probably explained, at least in part, because the higher dose of VPA in those other studies produced decreases in cell loss and infarct size in those earlier investigations of stroke, and such protective effects can secondarily reduce microglial responses to ischemic damage.

Apart from the changes in microglial distribution in response to ischemic damage, ramified microglia would transform to amoeboid morphology and resume phagocytotic activity, which exacerbate neuroinflammatory responses $[64,65]$. It follows that microglia morphology, if quantified using sensitive methods, could provide insight into the neuropathology of distinct regions. Using two quantitative assessments of microglia complexity and circularity, we report that the microglia morphology was different from that in sham cortex in the peri-infarct cortex at 2 and 7 days post-stroke. In the peri-infarct region, most of the microglia became amoeboid or had increased circularity, while ramification in shape or higher complexity was less observed. One striking finding of our results is that the low-dose VPA treatment produced significant differences in the complexity and circularity of CD11b-positive microglia within 7 days compared with vehicle treatment, indicating that VPA greatly limits changes in microglial morphology after dMCAo. Simultaneously, the low-dose VPA treatment was shown to not only enhance the levels of acetylated histone $\mathrm{H} 3$ protein but also to suppress the levels of galectin-3 in the peri-infarct cortex. Since galectin-3 is predominantly expressed in the microglia of the adult rodent brain $[66,67]$, we further argue that VPA treatment could suppress the LPS-upregulated galectin-3 levels in BV2 cells. It has been shown that galectin-3 could control microglia morphology by regulating the cytoskeleton $[67,68]$. Thus, amoeboid microglia are rich in galectin-3 and display productive phagocytosis. Moreover, galectin-3 is required for microglia-mediated brain inflammation $[69,70]$, while the suppression of galectin-3 ameliorates microglia-mediated pathogenesis by decreasing NFKB activation [70]. Interestingly, NFKB motifs have been identified in the promotor region of galectin-3 [71], implying that NFKB and galectin-3 might be regulated in a positive feed-forward loop in microglia. In our study, VPA was shown for the first time to promote a morphological shift of peri-infarct microglia from the amoeboid to the ramified form, and that might be associated with galectin-3 suppression in the peri-infarct cortex or microglia. Although previous studies have found some epigenetic pathways wherein the chromatin remodeling proteins contribute to galectin-3 induction [72], we have not yet examined whether VPA regulating the level of galectin-3 is directly dependent on the epigenetic action or upregulation of the acetylation of NFKB p65 caused by the decrease of HDAC3 activity [2].

An increasing number of studies have demonstrated that VPA treatment could directly up-regulate genes associated with neuronal proliferation, differentiation, and neurotransmission, while down-regulating genes related to cell death and inflammation [73]. Such changes may play roles in the benefits provided by VPA treatment following injury. In our study, RNA sequencing further revealed that VPA treatment exhibits a strong trend of upregulation of genes related to extracellular matrix remodeling in the periinfarct cortex. Collagen 3a1, collagen 6a2, decorin, and cellular communication network factor 1 , all of which are known to be part of the extracellular matrix, serve essential functions involved in the regulation of cellular processes and providing a permissive microenvironment to promote tissue repair [74-79]. Moreover, the provisional extracellular matrix serves as a pliable scaffold wherein mechanical guidance forces are established among endothelial cells, thereby providing critical support for vascular 
endothelium [76]. Thrombomodulin is a membrane protein mainly expressed by endothelial cells. It is part of the anticoagulant protein $\mathrm{C}$ system, in which thrombomodulin binds with thrombin and promotes the cleavage of protein $\mathrm{C}$ and thrombin activatable fibrinolysis inhibitor, thereby inhibiting coagulation [80]. Additionally, it interferes with inflammation, stabilizes barrier function, and promotes angiogenesis under pathological conditions [81]. Clec10a is a member of the C-type lectin receptor family and is expressed by myeloid antigen-presenting cells (APC), such as dendritic cells and macrophages [55]. Notably, Clec10a has also been shown to be upregulated on M2 microglia, which are involved in the processes of extracellular matrix reconstruction and tissue repair in the injured area. GSEA using GO also demonstrated a pattern of increased extracellular matrix, cell-cell adhesion, angiogenesis, and wound healing. Thus, the fact that these changes correlate with activated tissue repair and extracellular matrix suggests that VPA may help to restore normal functions.

In the present study, the analysis of VPA-induced changes to gene expression in the peri-infarct CD11bpositive cells at early time points revealed an up-regulation of the expression of Clec10a, TGF 1 , and CD163, but not Arginase-1. These genes are expressed in M2 phenotype microglia. The effect of VPA in terms of altering the expression of markers of alternative, anti-inflammatory microglia (M2 phenotype) is in agreement with what has been reported after spinal cord injury [13], but unlike what the authors of that report proposed, our data suggest that, during the early phase of cerebral ischemic injury, VPA did not decrease the expression of M1-associated markers (iNOS, CD86). This discrepancy implies that the single injection with a low dose of VPA only stimulated certain subtypes of alternatively activated microglia during pathology progression. Primarily M2 phenotype microglia with high expression of Arginase-1 contribute to axon regeneration through anti-inflammatory effects in neurodegenerative diseases [82]. CD163 is a phagocytic marker of microglia/macrophages that functions as a membrane-bound scavenger receptor for cleaning extracellular haptoglobin-hemoglobin and has an immunoregulatory property associated with the resolution phase of inflammation [83]. TGF $\beta 1$ has been recently shown to potentiate an adaptive activation of microglia to accelerate wound healing [84]. Collectively, these genes of the M2 phenotype are essential for repair processes, and their high expression in our study might reflect peri-infarct tissue repair in response to VPA treatment.

There were several limitations to the present study. First, although our data indicated that $200 \mathrm{mg} / \mathrm{kg}$ of VPA still exhibits a therapeutic effect in ischemic brain injury, we did not measure the kinetics of VPA in rats, and the dose we used on the rats in this study cannot be extrapolated to a human-equivalent dose. Second, our data demonstrated that post-stroke VPA treatment could suppress the upregulation of galectin-3, which is required for resident microglia activation in response to ischemic injury. However, galectin-3 knockout microglia failed to activate and proliferate, which was further associated with significant increases in the size of the ischemic lesions [66]. Thus, it is still necessary to elucidate the time courses of galectin-3 expression and function in microglia under pathological conditions. Finally, we did not have gene expression data from a sham group, so it is unclear if the effects of VPA on gene expression are restorative or de novo. Additional studies are already underway to fill in many of these gaps. 
In conclusion, we found that a single injection of VPA following dMCAo was able to accelerate functional recovery in rats. Both mitigating microglia/macrophage activation through the suppression of upregulated galectin-3 and altering the gene profiles of these cells could influence extracellular matrix reconstruction and contribute to the improved recovery.

\section{Abbreviations}

AAALAC International: Association for Assessment and Accreditation of Laboratory Animal Care International

APC: Antigen-presenting cells

BBB: Blood-brain barrier

CCA: Common carotid artery

cDNA: Complementary DNA;

Cl: Circularity index

DEGs: Differentially expressed genes

dMCA: Distal middle cerebral artery

dMCAo: Distal middle cerebral artery occlusion

DMEM: Dulbecco's modified Eagle's medium

dscDNA: Double-stranded cDNA

ELISAs: Enzyme-linked immunosorbent assays

FBS: Fetal bovine serum

GSEA: Gene set enrichment analysis

HDAC: Histone deacetylase

HSP70: Heat shock protein 70

IACUC: Institutional Animal Care and Use Committee

Iba1: Ionized calcium binding adaptor molecule 1

LPS: Lipopolysaccharide

MACS: Magnetic activated cell sorting 
qPCR: Quantitative PCR

RLE: Relative Log Expression

TMM: Trimmed Mean of M-values

TTC: 2,3,5-triphenyltetrazolium chloride

VPA: Valproic acid

WGCNA: Weighted gene co-expression network analysis.

\section{Declarations}

\section{Ethics approval and consent to participate}

The experimental protocol was approved by the Institutional Animal Care and Use Committee (IACUC; protocol number 16-258) of the National Defense Medical Center, Taiwan, R.O.C. All experiments were performed in a blinded manner, and the experimental results are reported according to the ARRIVE guidelines. The sample sizes for the experiments were estimated based on previous experience involving similar experimental settings.

\section{Consent for publication}

Not applicable

\section{Availability of data and materials}

The dataset(s) supporting the conclusions of this article is(are) included within the article (and its additional file(s)).

\section{Competing interests}

The authors declare there are no conflicts of interest.

\section{Funding}

Supported by The Ministry of Science and Technology of Taiwan, ROC, MOST-2314-B-016-001-MY3, MOST-108-2314-B-016-058-MY2, and MOST-108-2314-B-016-027; Medical Research Project grants TSGHC108-098, TSGH-C108-094, TSGH-D109-100, TSGH-D109-099 and TSGH-D109-097 from the Tri-Service General Hospital of Taiwan; the National Defense Medical Center, ROC, MAB-108-025. 


\section{Authors' contributions}

TT Kuo, YH Chen, TK Yin: conception and design; TT Kuo, V Wang, TK Yin: performing experiments, data analysis, manuscript writing; TT Kuo, JS Wu, YH Chen, TK Yin: planning experiments, data analysis, manuscript revision.

\section{Acknowledgements}

The authors thank Dr. Mei-Jen Wang for the murine BV-2 microglial cell support.

\section{References}

1. Feigin VL, Krishnamurthi RV, Parmar P, Norrving B, Mensah GA, Bennett DA, Barker-Collo S, Moran AE, Sacco RL, Truelsen T, et al: Update on the Global Burden of Ischemic and Hemorrhagic Stroke in 1990-2013: The GBD 2013 Study. Neuroepidemiology 2015, 45:161-176.

2. Wang Z, Leng Y, Tsai LK, Leeds P, Chuang DM: Valproic acid attenuates blood-brain barrier disruption in a rat model of transient focal cerebral ischemia: the roles of HDAC and MMP-9 inhibition. $J$ Cereb Blood Flow Metab 2011, 31:52-57.

3. Wang J, Wang J, Wang J, Yang B, Weng Q, He Q: Targeting Microglia and Macrophages: A Potential Treatment Strategy for Multiple Sclerosis. Front Pharmaco/ 2019, 10:286.

4. Lambertsen KL, Biber K, Finsen B: Inflammatory cytokines in experimental and human stroke. $J$ Cereb Blood Flow Metab 2012, 32:1677-1698.

5. Anttila JE, Albert K, Wires ES, Matlik K, Loram LC, Watkins LR, Rice KC, Wang Y, Harvey BK, Airavaara M: Post-stroke Intranasal (+)-Naloxone Delivery Reduces Microglial Activation and Improves Behavioral Recovery from Ischemic Injury. eNeuro 2018, 5.

6. Lakhan SE, Kirchgessner A, Hofer M: Inflammatory mechanisms in ischemic stroke: therapeutic approaches. J Transl Med 2009, 7:97.

7. Ahmad M, Graham SH: Inflammation after stroke: mechanisms and therapeutic approaches. Trans/ Stroke Res 2010, 1:74-84.

8. Chateauvieux S, Morceau F, Dicato M, Diederich M: Molecular and therapeutic potential and toxicity of valproic acid. J Biomed Biotechnol 2010, 2010.

9. Mairuae $\mathrm{N}$, Cheepsunthorn P: Valproic acid attenuates nitric oxide and interleukin-1beta production in lipopolysaccharide-stimulated iron-rich microglia. Biomed Rep 2018, 8:359-364.

10. Ren $M$, Leng $Y$, Jeong $M$, Leeds $P R$, Chuang $D M$ : Valproic acid reduces brain damage induced by transient focal cerebral ischemia in rats: potential roles of histone deacetylase inhibition and heat shock protein induction. J Neurochem 2004, 89:1358-1367.

11. Liu XS, Chopp M, Kassis H, Jia LF, Hozeska-Solgot A, Zhang RL, Chen C, Cui YS, Zhang ZG: Valproic acid increases white matter repair and neurogenesis after stroke. Neuroscience 2012, 220:313-321. 
12. Masuch $\mathrm{A}$, Shieh $\mathrm{CH}$, van Rooijen $\mathrm{N}$, van Calker D, Biber K: Mechanism of microglia neuroprotection: Involvement of P2X7, TNFalpha, and valproic acid. Glia 2016, 64:76-89.

13. Chen S, Ye J, Chen X, Shi J, Wu W, Lin W, Lin W, Li Y, Fu H, Li S: Valproic acid attenuates traumatic spinal cord injury-induced inflammation via STAT1 and NF-kappaB pathway dependent of HDAC3. $J$ Neuroinflammation 2018, 15:150.

14. Xuan A, Long D, Li J, Ji W, Hong L, Zhang M, Zhang W: Neuroprotective effects of valproic acid following transient global ischemia in rats. Life Sci 2012, 90:463-468.

15. Anderson GD, Acheampong AA, Wilensky AJ, Levy RH: Effect of valproate dose on formation of hepatotoxic metabolites. Epilepsia 1992, 33:736-742.

16. Isoherranen N, Yagen B, Spiegelstein O, Finnell RH, Merriweather M, Woodhead JH, Wlodarczyk B, White HS, Bialer M: Anticonvulsant activity, teratogenicity and pharmacokinetics of novel valproyltaurinamide derivatives in mice. $\mathrm{Br} J$ Pharmacol 2003, 139:755-764.

17. Tai YT, Lee WY, Lee FP, Lin TJ, Shih CL, Wang JY, Chiu WT, Hung KS: Low dose of valproate improves motor function after traumatic brain injury. Biomed Res Int 2014, 2014:980657.

18. Chen ST, Hsu CY, Hogan EL, Maricq H, Balentine JD: A model of focal ischemic stroke in the rat: reproducible extensive cortical infarction. Stroke 1986, 17:738-743.

19. Matlik K, Abo-Ramadan U, Harvey BK, Arumae U, Airavaara M: AAV-mediated targeting of gene expression to the peri-infarct region in rat cortical stroke model. $J$ Neurosci Methods 2014, 236:107113.

20. Airavaara M, Chiocco MJ, Howard DB, Zuchowski KL, Peranen J, Liu C, Fang S, Hoffer BJ, Wang Y, Harvey BK: Widespread cortical expression of MANF by AAV serotype 7: localization and protection against ischemic brain injury. Exp Neurol 2010, 225:104-113.

21. Airavaara M, Shen H, Kuo CC, Peranen J, Saarma M, Hoffer B, Wang Y: Mesencephalic astrocytederived neurotrophic factor reduces ischemic brain injury and promotes behavioral recovery in rats. $J$ Comp Neuro/ 2009, 515:116-124.

22. Runeberg-Roos P, Piccinini E, Penttinen AM, Matlik K, Heikkinen H, Kuure S, Bespalov MM, Peranen J, Garea-Rodriguez E, Fuchs E, et al: Developing therapeutically more efficient Neurturin variants for treatment of Parkinson's disease. Neurobiol Dis 2016, 96:335-345.

23. Mijatovic J, Airavaara M, Planken A, Auvinen P, Raasmaja A, Piepponen TP, Costantini F, Ahtee L, Saarma M: Constitutive Ret activity in knock-in multiple endocrine neoplasia type B mice induces profound elevation of brain dopamine concentration via enhanced synthesis and increases the number of TH-positive cells in the substantia nigra. J Neurosci 2007, 27:4799-4809.

24. Karperien A, Ahammer H, Jelinek HF: Quantitating the subtleties of microglial morphology with fractal analysis. Front Cell Neurosci 2013, 7:3.

25. Morrison H, Young K, Qureshi M, Rowe RK, Lifshitz J: Quantitative microglia analyses reveal diverse morphologic responses in the rat cortex after diffuse brain injury. Sci Rep 2017, 7:13211.

26. Ferreira TA, Blackman AV, Oyrer J, Jayabal S, Chung AJ, Watt AJ, Sjostrom PJ, van Meyel DJ: Neuronal morphometry directly from bitmap images. Nat Methods 2014, 11:982-984. 
27. Heindl S, Gesierich B, Benakis C, Llovera G, Duering M, Liesz A: Automated Morphological Analysis of Microglia After Stroke. Front Cell Neurosci 2018, 12:106.

28. Huang H-Y, Chang H-F, Tsai M-J, Chen J-S, Wang M-J: 6-Mercaptopurine attenuates tumor necrosis factor-a production in microglia through Nur77-mediated transrepression and PI3K/Akt/mTOR signaling-mediated translational regulation. Journal of neuroinflammation 2016, 13:1-20.

29. Zhou Z, Zhang J, Li X, Xia C, Han Y, Chen H: Protein microarray analysis identifies key cytokines associated with malignant middle cerebral artery infarction. Brain Behav 2017, 7:e00746.

30. Tusher VG, Tibshirani R, Chu G: Significance analysis of microarrays applied to the ionizing radiation response. Proc Natl Acad Sci U S A 2001, 98:5116-5121.

31. Yu G, Wang LG, Han Y, He QY: clusterProfiler: an R package for comparing biological themes among gene clusters. OMICS 2012, 16:284-287.

32. Ewels $P$, Magnusson M, Lundin S, Kaller M: MultiQC: summarize analysis results for multiple tools and samples in a single report. Bioinformatics 2016, 32:3047-3048.

33. Bolger AM, Lohse M, Usadel B: Trimmomatic: a flexible trimmer for Illumina sequence data. Bioinformatics 2014, 30:2114-2120.

34. Kim D, Langmead B, Salzberg SL: HISAT: a fast spliced aligner with low memory requirements. Nat Methods 2015, 12:357-360.

35. Sahraeian SME, Mohiyuddin M, Sebra R, Tilgner H, Afshar PT, Au KF, Bani Asadi N, Gerstein MB, Wong WH, Snyder MP, et al: Gaining comprehensive biological insight into the transcriptome by performing a broad-spectrum RNA-seq analysis. Nat Commun 2017, 8:59.

36. Liao Y, Smyth GK, Shi W: featureCounts: an efficient general purpose program for assigning sequence reads to genomic features. Bioinformatics 2014, 30:923-930.

37. Wang $L$, Feng $Z$, Wang $X$, Wang $X$, Zhang X: DEGseq: an $R$ package for identifying differentially expressed genes from RNA-seq data. Bioinformatics 2010, 26:136-138.

38. Love MI, Huber W, Anders S: Moderated estimation of fold change and dispersion for RNA-seq data with DESeq2. Genome Bio/ 2014, 15:550.

39. Schurch NJ, Schofield P, Gierlinski M, Cole C, Sherstnev A, Singh V, Wrobel N, Gharbi K, Simpson GG, Owen-Hughes T, et al: How many biological replicates are needed in an RNA-seq experiment and which differential expression tool should you use? RNA 2016, 22:839-851.

40. Anders S, McCarthy DJ, Chen Y, Okoniewski M, Smyth GK, Huber W, Robinson MD: Count-based differential expression analysis of RNA sequencing data using R and Bioconductor. Nat Protoc 2013, 8:1765-1786.

41. Li B, Dong C, Li P, Ren Z, Wang H, Yu F, Ning C, Liu K, Wei W, Huang R, et al: Identification of candidate genes associated with porcine meat color traits by genome-wide transcriptome analysis. Sci Rep 2016, 6:35224.

42. Maza E: In Papyro Comparison of TMM (edgeR), RLE (DESeq2), and MRN Normalization Methods for a Simple Two-Conditions-Without-Replicates RNA-Seq Experimental Design. Front Genet 2016, 
7:164.

43. Kanehisa M, Araki M, Goto S, Hattori M, Hirakawa M, Itoh M, Katayama T, Kawashima S, Okuda S, Tokimatsu T, Yamanishi Y: KEGG for linking genomes to life and the environment. Nucleic Acids Res 2008, 36:D480-484.

44. Kanehisa M, Sato Y, Furumichi M, Morishima K, Tanabe M: New approach for understanding genome variations in KEGG. Nucleic Acids Res 2019, 47:D590-D595.

45. Subramanian A, Tamayo P, Mootha VK, Mukherjee S, Ebert BL, Gillette MA, Paulovich A, Pomeroy SL, Golub TR, Lander ES, Mesirov JP: Gene set enrichment analysis: a knowledge-based approach for interpreting genome-wide expression profiles. Proc Natl Acad Sci U S A 2005, 102:15545-15550.

46. Liberzon A, Birger C, Thorvaldsdottir H, Ghandi M, Mesirov JP, Tamayo P: The Molecular Signatures Database (MSigDB) hallmark gene set collection. Cell Syst 2015, 1:417-425.

47. Liberzon A, Subramanian A, Pinchback R, Thorvaldsdottir H, Tamayo P, Mesirov JP: Molecular signatures database (MSigDB) 3.0. Bioinformatics 2011, 27:1739-1740.

48. Langfelder P, Horvath S: WGCNA: an R package for weighted correlation network analysis. $B M C$ Bioinformatics 2008, 9:559.

49. Zhang B, Horvath S: A general framework for weighted gene co-expression network analysis. Stat Appl Genet Mol Biol 2005, 4:Article17.

50. Suda S, Katsura K, Kanamaru T, Saito M, Katayama Y: Valproic acid attenuates ischemia-reperfusion injury in the rat brain through inhibition of oxidative stress and inflammation. Eur J Pharmacol 2013, 707:26-31.

51. Stanzione R, Cotugno M, Bianchi F, Marchitti S, Forte M, Volpe M, Rubattu S: Pathogenesis of Ischemic Stroke: Role of Epigenetic Mechanisms. Genes (Basel) 2020, 11.

52. Li ML, Su XM, Ren Y, Zhao X, Kong LF, Kang J: HDAC8 inhibitor attenuates airway responses to antigen stimulus through synchronously suppressing galectin-3 expression and reducing macrophage-2 polarization. Respir Res 2020, 21:62.

53. Li S, Lu X, Shao Q, Chen Z, Huang Q, Jiao Z, Huang X, Yue M, Peng J, Zhou X, et al: Early Histone Deacetylase Inhibition Mitigates Ischemia/Reperfusion Brain Injury by Reducing Microglia Activation and Modulating Their Phenotype. Front Neurol 2019, 10:893.

54. van Vliet SJ, Garcia-Vallejo JJ, van Kooyk Y: Dendritic cells and C-type lectin receptors: coupling innate to adaptive immune responses. Immunol Cell Biol 2008, 86:580-587.

55. Ilarregui JM, Kooij G, Rodriguez E, van der Pol SMA, Koning N, Kalay H, van der Horst JC, van Vliet SJ, Garcia-Vallejo JJ, de Vries HE, van Kooyk Y: Macrophage galactose-type lectin (MGL) is induced on M2 microglia and participates in the resolution phase of autoimmune neuroinflammation. $J$ Neuroinflammation 2019, 16:130.

56. Hu Z, Zhong B, Tan J, Chen C, Lei Q, Zeng L: The Emerging Role of Epigenetics in Cerebral Ischemia. Mol Neurobiol 2017, 54:1887-1905. 
57. Hamer HM, Knake S, Schomburg U, Rosenow F: Valproate-induced hyperammonemic encephalopathy in the presence of topiramate. Neurology 2000, 54:230-232.

58. Attilakos A, Katsarou E, Voudris K, Garoufi A: Valproate-associated coagulopathies are frequent and variable in children. Epilepsia 2007, 48:205-206.

59. Gotlib D, Ramaswamy R, Kurlander JE, DeRiggi A, Riba M: Valproic Acid in Women and Girls of Childbearing Age. Curr Psychiatry Rep 2017, 19:58.

60. Lee JT, Chou CH, Cho NY, Sung YF, Yang FC, Chen CY, Lai YH, Chiang Cl, Chu CM, Lin JC, et al: Postinsult valproate treatment potentially improved functional recovery in patients with acute middle cerebral artery infarction. Am J Transl Res 2014, 6:820-830.

61. Glass CK, Saijo K, Winner B, Marchetto MC, Gage FH: Mechanisms underlying inflammation in neurodegeneration. Cell 2010, 140:918-934.

62. Suzuki S, Tanaka K, Nogawa S, Nagata E, Ito D, Dembo T, Fukuuchi Y: Temporal profile and cellular localization of interleukin-6 protein after focal cerebral ischemia in rats. J Cereb Blood Flow Metab 1999, 19:1256-1262.

63. Eriksson C, Van Dam AM, Lucassen PJ, Bol JG, Winblad B, Schultzberg M: Immunohistochemical localization of interleukin-1 beta, interleukin-1 receptor antagonist and interleukin-1beta converting enzyme/caspase-1 in the rat brain after peripheral administration of kainic acid. Neuroscience 1999, 93:915-930.

64. Sierra A, de Castro F, Del Rio-Hortega J, Rafael Iglesias-Rozas J, Garrosa M, Kettenmann H: The "BigBang" for modern glial biology: Translation and comments on Pio del Rio-Hortega 1919 series of papers on microglia. Glia 2016, 64:1801-1840.

65. Norris GT, Kipnis J: Immune cells and CNS physiology: Microglia and beyond. J Exp Med 2019, 216:60-70.

66. Lalancette-Hebert M, Swarup V, Beaulieu JM, Bohacek I, Abdelhamid E, Weng YC, Sato S, Kriz J: Galectin-3 is required for resident microglia activation and proliferation in response to ischemic injury. J Neurosci 2012, 32:10383-10395.

67. Reichert F, Rotshenker S: Galectin-3 (MAC-2) Controls Microglia Phenotype Whether Amoeboid and Phagocytic or Branched and Non-phagocytic by Regulating the Cytoskeleton. Front Cell Neurosci 2019, 13:90.

68. Hoyos HC, Rinaldi M, Mendez-Huergo SP, Marder M, Rabinovich GA, Pasquini JM, Pasquini LA: Galectin-3 controls the response of microglial cells to limit cuprizone-induced demyelination. Neurobiol Dis 2014, 62:441-455.

69. Nomura K, Vilalta A, Allendorf DH, Hornik TC, Brown GC: Activated Microglia Desialylate and Phagocytose Cells via Neuraminidase, Galectin-3, and Mer Tyrosine Kinase. J Immunol 2017, 198:4792-4801.

70. Siew JJ, Chen HM, Chen HY, Chen HL, Chen CM, Soong BW, Wu YR, Chang CP, Chan YC, Lin CH, et al: Galectin-3 is required for the microglia-mediated brain inflammation in a model of Huntington's disease. Nat Commun 2019, 10:3473. 
71. Hsu DK, Hammes SR, Kuwabara I, Greene WC, Liu FT: Human T lymphotropic virus-I infection of human T lymphocytes induces expression of the beta-galactoside-binding lectin, galectin-3. Am J Pathol 1996, 148:1661-1670.

72. Li Z, Lv F, Dai C, Wang Q, Jiang C, Fang M, Xu Y: Activation of Galectin-3 (LGALS3) Transcription by Injurious Stimuli in the Liver Is Commonly Mediated by BRG1. Front Cell Dev Bio/ 2019, 7:310.

73. Nikolian VC, Dennahy IS, Higgins GA, Williams AM, Weykamp M, Georgoff PE, Eidy H, Ghandour MH, Chang P, Alam HB: Transcriptomic changes following valproic acid treatment promote neurogenesis and minimize secondary brain injury. J Trauma Acute Care Surg 2018, 84:459-465.

74. Ucar B, Humpel C: Collagen for brain repair: therapeutic perspectives. Neural Regen Res 2018, 13:595-598.

75. Gregorio I, Braghetta P, Bonaldo P, Cescon M: Collagen VI in healthy and diseased nervous system. Dis Model Mech 2018, 11.

76. Davis GE, Senger DR: Endothelial extracellular matrix: biosynthesis, remodeling, and functions during vascular morphogenesis and neovessel stabilization. Circ Res 2005, 97:1093-1107.

77. Kubota S, Takigawa M: CCN family proteins and angiogenesis: from embryo to adulthood. Angiogenesis 2007, 10:1-11.

78. Jarvelainen H, Sainio A, Wight TN: Pivotal role for decorin in angiogenesis. Matrix Bio/ 2015, 43:1526.

79. Nelimarkka L, Salminen H, Kuopio T, Nikkari S, Ekfors T, Laine J, Pelliniemi L, Jarvelainen H: Decorin is produced by capillary endothelial cells in inflammation-associated angiogenesis. Am J Pathol 2001, 158:345-353.

80. Wenzel J, Assmann JC, Schwaninger M: Thrombomodulin-a new target for treating stroke at the crossroad of coagulation and inflammation. Curr Med Chem 2014, 21:2025-2034.

81. Watanabe-Kusunoki K, Nakazawa D, Ishizu A, Atsumi T: Thrombomodulin as a Physiological Modulator of Intravascular Injury. Front Immunol 2020, 11:575890.

82. Cherry JD, Olschowka JA, O'Banion MK: Neuroinflammation and M2 microglia: the good, the bad, and the inflamed. $J$ Neuroinflammation 2014, 11:98.

83. Etzerodt A, Moestrup SK: CD163 and inflammation: biological, diagnostic, and therapeutic aspects. Antioxid Redox Signal 2013, 18:2352-2363.

84. Taylor RA, Chang CF, Goods BA, Hammond MD, Mac Grory B, Ai Y, Steinschneider AF, Renfroe SC, Askenase MH, McCullough LD, et al: TGF-beta1 modulates microglial phenotype and promotes recovery after intracerebral hemorrhage. J Clin Invest 2017, 127:280-292.

\section{Figures}


A
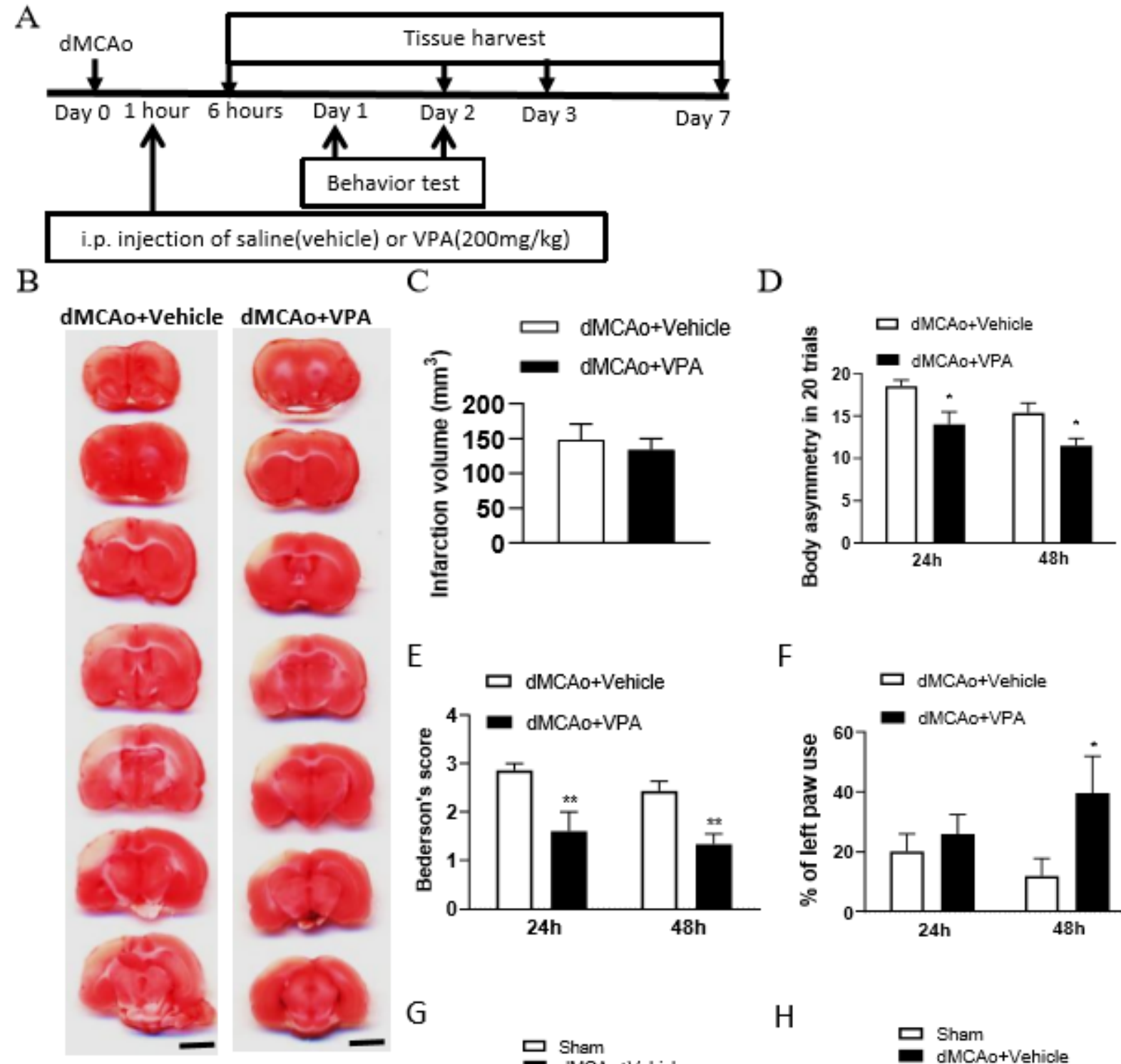

$\mathrm{E}$
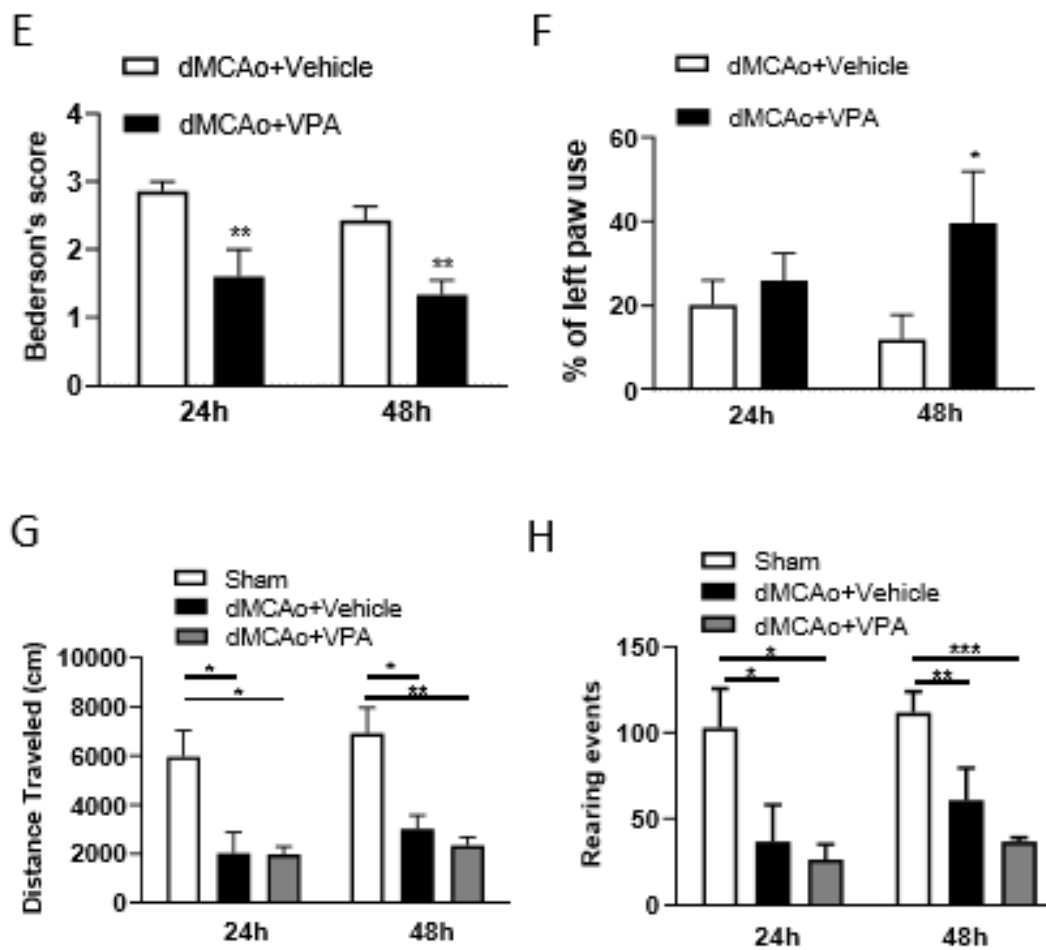

$\mathrm{H}$

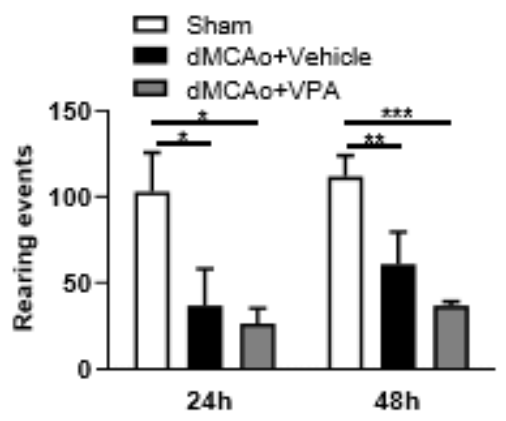

Figure 1

Post-stroke single injection of VPA $(200 \mathrm{mg} / \mathrm{kg})$ does not reduce infarct area, but promotes functional recovery. A, experimental timeline. The rats underwent dMCAo surgery, and they were divided into two groups randomly. One hour after reperfusion, the animals received an intraperitoneal injection of either VPA or vehicle once, and their behavioral functions were evaluated on day 1 and 2 . Rats were sacrificed for analysis at different time points. B, a representative schematic diagram showing infarct area stained with TTC in a series of coronal sections derived from these two groups of rats killed at 48 hours after 
ischemia. $C$, quantified results of infarct volumes derived from animals killed at 48 hours after the onset of dMCAO. D, E, effects of VPA $(n=7)$, vehicle $(n=8)$ on body asymmetry (D), Bederson's neurologic test score $(E),{ }^{*} p<0.05,{ }^{*} p<0.01$ indicate comparison with vehicle with Bonferroni's post hoc test following two-way ANOVA. F, forepaw use bias of the rats was assessed using the cylinder test on day 1 and 2 after dMCAo. ${ }^{*} p=0.039$ by Bonferroni's multiple comparisons test, following two-way ANOVA. G, H, effects of VPA $(n=5)$, vehicle $(n=6)$ and no treatment $(n=4)$ on vertical $(G)$ and horizontal $(H)$ activity measured for 30 minutes on day 1 and $2 .{ }^{*} p<0.05,{ }^{* *} p<0.01,{ }^{* \star *} p<0.001$ by Bonferroni's multiple comparisons test, following one-way ANOVA. Scale bar: $5000 \mu \mathrm{m}$. The data represent mean \pm SEM.

A

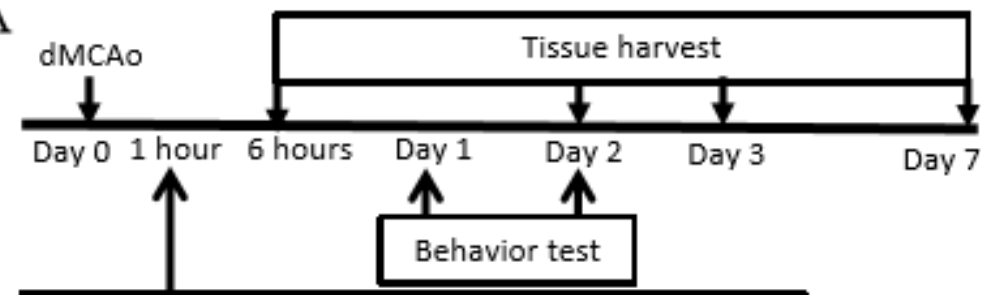

i.p. injection of saline(vehicle) or VPA $(200 \mathrm{mg} / \mathrm{kg}$ )

B

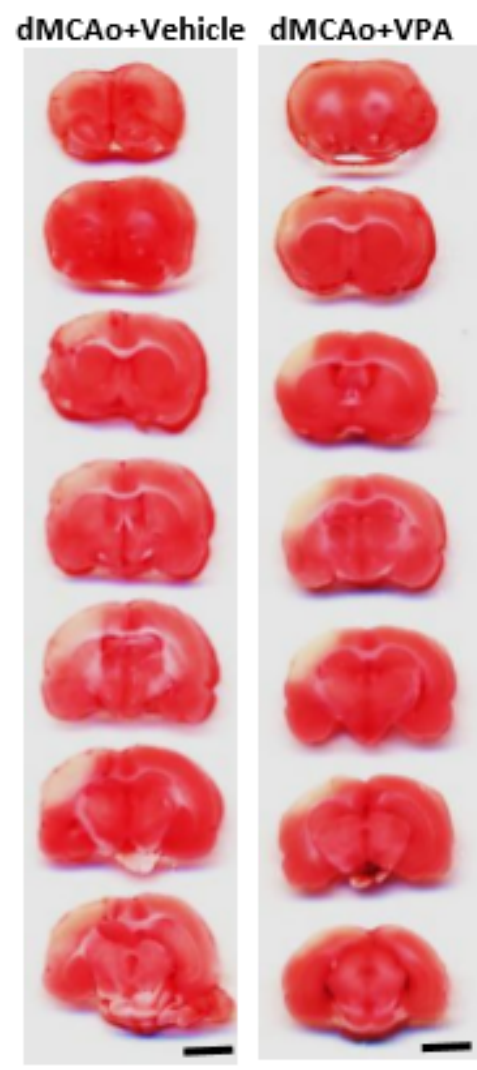

$\mathrm{C}$

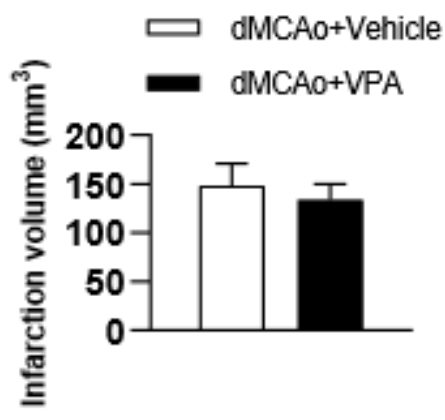

E

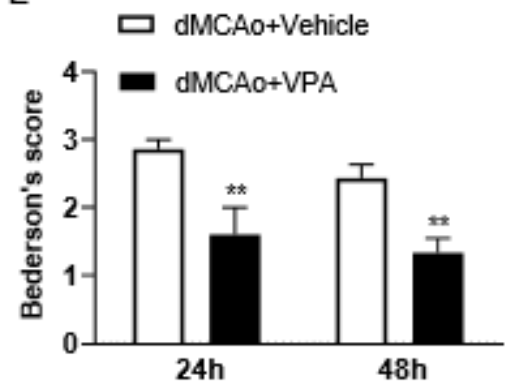

G

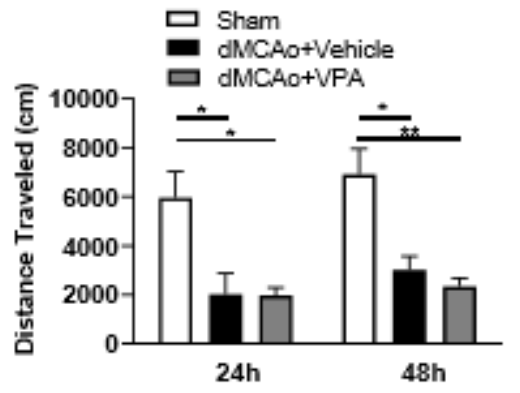

D

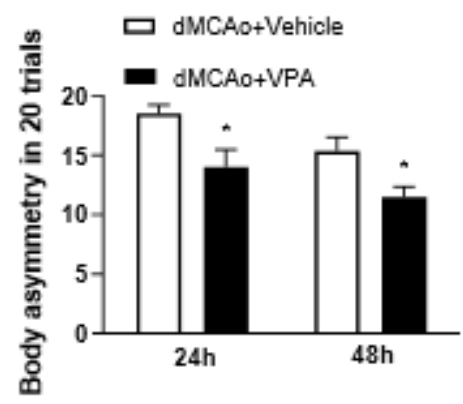

F

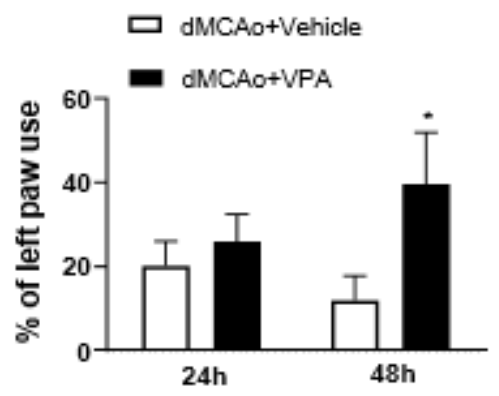

$\mathrm{H}$

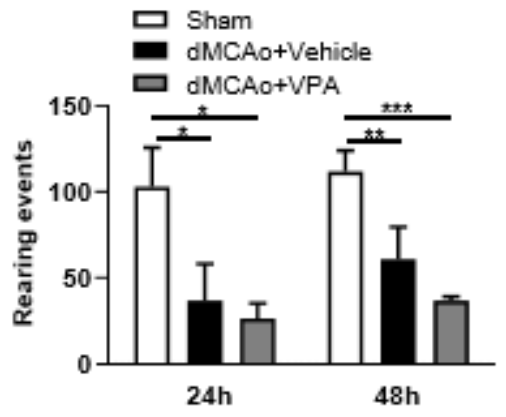




\section{Figure 1}

Post-stroke single injection of VPA $(200 \mathrm{mg} / \mathrm{kg})$ does not reduce infarct area, but promotes functional recovery. A, experimental timeline. The rats underwent dMCAo surgery, and they were divided into two groups randomly. One hour after reperfusion, the animals received an intraperitoneal injection of either VPA or vehicle once, and their behavioral functions were evaluated on day 1 and 2 . Rats were sacrificed for analysis at different time points. B, a representative schematic diagram showing infarct area stained with TTC in a series of coronal sections derived from these two groups of rats killed at 48 hours after ischemia. $C$, quantified results of infarct volumes derived from animals killed at 48 hours after the onset of dMCAo. D, E, effects of VPA $(n=7)$, vehicle $(n=8)$ on body asymmetry (D), Bederson's neurologic test score $(E),{ }^{*}<<0.05,{ }^{*} p<0.01$ indicate comparison with vehicle with Bonferroni's post hoc test following two-way ANOVA. F, forepaw use bias of the rats was assessed using the cylinder test on day 1 and 2 after dMCAo. ${ }^{*} p=0.039$ by Bonferroni's multiple comparisons test, following two-way ANOVA. G, H, effects of VPA $(n=5)$, vehicle $(n=6)$ and no treatment $(n=4)$ on vertical $(G)$ and horizontal $(H)$ activity measured for 30 minutes on day 1 and $2 .{ }^{\star} p<0.05,{ }^{*} p<0.01,{ }^{\star \star *} p<0.001$ by Bonferroni's multiple comparisons test, following one-way ANOVA. Scale bar: $5000 \mu \mathrm{m}$. The data represent mean \pm SEM. 
A
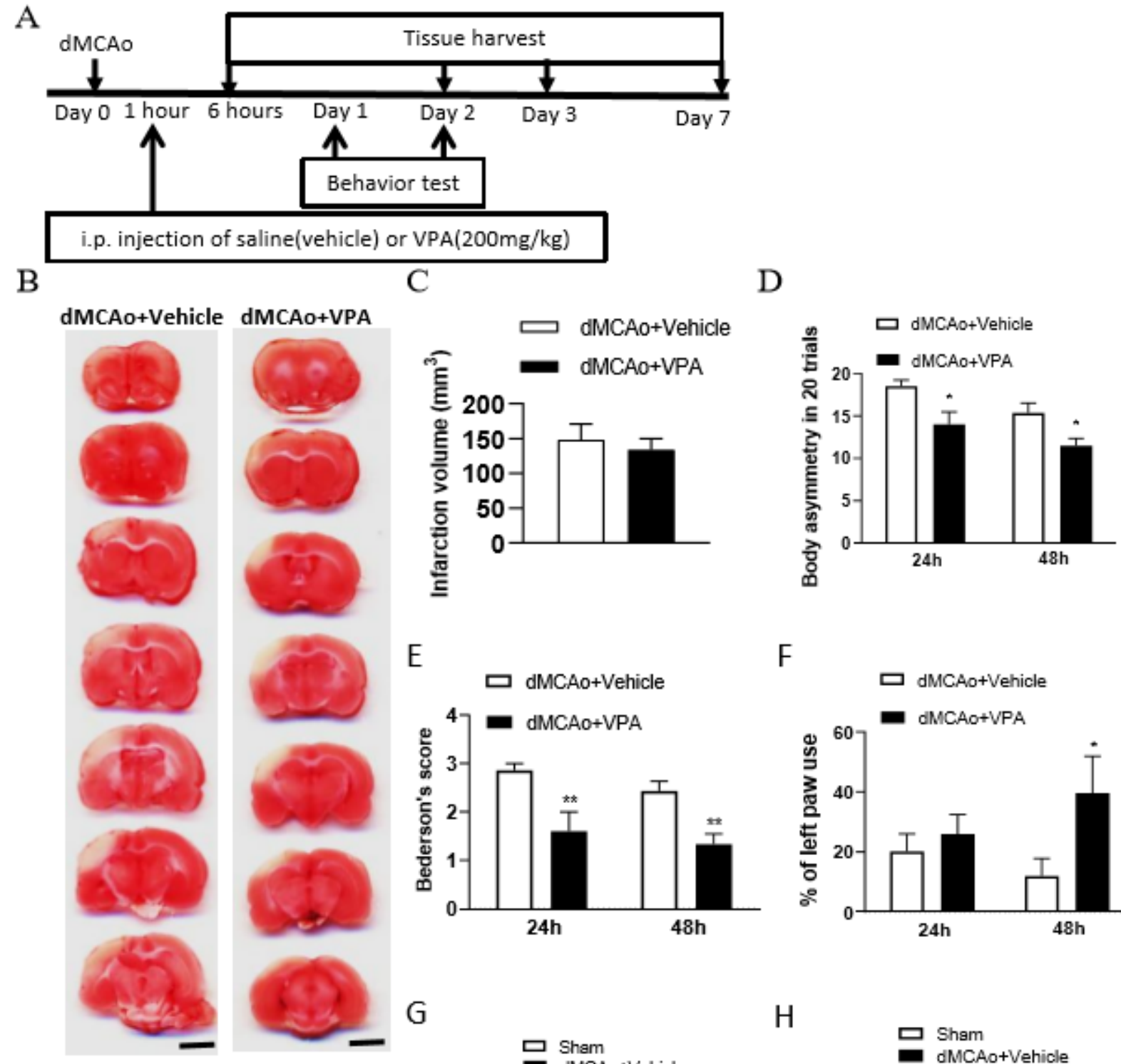

$\mathrm{E}$
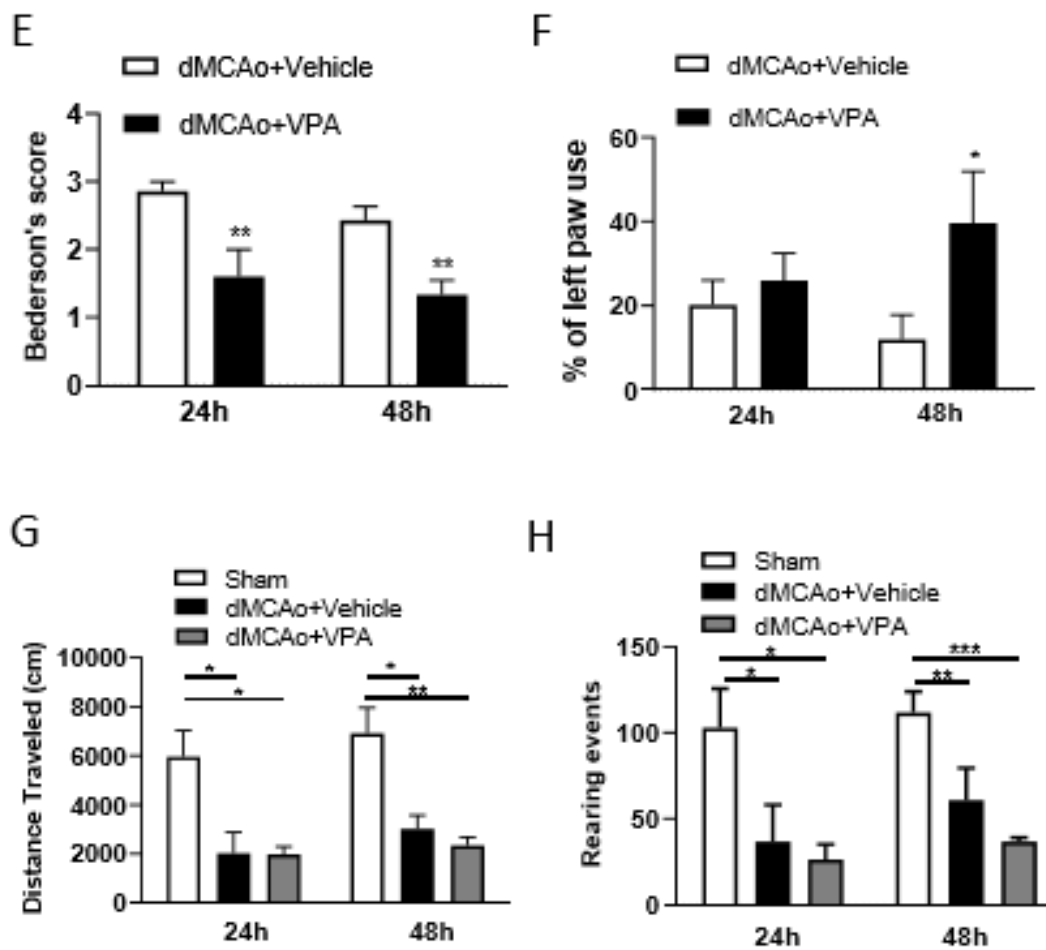

$\mathrm{H}$

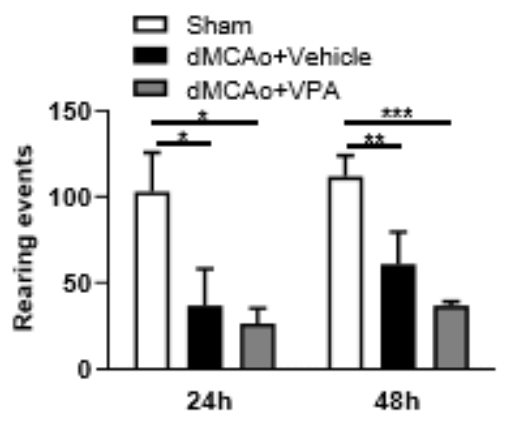

Figure 1

Post-stroke single injection of VPA $(200 \mathrm{mg} / \mathrm{kg})$ does not reduce infarct area, but promotes functional recovery. A, experimental timeline. The rats underwent dMCAo surgery, and they were divided into two groups randomly. One hour after reperfusion, the animals received an intraperitoneal injection of either VPA or vehicle once, and their behavioral functions were evaluated on day 1 and 2 . Rats were sacrificed for analysis at different time points. B, a representative schematic diagram showing infarct area stained with TTC in a series of coronal sections derived from these two groups of rats killed at 48 hours after 
ischemia. $C$, quantified results of infarct volumes derived from animals killed at 48 hours after the onset of dMCAo. D, E, effects of VPA $(n=7)$, vehicle $(n=8)$ on body asymmetry (D), Bederson's neurologic test score $(E),{ }^{*}<<0.05,{ }^{*} p<0.01$ indicate comparison with vehicle with Bonferroni's post hoc test following two-way ANOVA. F, forepaw use bias of the rats was assessed using the cylinder test on day 1 and 2 after dMCAo. ${ }^{*} \mathrm{p}=0.039$ by Bonferroni's multiple comparisons test, following two-way ANOVA. G, H, effects of VPA $(n=5)$, vehicle $(n=6)$ and no treatment $(n=4)$ on vertical $(G)$ and horizontal $(H)$ activity measured for 30 minutes on day 1 and $2 .{ }^{*} p<0.05,{ }^{* \star} p<0.01,{ }^{\star \star \star} p<0.001$ by Bonferroni's multiple comparisons test, following one-way ANOVA. Scale bar: $5000 \mu \mathrm{m}$. The data represent mean \pm SEM. 
A

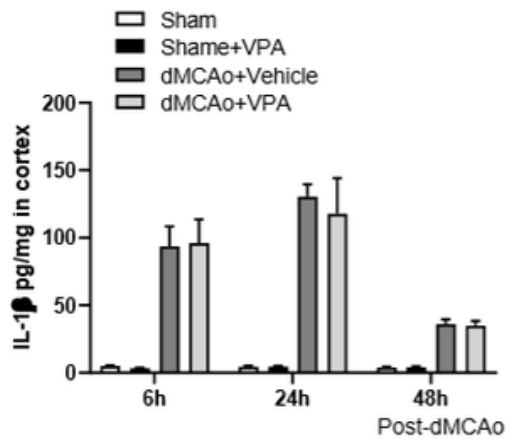

C

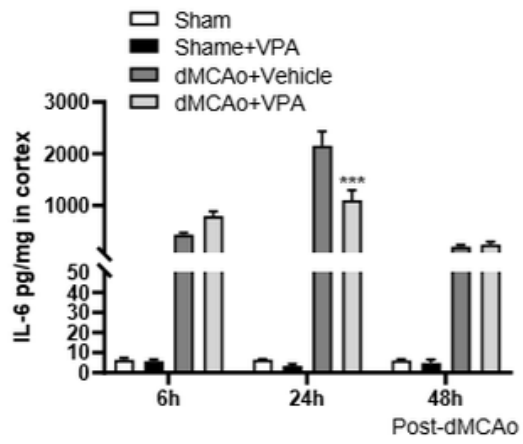

E

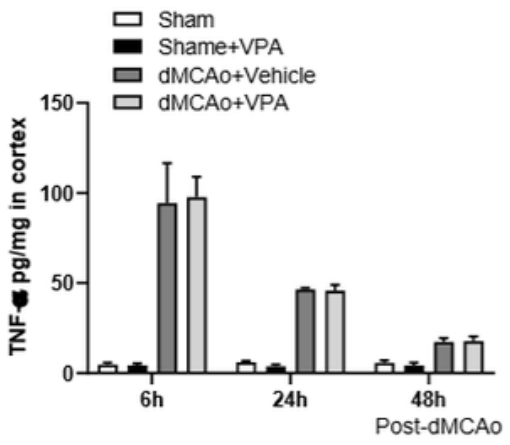

G

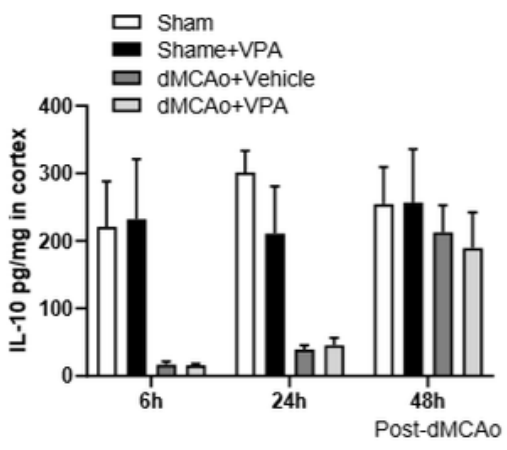

B

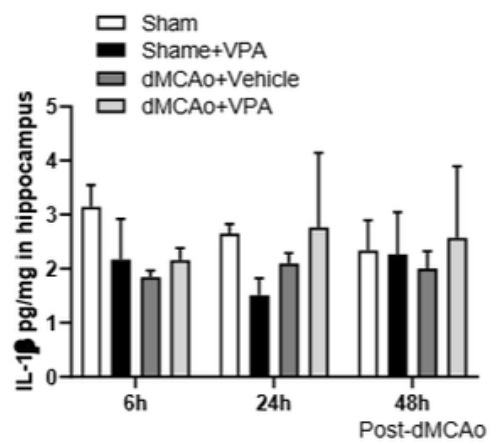

D

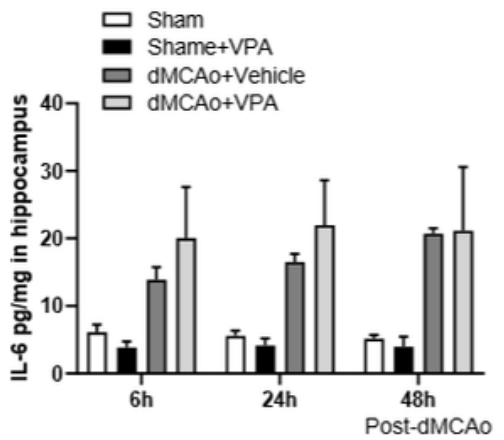

F

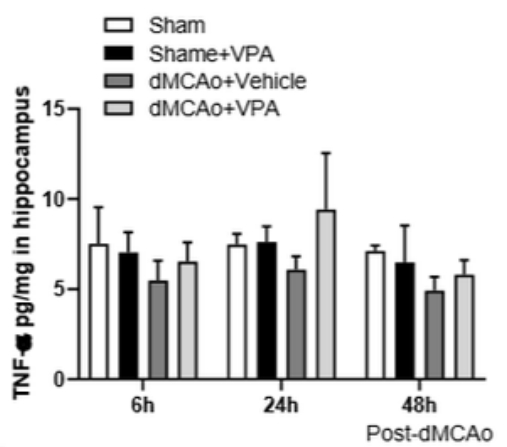

$\mathrm{H}$

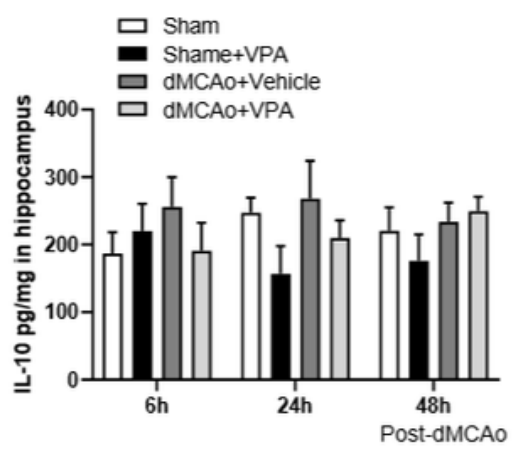

Figure 2

VPA suppresses IL-6 production in the peri-infarct cortex at 24 hours after dMCAo surgery. A, B, IL-1 $\beta$ concentrations in the peri-infarct cortex $(A)$ and ipsilateral hippocampus (B) were measured by ELISA. C, D, TNF-a concentrations in the peri-infarct cortex (C) and ipsilateral hippocampus (D) were measured by ELISA. E, F, IL-6 concentrations in the peri-infarct cortex (E) and ipsilateral hippocampus (F) were measured by ELISA. G, H, IL-10 concentrations in the peri-infarct cortex (G) and ipsilateral hippocampus 
$(\mathrm{H})$ were measured by ELISA. ${ }^{* \star} \mathrm{p}<0.001$ indicates comparison with the dMCAo+ vehicle group with twoway ANOVA, Bonferroni's post hoc test. The data represent mean \pm SEM.

A

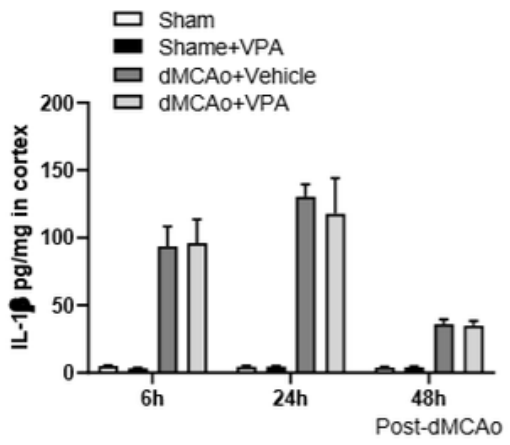

$\mathrm{C}$

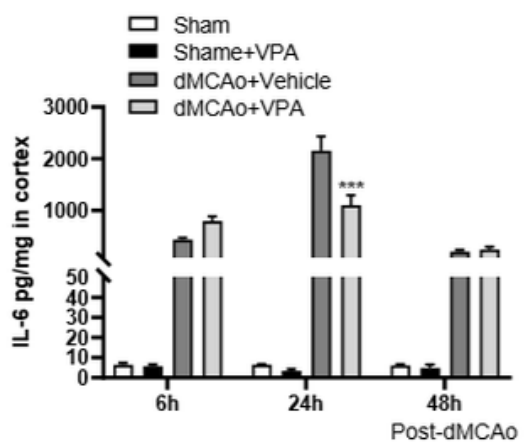

E

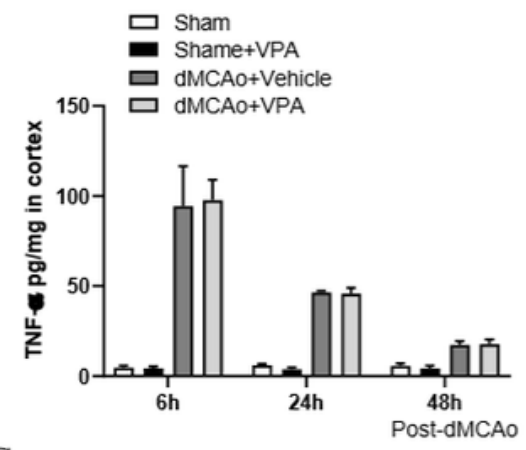

G

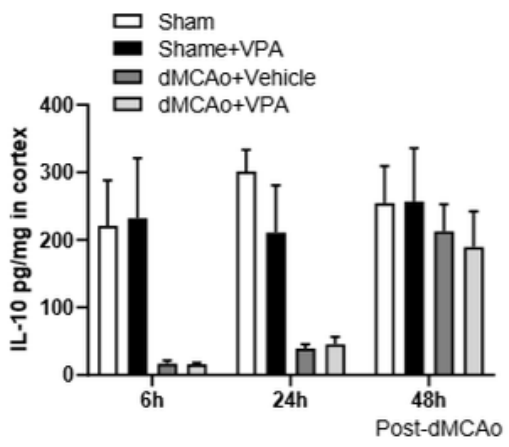

B

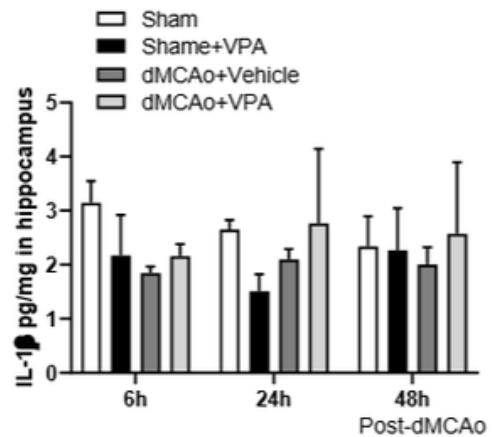

$\mathrm{D}$

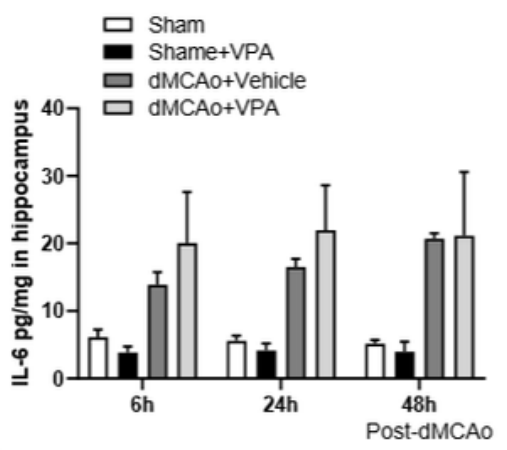

$\mathrm{F}$

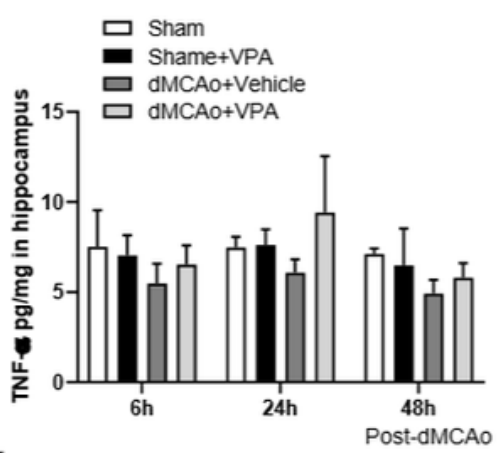

$\mathrm{H}$

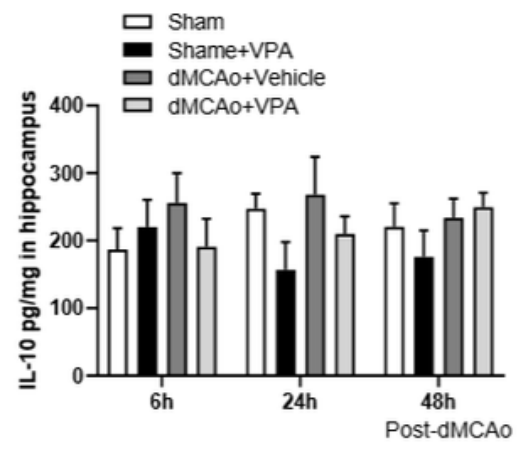

Figure 2

VPA suppresses IL-6 production in the peri-infarct cortex at 24 hours after dMCAo surgery. A, B, IL-1 $\beta$ concentrations in the peri-infarct cortex (A) and ipsilateral hippocampus (B) were measured by ELISA. C, D, TNF-a concentrations in the peri-infarct cortex (C) and ipsilateral hippocampus (D) were measured by 
ELISA. E, F, IL-6 concentrations in the peri-infarct cortex (E) and ipsilateral hippocampus (F) were measured by ELISA. G, H, IL-10 concentrations in the peri-infarct cortex (G) and ipsilateral hippocampus $(\mathrm{H})$ were measured by ELISA. ${ }^{* \star *} \mathrm{p}<0.001$ indicates comparison with the dMCAo+ vehicle group with twoway ANOVA, Bonferroni's post hoc test. The data represent mean \pm SEM.

A

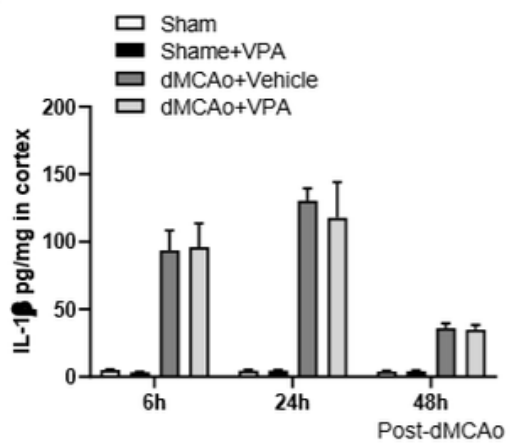

$\mathrm{C}$

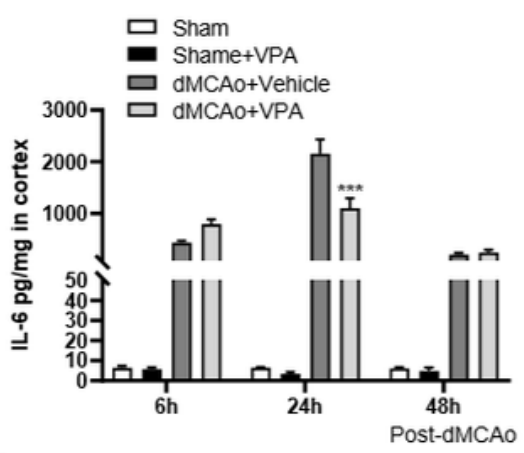

E

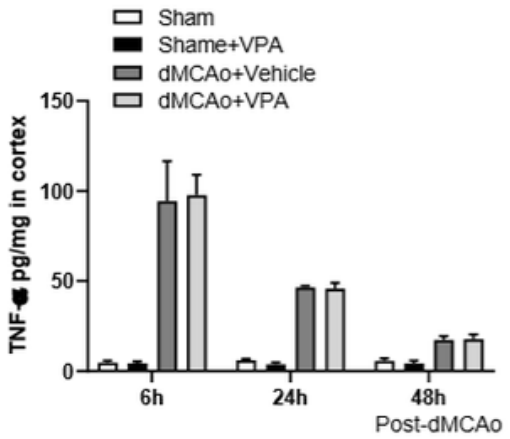

G

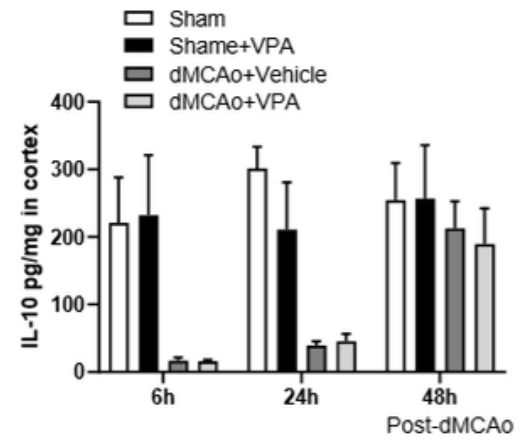

B

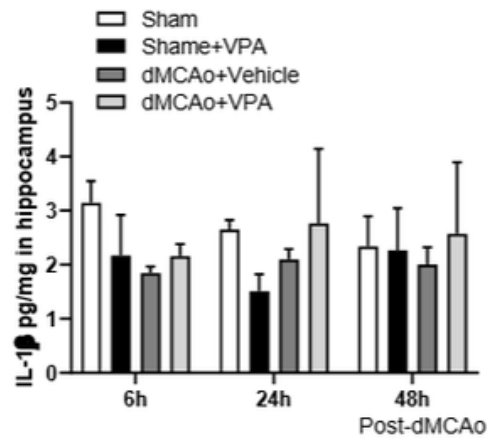

$\mathrm{D}$

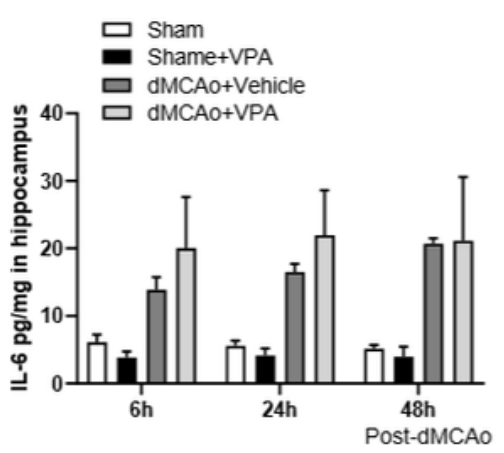

F

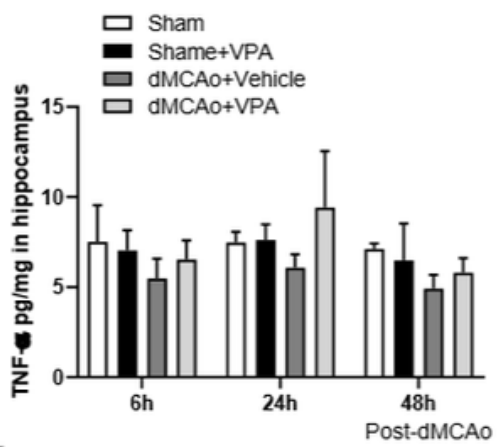

$\mathrm{H}$

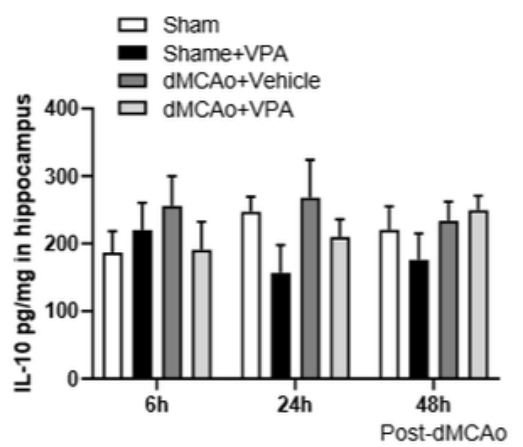

Figure 2 
VPA suppresses IL-6 production in the peri-infarct cortex at 24 hours after dMCAo surgery. A, B, IL-1 $\beta$ concentrations in the peri-infarct cortex (A) and ipsilateral hippocampus (B) were measured by ELISA. C, $D$, TNF-a concentrations in the peri-infarct cortex (C) and ipsilateral hippocampus (D) were measured by ELISA. E, F, IL-6 concentrations in the peri-infarct cortex (E) and ipsilateral hippocampus (F) were measured by ELISA. G, H, IL-10 concentrations in the peri-infarct cortex $(\mathrm{G})$ and ipsilateral hippocampus $(H)$ were measured by ELISA. ${ }^{* \star *} p<0.001$ indicates comparison with the dMCAo+ vehicle group with twoway ANOVA, Bonferroni's post hoc test. The data represent mean \pm SEM.

A

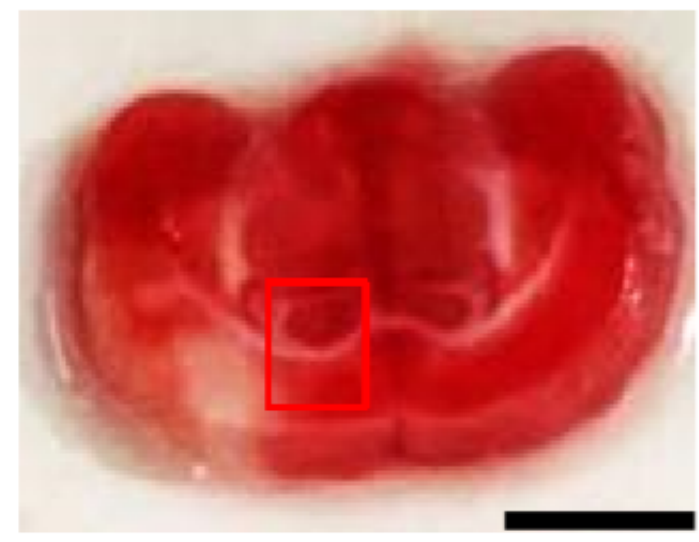

$\mathrm{C}$

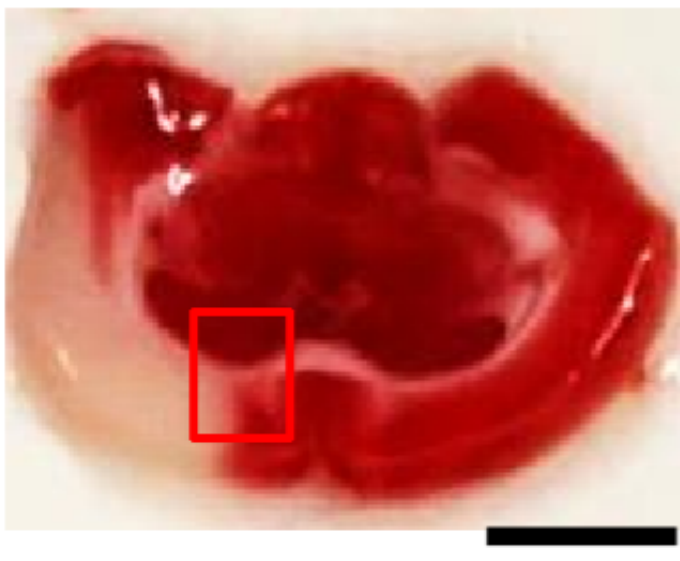

$E$

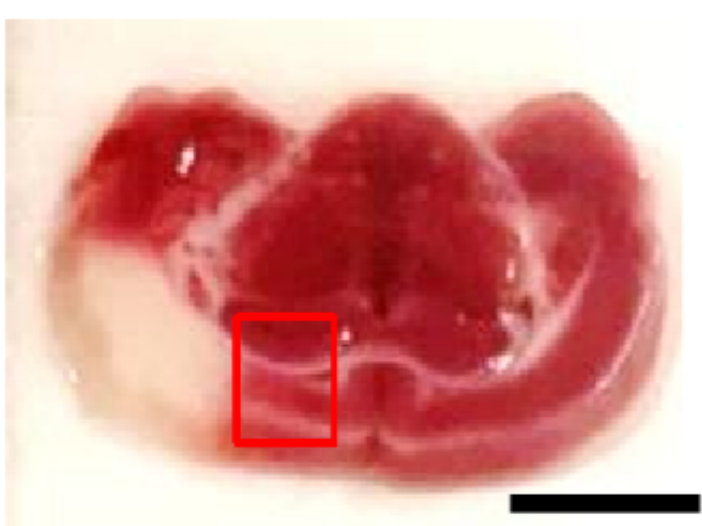

B

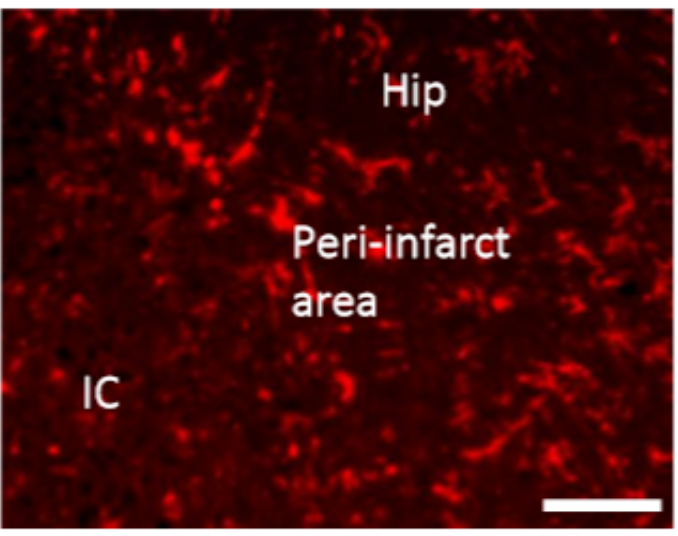

D

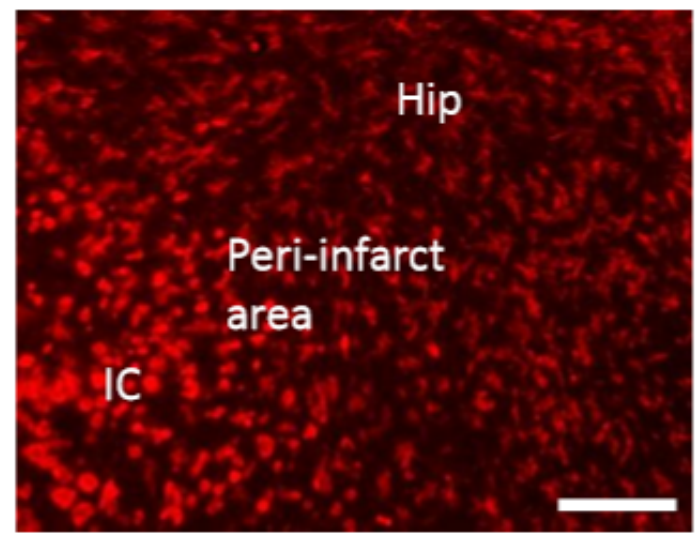

F

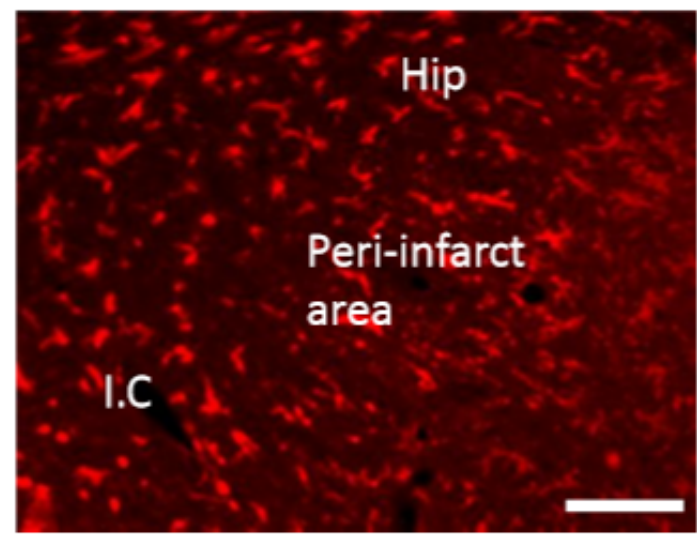




\section{Figure 3}

Time course of microglia/macrophage activation after cortical stroke. Representative images of TTC staining and immunostaining of all microglia/macrophages (CD11b) from ischemic core (IC), peri-infarct area, and hippocampus (Hip) coronal sections at 1 (A, B), 2 (C, D), and 7 (E, F) days after 90-minute $\mathrm{dMCAo}$ in rats. The morphological changes and increasing number of microglia/macrophages were obviously found at 2 days after ischemia. Scale bar is $50 \mu \mathrm{m}$ (immunofluorescence of CD11b) and 5000 $\mu \mathrm{m}$ (TTC staining).

A

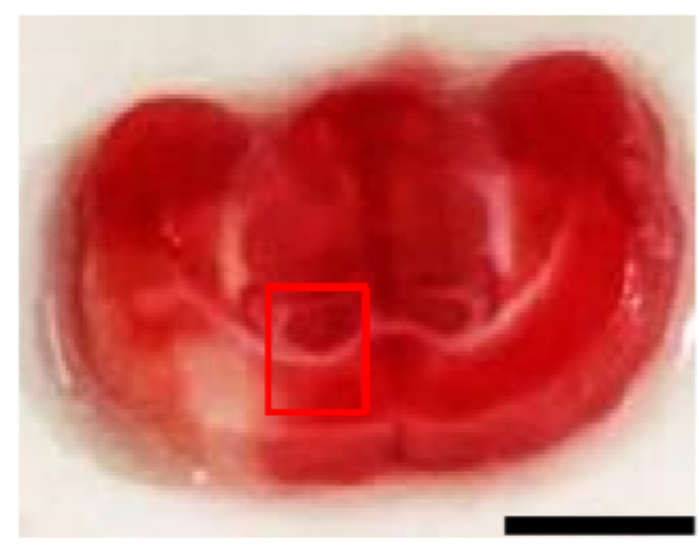

$\mathrm{C}$

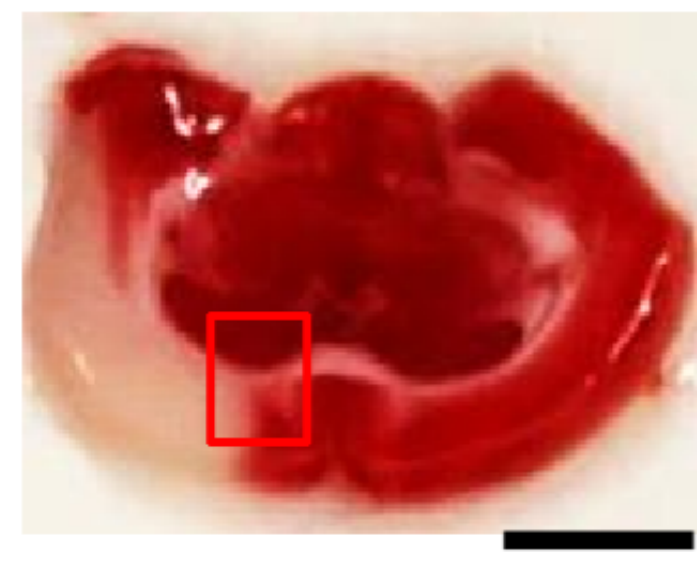

$E$

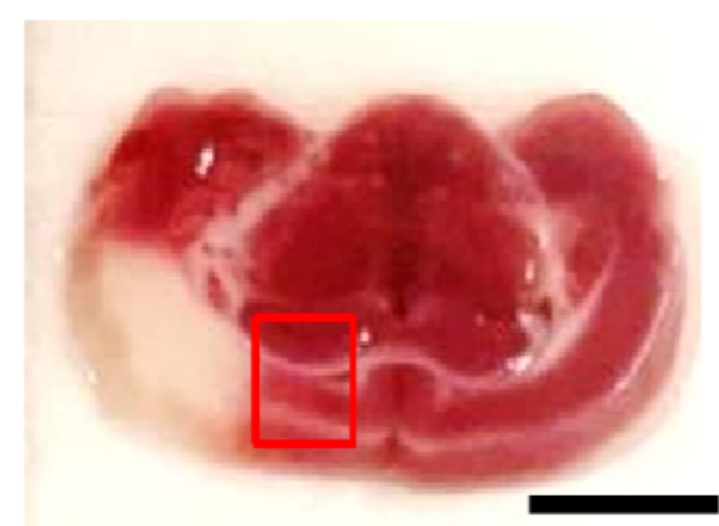

B

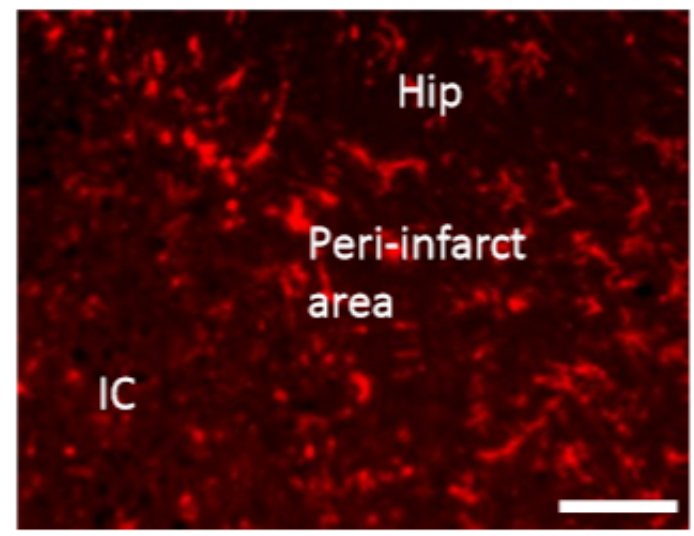

D

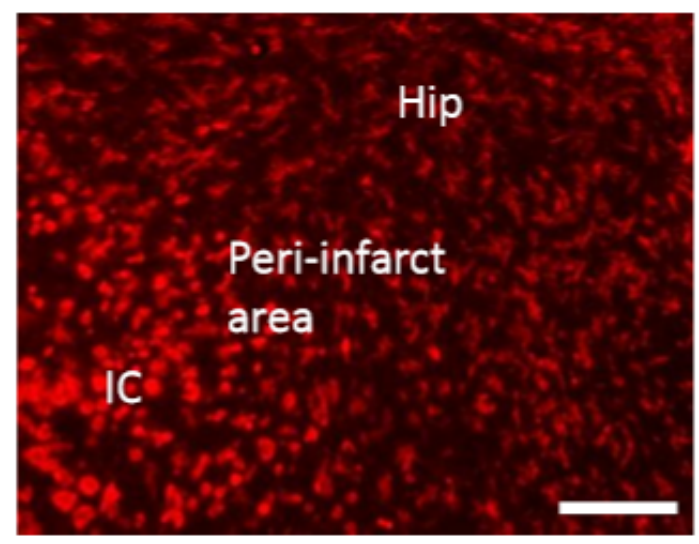

F

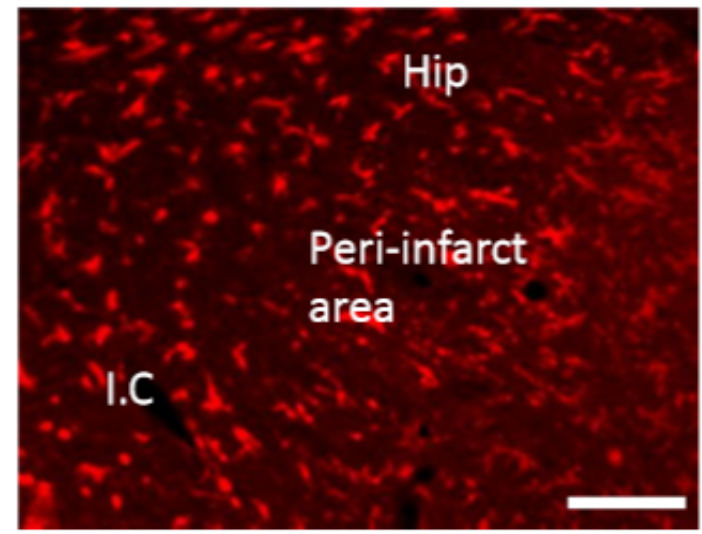




\section{Figure 3}

Time course of microglia/macrophage activation after cortical stroke. Representative images of TTC staining and immunostaining of all microglia/macrophages (CD11b) from ischemic core (IC), peri-infarct area, and hippocampus (Hip) coronal sections at 1 (A, B), 2 (C, D), and 7 (E, F) days after 90-minute $\mathrm{dMCAo}$ in rats. The morphological changes and increasing number of microglia/macrophages were obviously found at 2 days after ischemia. Scale bar is $50 \mu \mathrm{m}$ (immunofluorescence of CD11b) and 5000 $\mu \mathrm{m}$ (TTC staining).

A

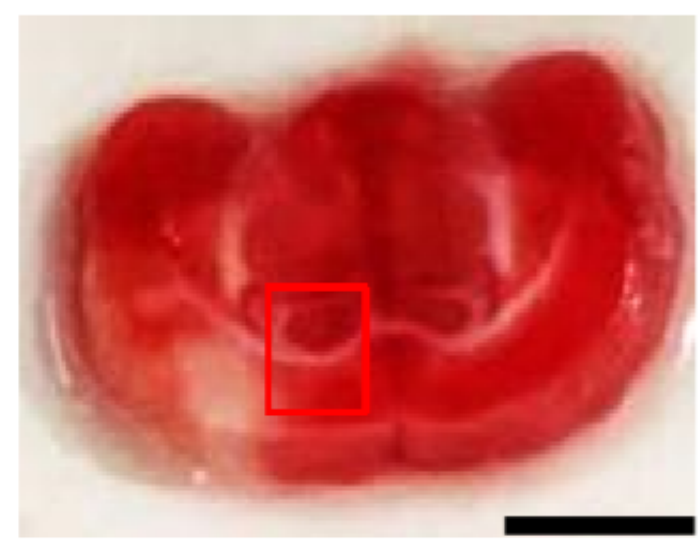

$\mathrm{C}$

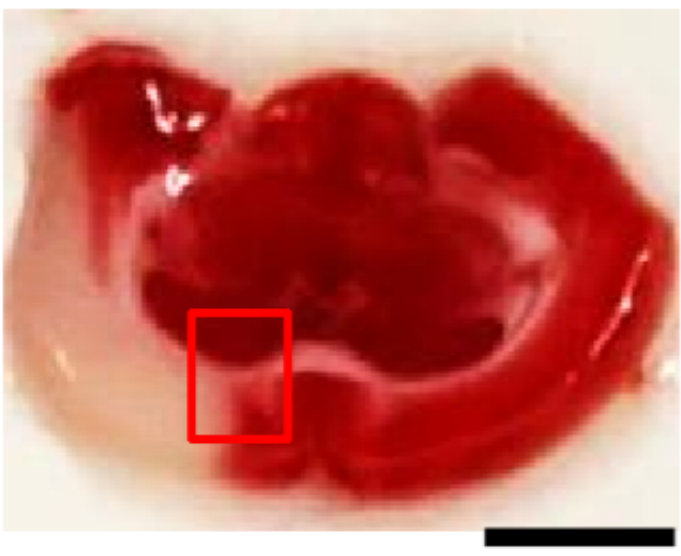

$E$

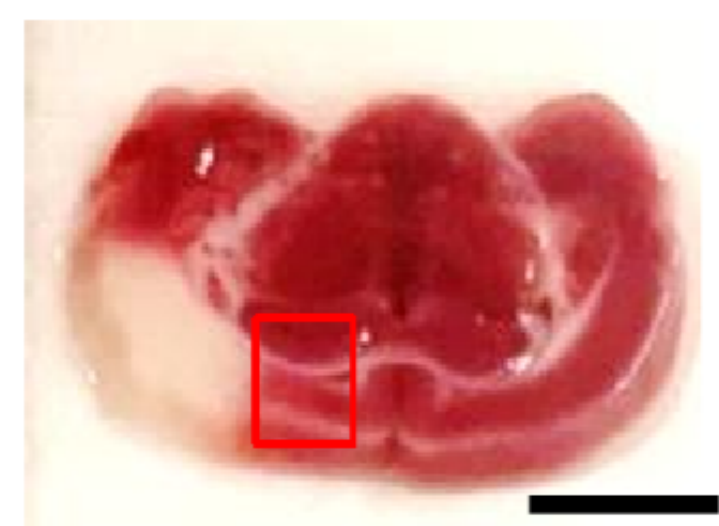

B

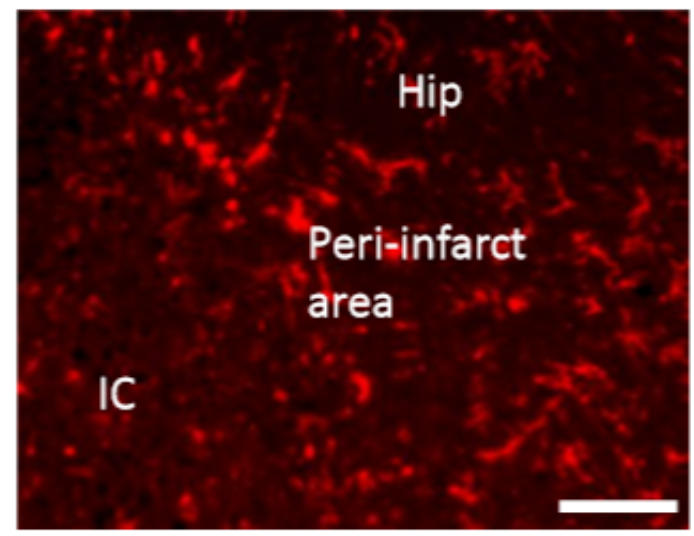

D

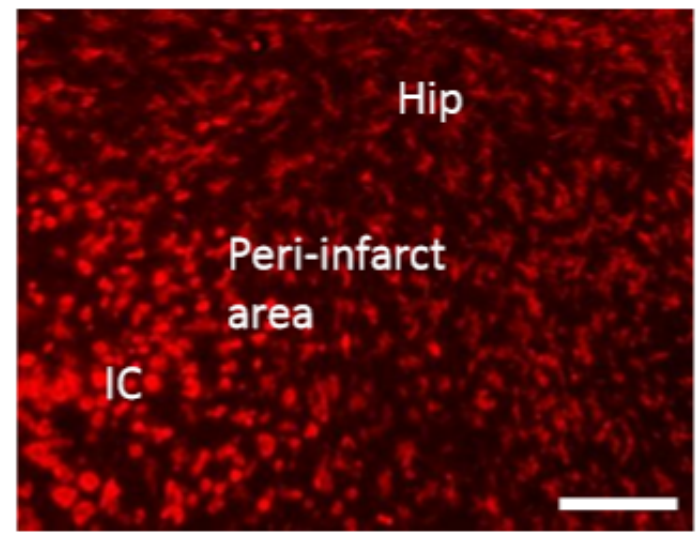

F

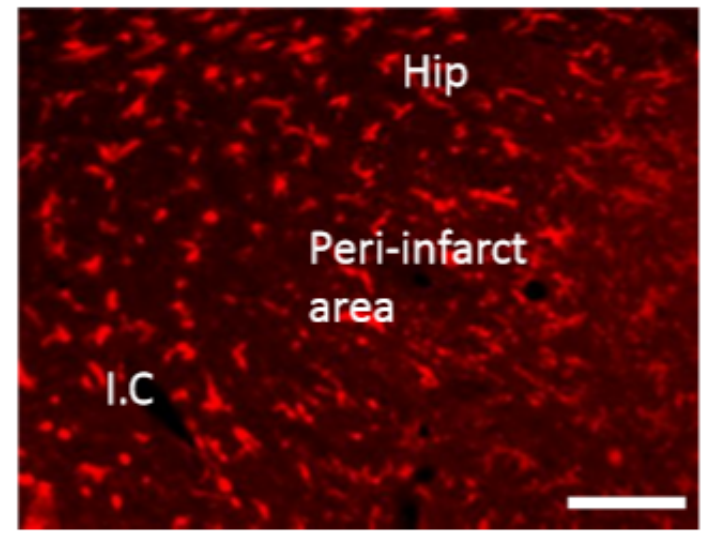




\section{Figure 3}

Time course of microglia/macrophage activation after cortical stroke. Representative images of TTC staining and immunostaining of all microglia/macrophages (CD11b) from ischemic core (IC), peri-infarct area, and hippocampus (Hip) coronal sections at 1 (A, B), 2 (C, D), and 7 (E, F) days after 90-minute $\mathrm{dMCAo}$ in rats. The morphological changes and increasing number of microglia/macrophages were obviously found at 2 days after ischemia. Scale bar is $50 \mu \mathrm{m}$ (immunofluorescence of CD11b) and 5000 $\mu \mathrm{m}$ (TTC staining).
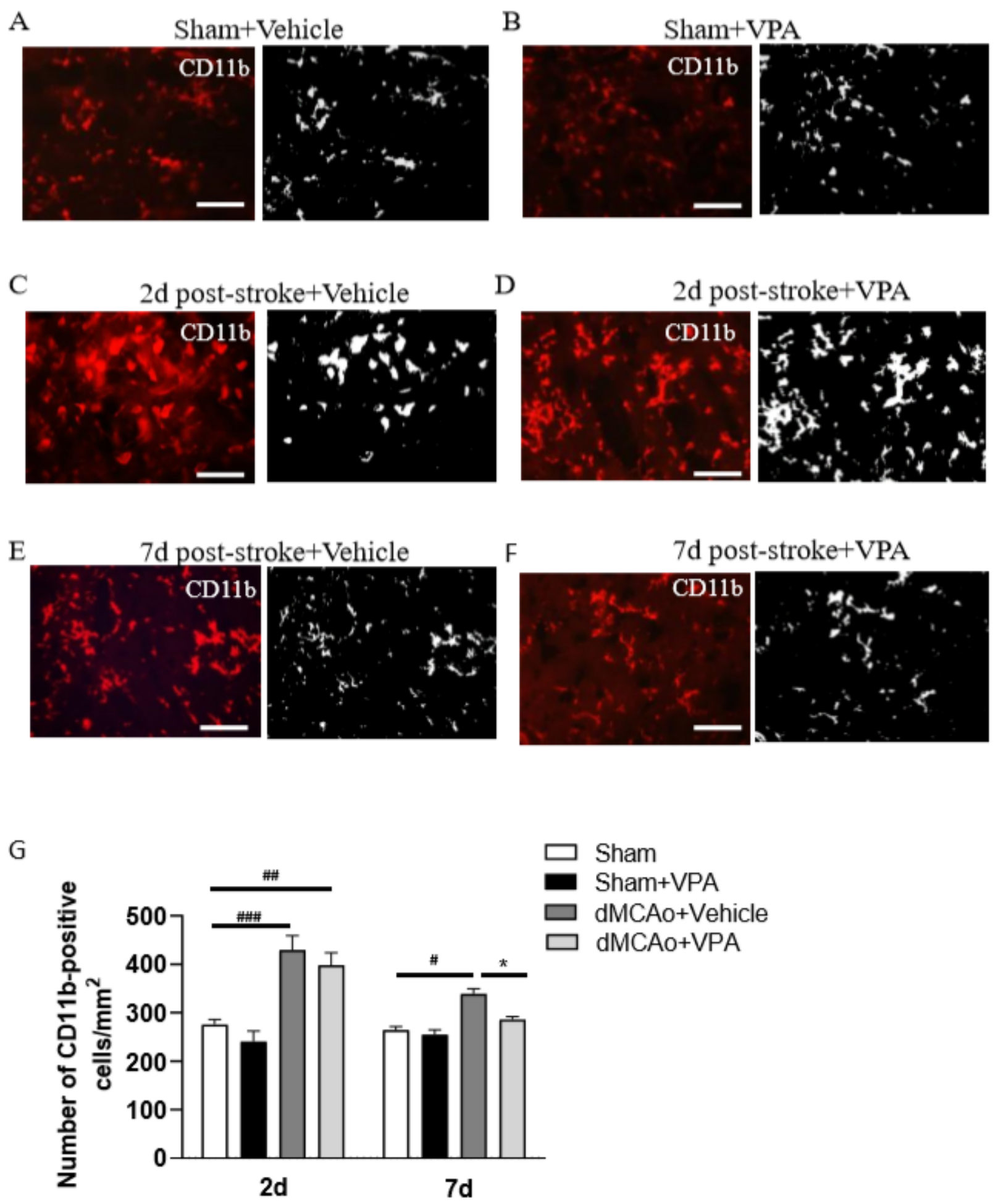


\section{Figure 4}

Post-stroke intraperitoneal injection of VPA decreases the number of CD11b-positive cells in the periinfarct cortex. A-F, representative photomicrographs of anti-CD11b immunofluorescent staining of the sham-operated cortex in vehicle-treated rats (A), sham-operated cortex in VPA-treated rats (B), peri-infarct cortex at 2 days after 90-minute dMCAo in vehicle-treated rats (C), peri-infarct cortex at 2 days after 90minute dMCAo in VPA-treated rats (D), peri-infarct cortex at 7 days after 90-minute dMCAo in vehicletreated rats $(E)$, and peri-infarct cortex at 7 days after 90-minute dMCAo in VPA-treated rats $(F)$. G, quantitation of CD11b-positive cells in the peri-infarct cortex at different time points showing accumulation of activated microglia/macrophages in the peri-infarct cortex at 2 and 7 days after ischemia. Original photomicrographs were subjected to a series of uniform Image $\mathrm{J}$ plugin protocols prior to conversion to binary images; binary images were then analyzed to calculate the number of CD11bpositive cells. $\# p<0.05$, \#\#p< 0.01, and \#\#\# $p<0.001$ indicate statistical difference between peri-infarct cortex and sham-operated cortex at each time point, Bonferroni's multiple comparisons test, following one-way ANOVA. * $p<0.05$ indicates comparison with the dMCAo+ vehicle group with two-way ANOVA, Bonferroni's post hoc test. Five tissue sections per rat were used for the analysis $(n=8-10)$. Scale bar: 20 $\mu \mathrm{m}$. The data represent mean \pm SEM. 
A
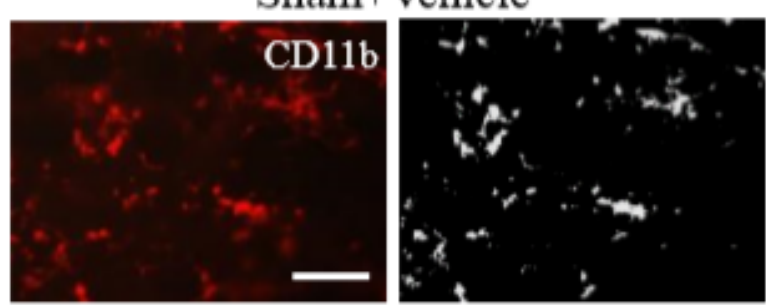

B

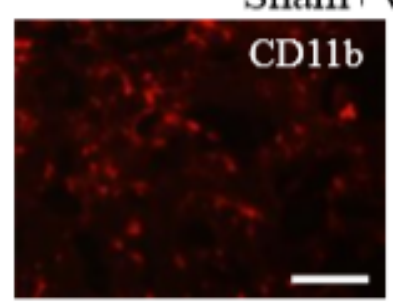

D

C
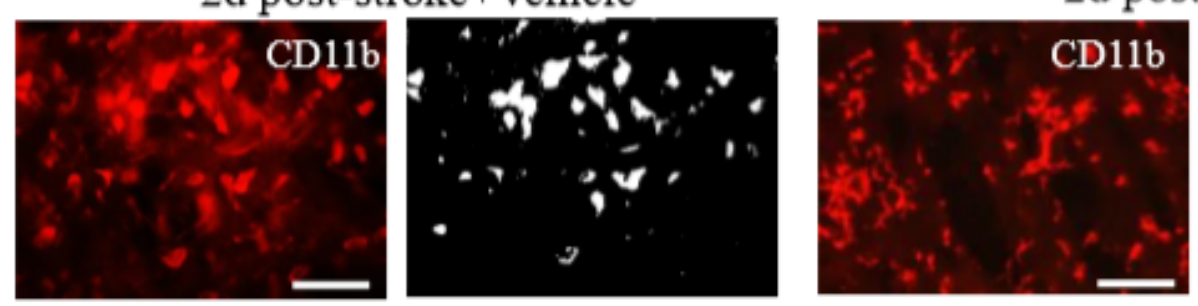

2 d post-stroke+VPA

E

$7 \mathrm{~d}$ post-stroke + Vehicle
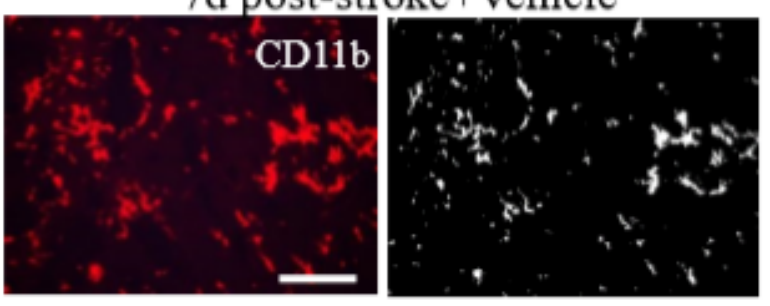

F
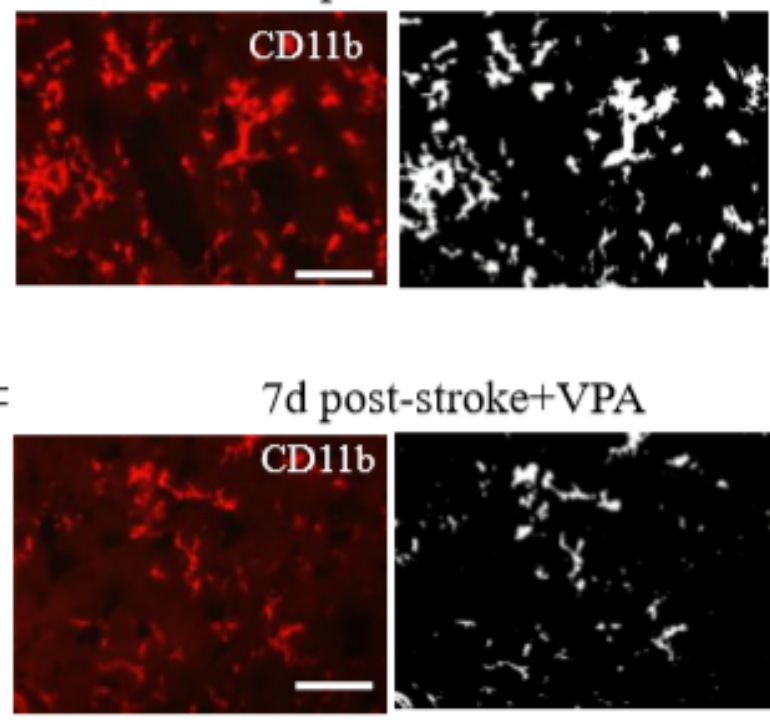

$7 \mathrm{~d}$ post-stroke+VPA

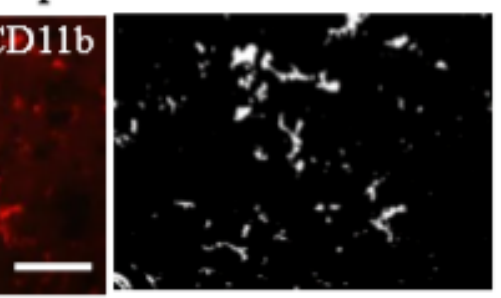

G

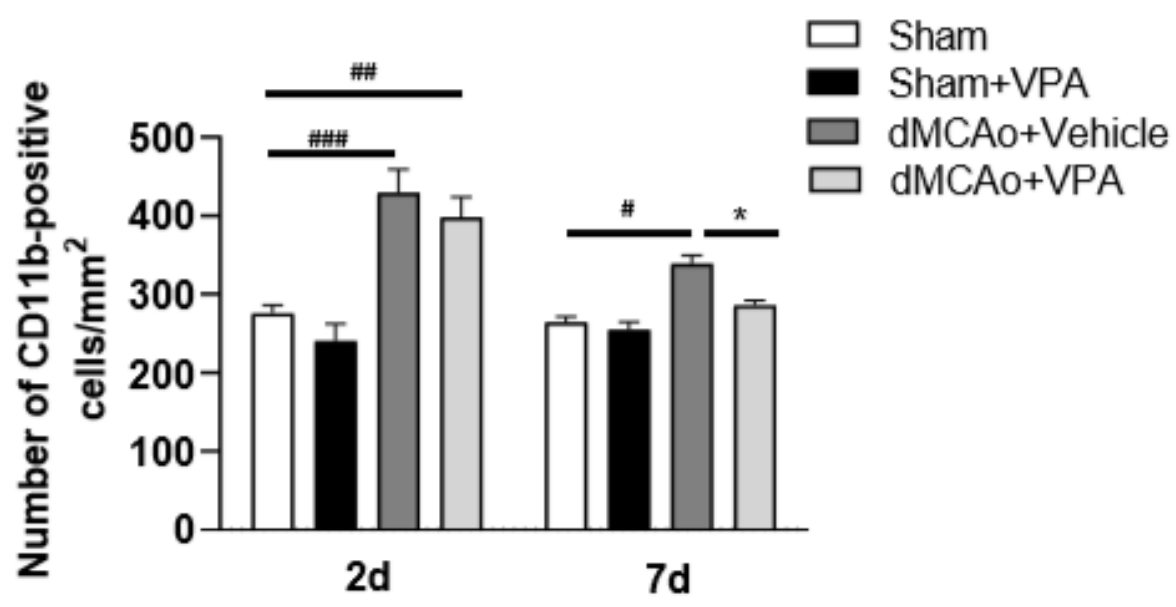

Figure 4

Post-stroke intraperitoneal injection of VPA decreases the number of CD11b-positive cells in the periinfarct cortex. A-F, representative photomicrographs of anti-CD11b immunofluorescent staining of the sham-operated cortex in vehicle-treated rats (A), sham-operated cortex in VPA-treated rats (B), peri-infarct cortex at 2 days after 90-minute dMCAo in vehicle-treated rats (C), peri-infarct cortex at 2 days after 90minute dMCAo in VPA-treated rats (D), peri-infarct cortex at 7 days after 90-minute dMCAo in vehicle- 
treated rats $(E)$, and peri-infarct cortex at 7 days after 90 -minute dMCAo in VPA-treated rats $(F)$. G, quantitation of CD11b-positive cells in the peri-infarct cortex at different time points showing accumulation of activated microglia/macrophages in the peri-infarct cortex at 2 and 7 days after ischemia. Original photomicrographs were subjected to a series of uniform Image $\mathrm{J}$ plugin protocols prior to conversion to binary images; binary images were then analyzed to calculate the number of CD11bpositive cells. $\# p<0.05, \# \# p<0.01$, and \#\#\# $p<0.001$ indicate statistical difference between peri-infarct cortex and sham-operated cortex at each time point, Bonferroni's multiple comparisons test, following one-way ANOVA. * $p<0.05$ indicates comparison with the dMCAo+ vehicle group with two-way ANOVA, Bonferroni's post hoc test. Five tissue sections per rat were used for the analysis $(n=8-10)$. Scale bar: 20 $\mu \mathrm{m}$. The data represent mean \pm SEM. 
A
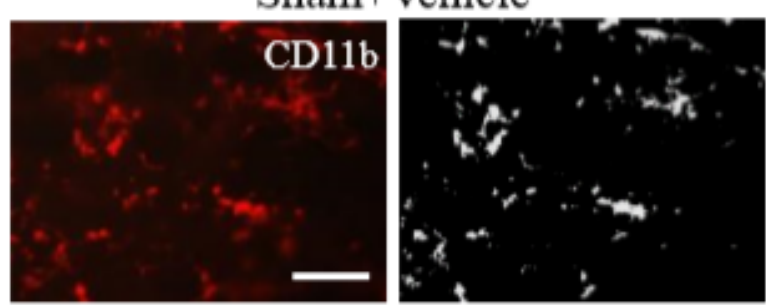

B

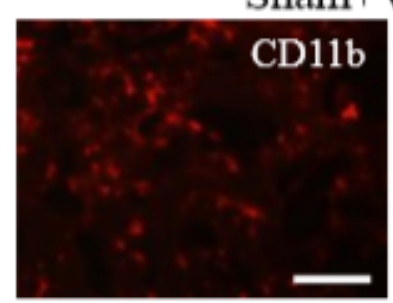

D

C
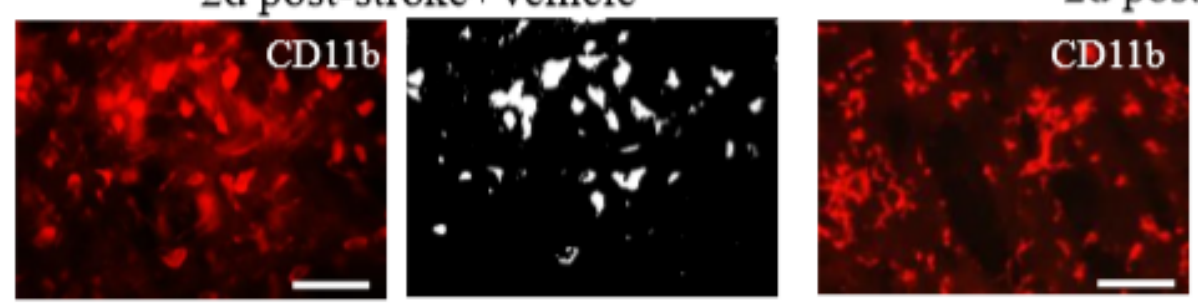

2 d post-stroke+VPA

E

$7 \mathrm{~d}$ post-stroke + Vehicle
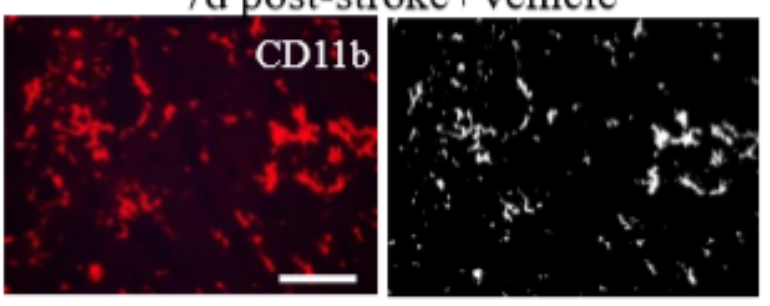

F
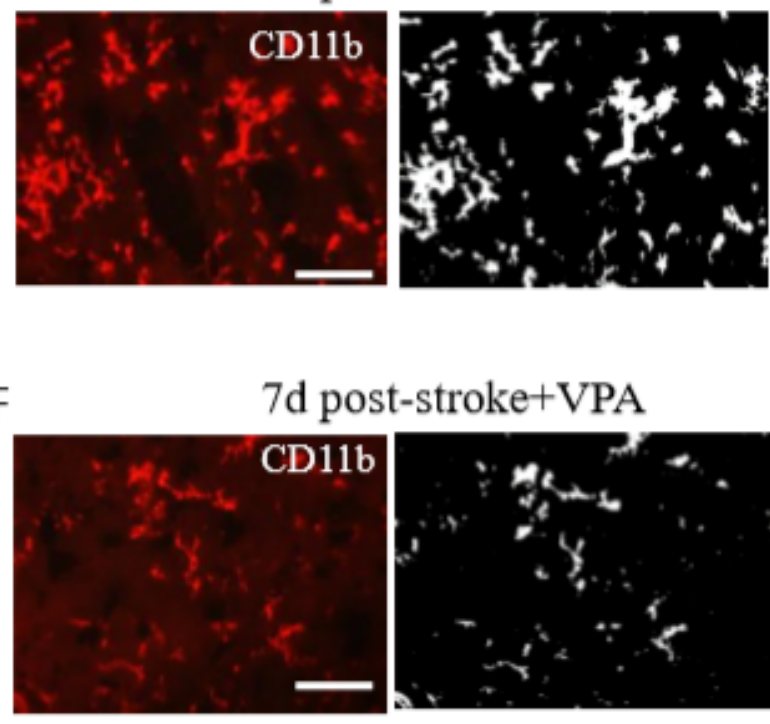

$7 \mathrm{~d}$ post-stroke+VPA

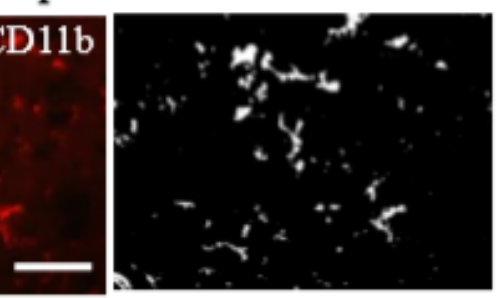

G

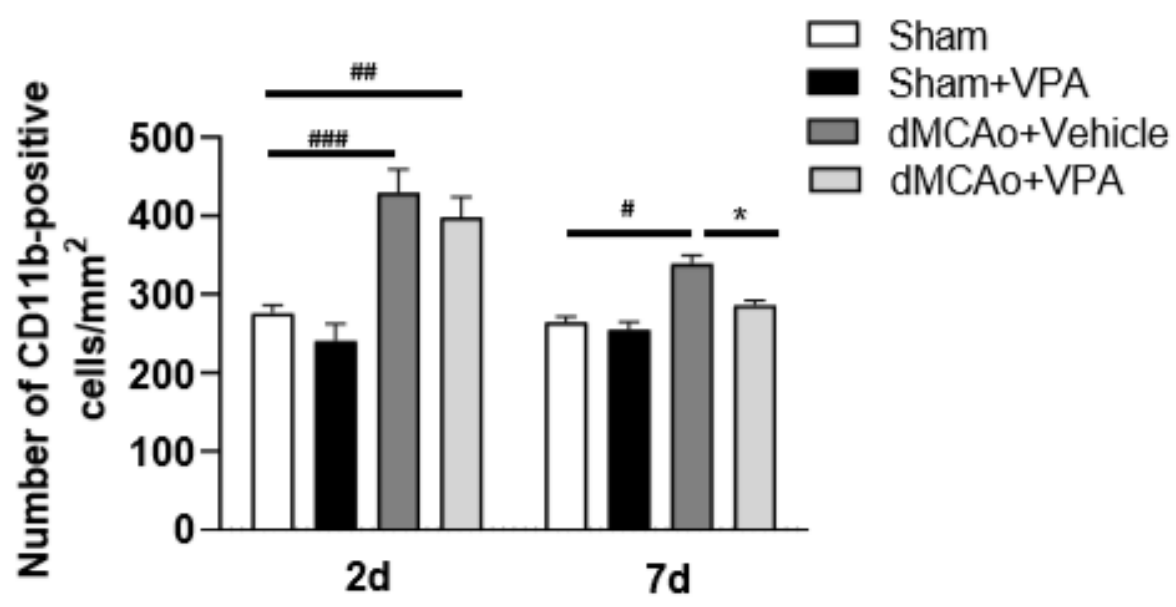

Figure 4

Post-stroke intraperitoneal injection of VPA decreases the number of CD11b-positive cells in the periinfarct cortex. A-F, representative photomicrographs of anti-CD11b immunofluorescent staining of the sham-operated cortex in vehicle-treated rats (A), sham-operated cortex in VPA-treated rats (B), peri-infarct cortex at 2 days after 90-minute dMCAo in vehicle-treated rats (C), peri-infarct cortex at 2 days after 90minute dMCAo in VPA-treated rats (D), peri-infarct cortex at 7 days after 90-minute dMCAo in vehicle- 
treated rats $(E)$, and peri-infarct cortex at 7 days after 90 -minute dMCAo in VPA-treated rats $(F)$. G, quantitation of CD11b-positive cells in the peri-infarct cortex at different time points showing accumulation of activated microglia/macrophages in the peri-infarct cortex at 2 and 7 days after ischemia. Original photomicrographs were subjected to a series of uniform Image $\mathrm{J}$ plugin protocols prior to conversion to binary images; binary images were then analyzed to calculate the number of CD11bpositive cells. $\# p<0.05, \# \# p<0.01$, and \#\#\# $p<0.001$ indicate statistical difference between peri-infarct cortex and sham-operated cortex at each time point, Bonferroni's multiple comparisons test, following one-way ANOVA. * $p<0.05$ indicates comparison with the dMCAo+ vehicle group with two-way ANOVA, Bonferroni's post hoc test. Five tissue sections per rat were used for the analysis $(n=8-10)$. Scale bar: 20 $\mu \mathrm{m}$. The data represent mean \pm SEM. 
A

Ramified/Branched

\section{Unramified/Ameodboid}

B
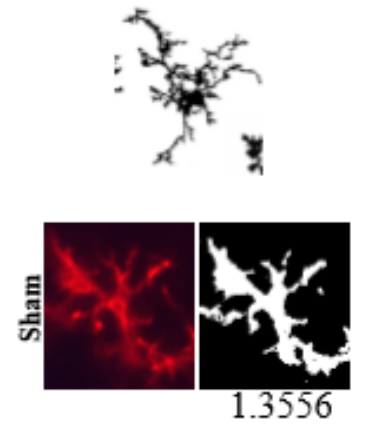

C

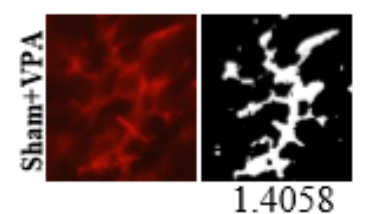

D

$$
\text { 竞 }
$$

E

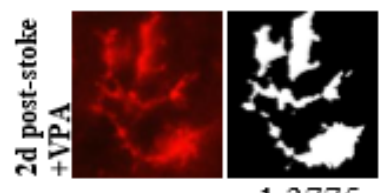

F

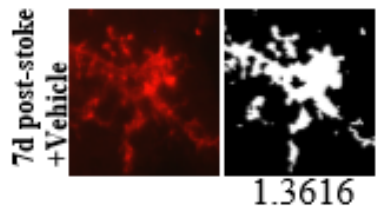

G

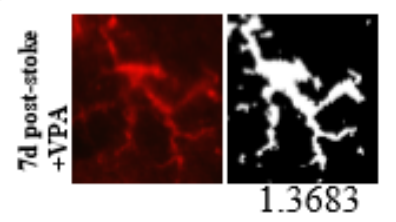

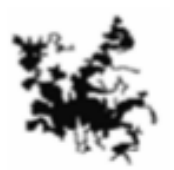
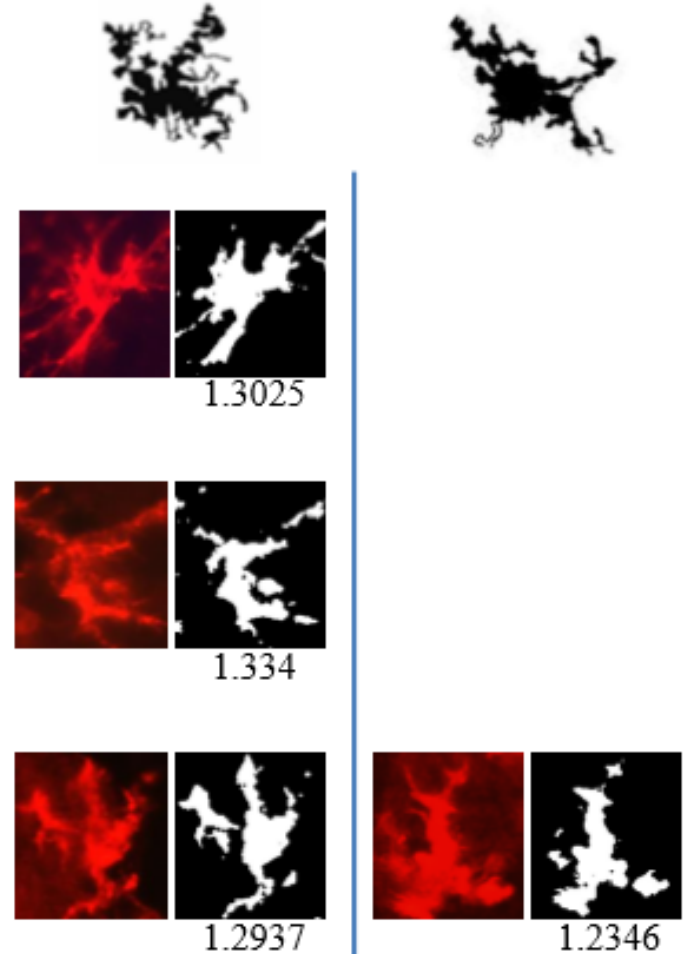

1.2346

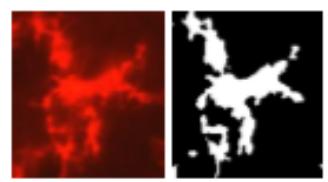

1.2745
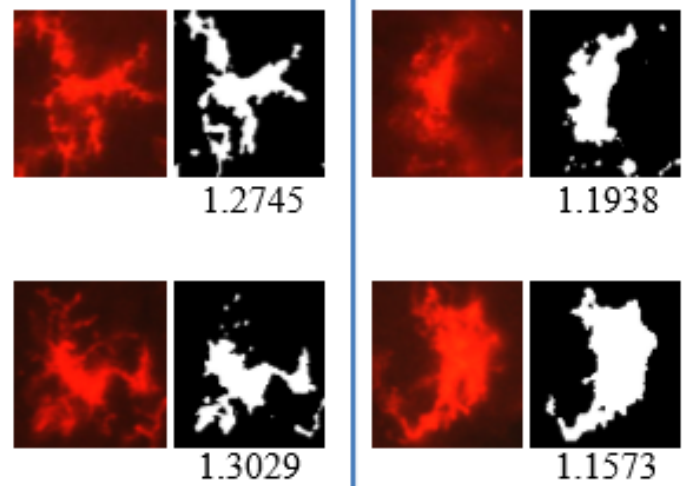

1.1938

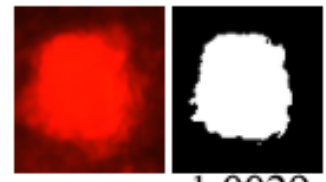

1.0029
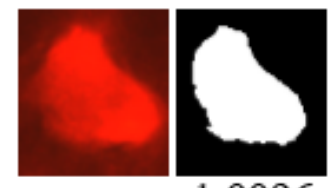

1.0096

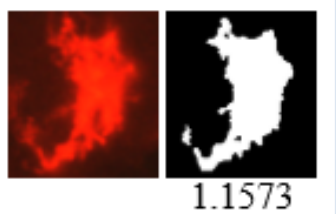

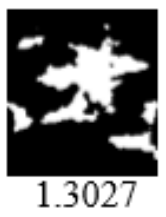

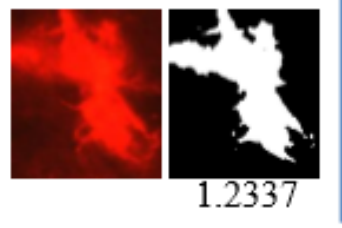

\section{Figure 5}

Complexity analysis of CD11b-positive cell morphologies in the peri-infarct cortex with/without VPA administration. A, microglia/macrophages are morphologically dynamic cells able to change form from highly ramified to a completely amoeboid-like shape lacking processes. This transition can be very rapid under pathological conditions. The forms illustrated here represent snapshots of a transformation that is reversible at every time point, with variation with each form shown. B-G, complexity analysis of 
microglia/macrophages in CD11b-stained tissue. The process to prepare photomicrographs for complexity analysis: Original photomicrographs were subjected to a series of uniform ImageJ plugin protocols prior to conversion to binary images. Binary images were then analyzed by using FracLac for ImageJ, which quantifies single cell complexity (fractal dimension, Df). The calculated Df of the cell is shown below its binary image. Representative images of morphological changes in CD11b-staining cells within the sham-operated cortex in vehicle-treated rats (B), sham-operated cortex in VPA-treated rats (C), peri-infarct cortex at 2 days after 90 -minute dMCAo in vehicle-treated rats (D), peri-infarct cortex at 2 days after 90-minute dMCAo in VPA-treated rats $(E)$, peri-infarct cortex at 7 days after 90-minute dMCAo in vehicle-treated rats $(F)$, and peri-infarct at 7 days after 90-minute dMCAo in VPA-treated rats (G). 
A

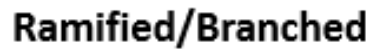

\section{Unramified/Ameodboid}

B
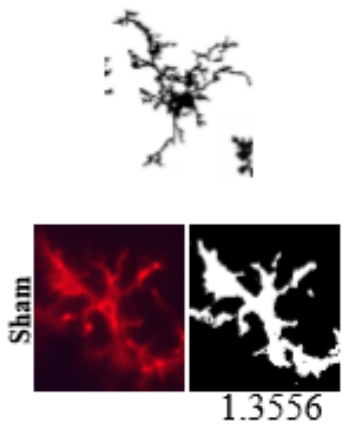

C

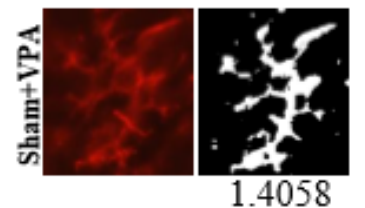

D

$$
\text { 总 }
$$

E

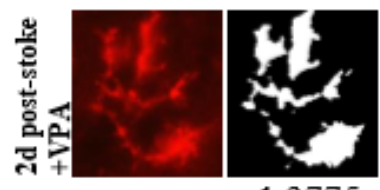

F

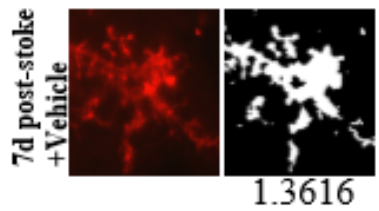

G

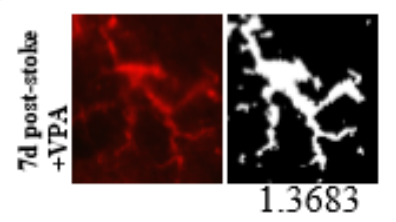

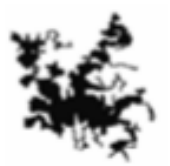
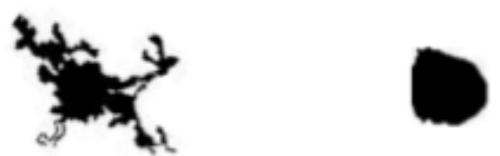
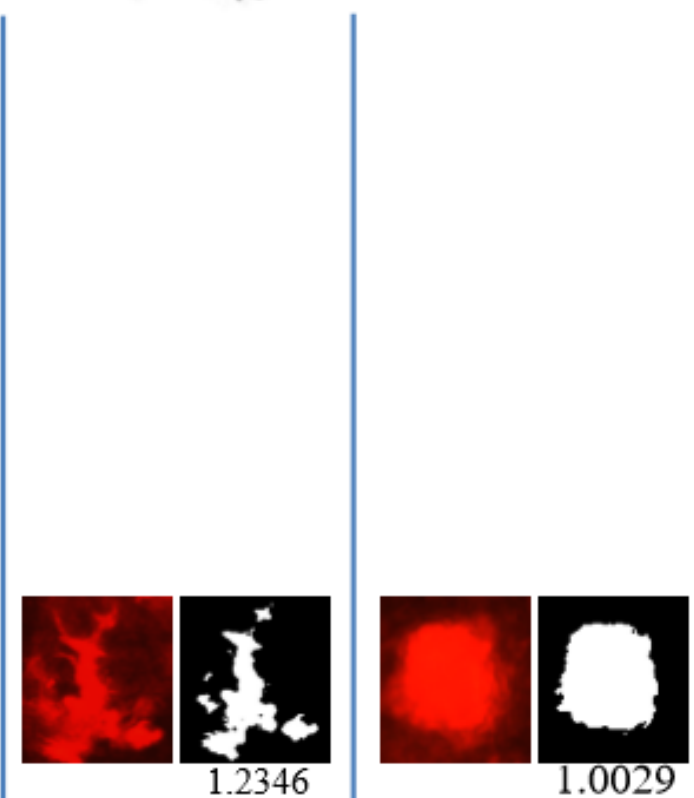

1.0029
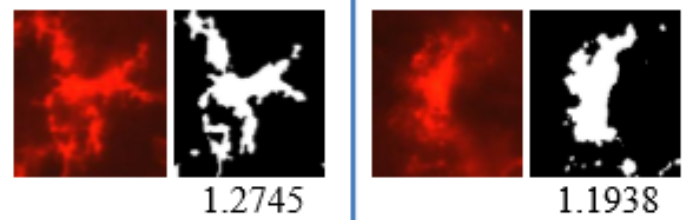

1.1938
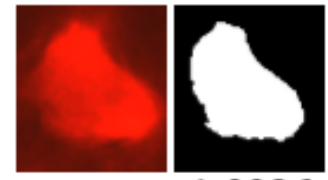

1.0096

Figure 5

Complexity analysis of CD11b-positive cell morphologies in the peri-infarct cortex with/without VPA administration. A, microglia/macrophages are morphologically dynamic cells able to change form from highly ramified to a completely amoeboid-like shape lacking processes. This transition can be very rapid under pathological conditions. The forms illustrated here represent snapshots of a transformation that is reversible at every time point, with variation with each form shown. B-G, complexity analysis of 
microglia/macrophages in CD11b-stained tissue. The process to prepare photomicrographs for complexity analysis: Original photomicrographs were subjected to a series of uniform ImageJ plugin protocols prior to conversion to binary images. Binary images were then analyzed by using FracLac for ImageJ, which quantifies single cell complexity (fractal dimension, Df). The calculated Df of the cell is shown below its binary image. Representative images of morphological changes in CD11b-staining cells within the sham-operated cortex in vehicle-treated rats (B), sham-operated cortex in VPA-treated rats (C), peri-infarct cortex at 2 days after 90 -minute dMCAo in vehicle-treated rats (D), peri-infarct cortex at 2 days after 90-minute dMCAo in VPA-treated rats $(E)$, peri-infarct cortex at 7 days after 90-minute dMCAo in vehicle-treated rats $(F)$, and peri-infarct at 7 days after 90-minute dMCAo in VPA-treated rats (G). 
A

Ramified/Branched

\section{Unramified/Ameodboid}

B
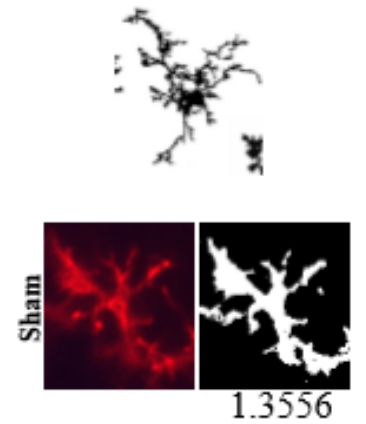

C

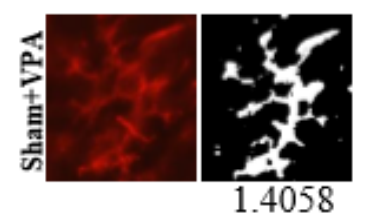

D

$$
\text { 竞 }
$$

E

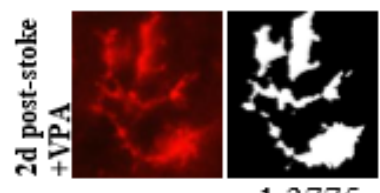

F

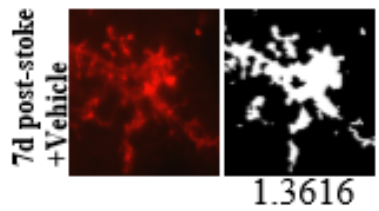

G

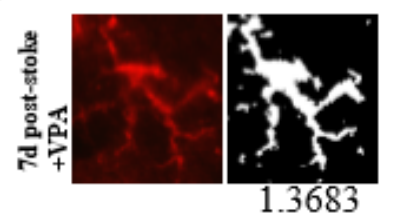

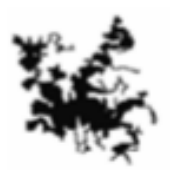
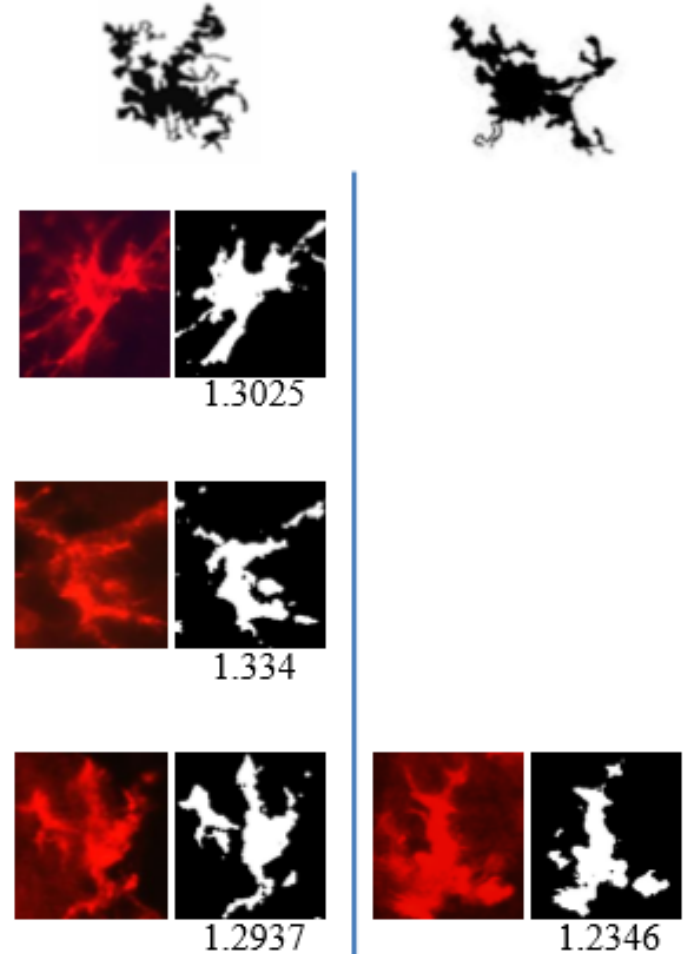

1.2346

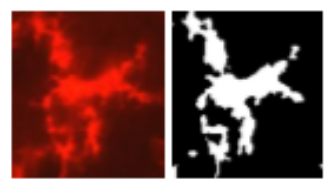

1.2745
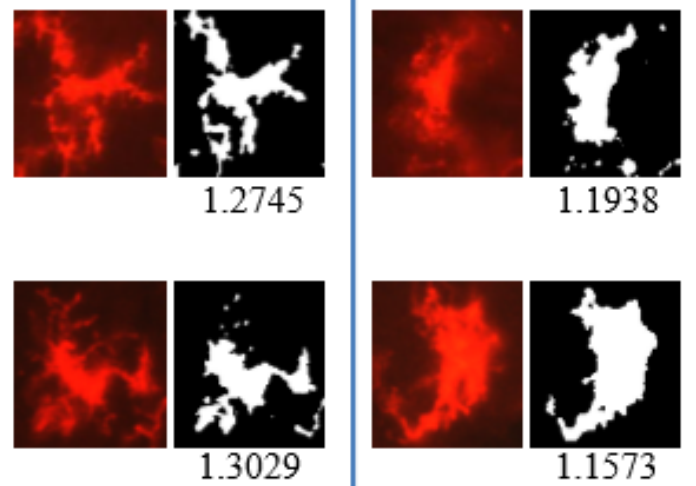

1.1938

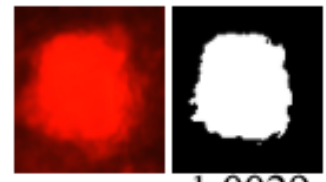

1.0029
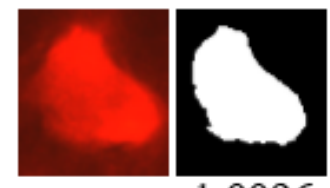

1.0096

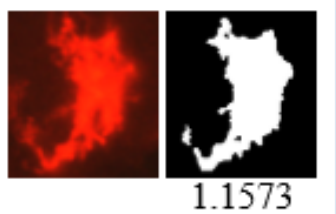

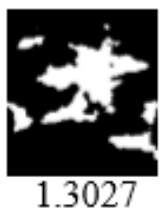

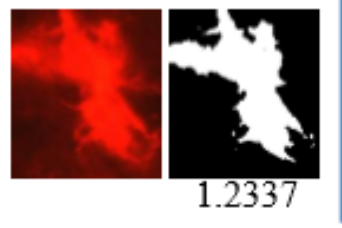

\section{Figure 5}

Complexity analysis of CD11b-positive cell morphologies in the peri-infarct cortex with/without VPA administration. A, microglia/macrophages are morphologically dynamic cells able to change form from highly ramified to a completely amoeboid-like shape lacking processes. This transition can be very rapid under pathological conditions. The forms illustrated here represent snapshots of a transformation that is reversible at every time point, with variation with each form shown. B-G, complexity analysis of 
microglia/macrophages in CD11b-stained tissue. The process to prepare photomicrographs for complexity analysis: Original photomicrographs were subjected to a series of uniform ImageJ plugin protocols prior to conversion to binary images. Binary images were then analyzed by using FracLac for ImageJ, which quantifies single cell complexity (fractal dimension, Df). The calculated Df of the cell is shown below its binary image. Representative images of morphological changes in CD11b-staining cells within the sham-operated cortex in vehicle-treated rats (B), sham-operated cortex in VPA-treated rats (C), peri-infarct cortex at 2 days after 90-minute dMCAo in vehicle-treated rats (D), peri-infarct cortex at 2 days after 90-minute dMCAo in VPA-treated rats $(E)$, peri-infarct cortex at 7 days after 90-minute dMCAo in vehicle-treated rats $(F)$, and peri-infarct at 7 days after 90-minute dMCAo in VPA-treated rats $(G)$.

A

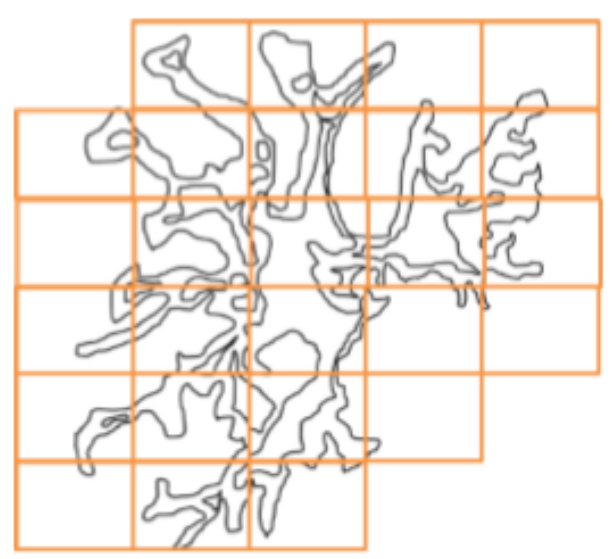

$\mathrm{C}$

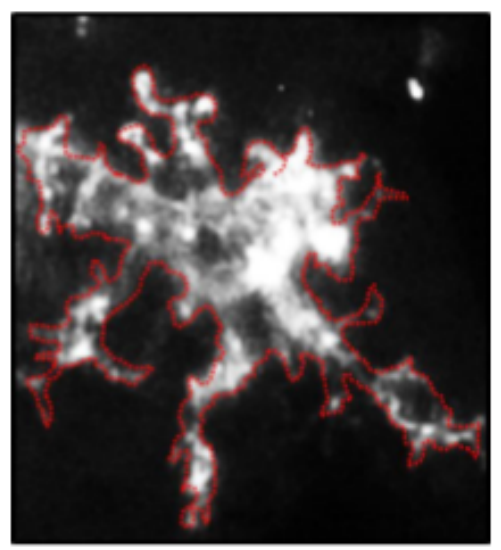

B
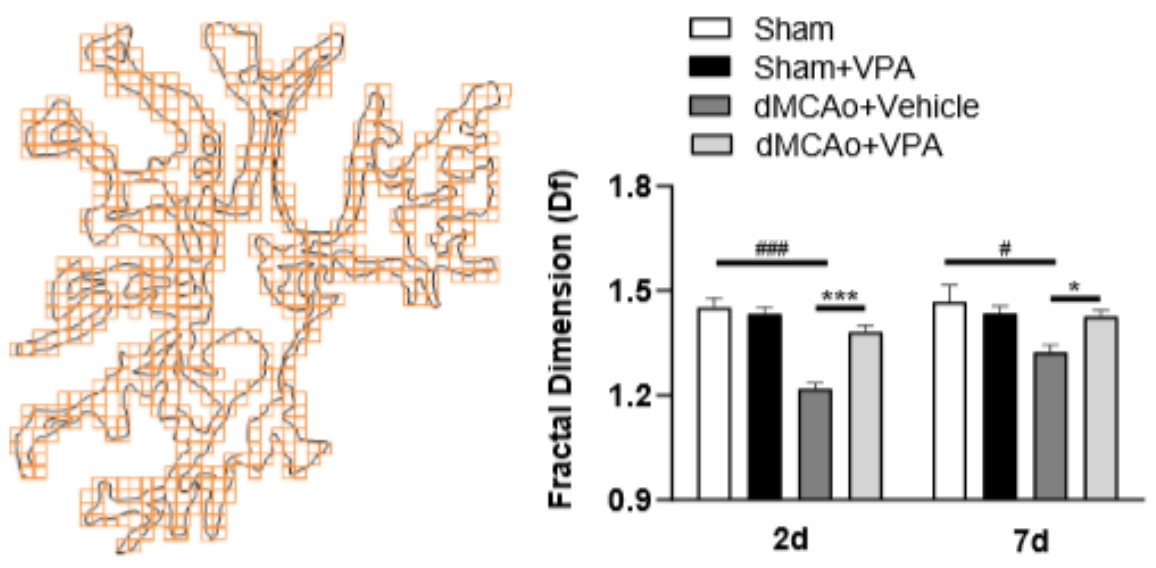

D

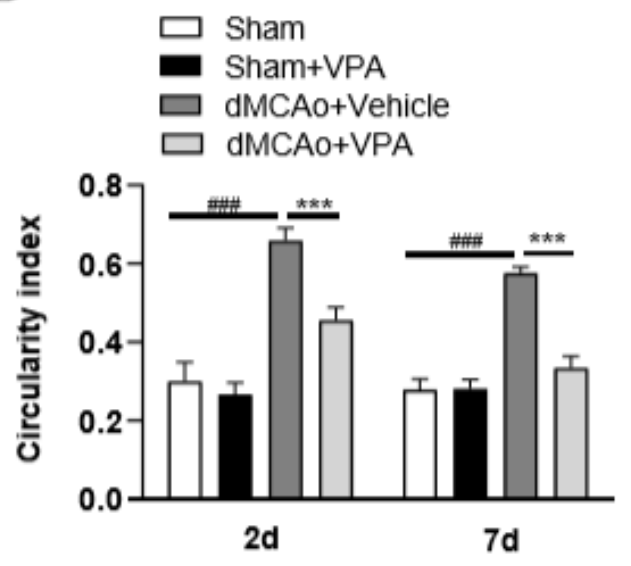

Figure 6

Effects of VPA treatment on responses of CD11b-positive cells after ischemic cortical stroke. (A), illustration of FracLac box counting method to derive fractal dimension calculations of a CD11b-positive cell outline. Shape detail is quantified as scale increase, represented by yellow boxes. Box counting equation is summarized in Table 1. (B), Summary data and statistical analysis of fractal dimension at 2 days and 7 days after ischemia. Fractal dimension was decreased in the peri-infarct cortex compared 
with the sham-operated cortex at 2 days and 7 days post-stroke. However, VPA treatment restored the fractal dimension of CD11b-positive cells in the peri-infarct cortex at 2 days and 7 days post-stroke. All post-hoc analyses are reported in the figure ( $2 \mathrm{~d}$ : \#\#\# $\mathrm{p}<0.001 \mathrm{vs}$. sham and dMCAo+vehicle; $* \star \star ~ p<$ 0.001 vs. dMCAo+vehicle and dMCAo+VPA; 7d: \# $p<0.05$ vs. sham and dMCAo+vehicle; ${ }^{*} p<0.05$ vs.

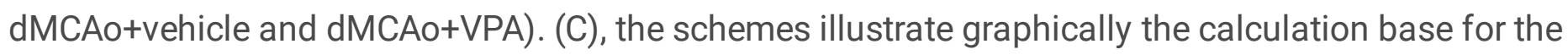
circularity index for the manual analysis. (D), circularity index was increased in the peri-infarct cortex compared with the sham-operated cortex at 2 days and 7 days post-stroke. However, VPA treatment decreased the circularity index of CD11b-positive cells in the peri-infarct cortex at 2 days and 7 days poststroke. All post-hoc analyses are reported in the figure ( $2 \mathrm{~d}$ : \#\#\# $\mathrm{p}<0.001 \mathrm{vs}$. sham and dMCAo+vehicle; *** $p<0.001$ vs. dMCAo+vehicle and dMCAo+VPA; 7d: \#\#\# $p<0.001$ vs. sham and dMCAo+vehicle; $* \star *$ $p<0.001$ vs. $d M C A o+v e h i c l e$ and dMCAo+VPA). Twenty-five to thirty cells per region of $n=4$ rats in each group.

A

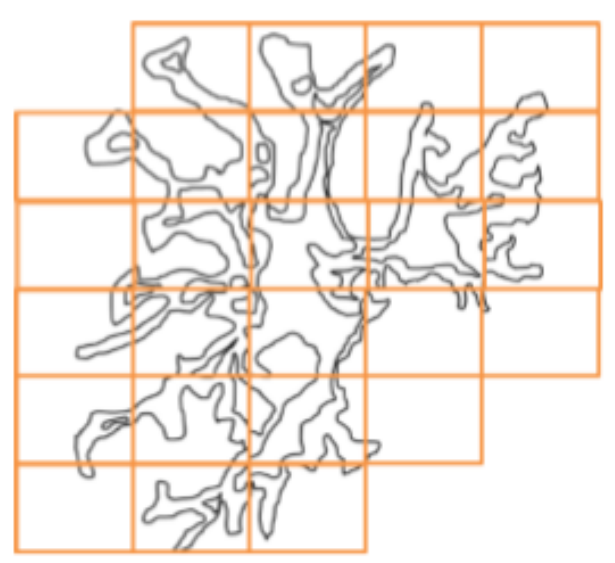

$\mathrm{C}$

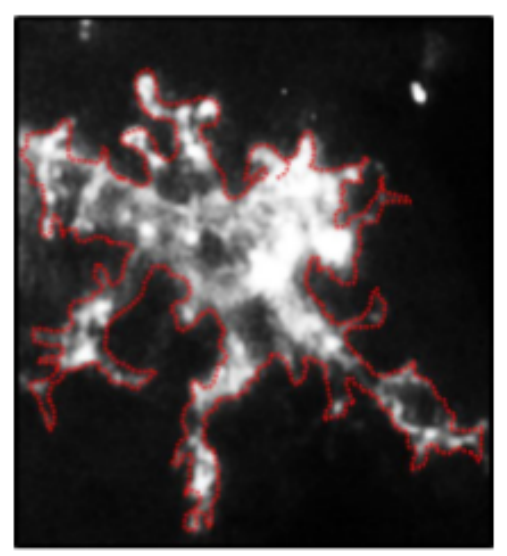

B

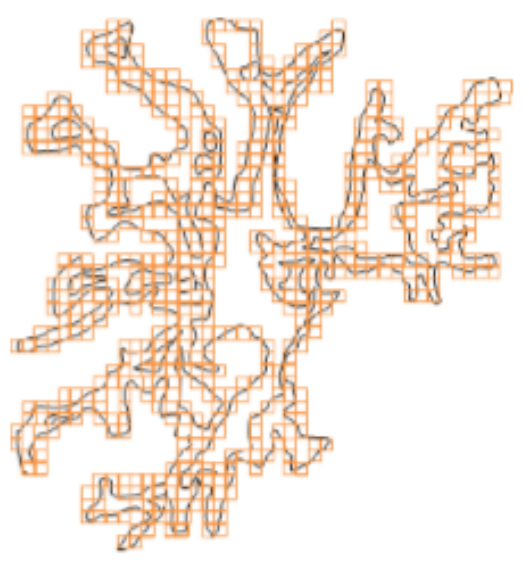

D

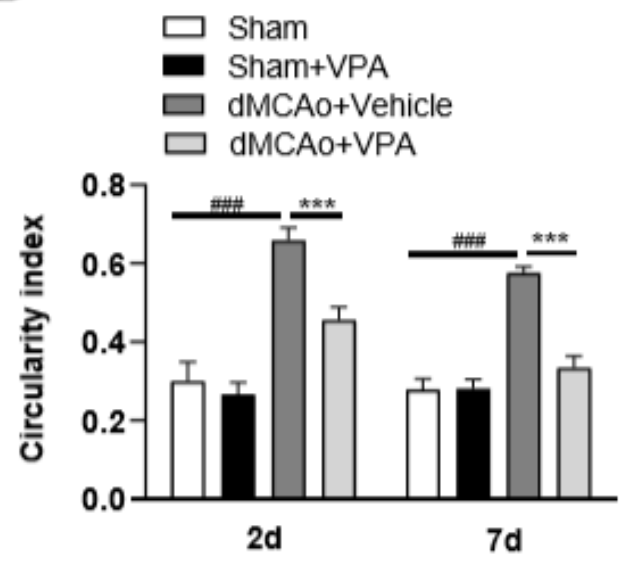

\section{Figure 6}

Effects of VPA treatment on responses of CD11b-positive cells after ischemic cortical stroke. (A), illustration of FracLac box counting method to derive fractal dimension calculations of a CD11b-positive 
cell outline. Shape detail is quantified as scale increase, represented by yellow boxes. Box counting equation is summarized in Table 1. (B), Summary data and statistical analysis of fractal dimension at 2 days and 7 days after ischemia. Fractal dimension was decreased in the peri-infarct cortex compared with the sham-operated cortex at 2 days and 7 days post-stroke. However, VPA treatment restored the fractal dimension of CD11b-positive cells in the peri-infarct cortex at 2 days and 7 days post-stroke. All post-hoc analyses are reported in the figure ( $2 \mathrm{~d}$ : \#\#\# $\mathrm{p}<0.001 \mathrm{vs}$. sham and dMCAo+vehicle; $* \star \star \mathrm{p}<$ 0.001 vs. dMCAo+vehicle and dMCAo+VPA; 7d: \# $p<0.05$ vs. sham and dMCAo+vehicle; ${ }^{*} p<0.05$ vs. $d M C A o+v e h i c l e$ and $d M C A o+V P A)$. (C), the schemes illustrate graphically the calculation base for the circularity index for the manual analysis. (D), circularity index was increased in the peri-infarct cortex compared with the sham-operated cortex at 2 days and 7 days post-stroke. However, VPA treatment decreased the circularity index of CD11b-positive cells in the peri-infarct cortex at 2 days and 7 days poststroke. All post-hoc analyses are reported in the figure ( $2 \mathrm{~d}$ : \#\#\# $p<0.001$ vs. sham and dMCAo+vehicle; $\star \star \star ~ p<0.001$ vs. dMCAo+vehicle and dMCAo+VPA; 7d: \#\#\# $p<0.001$ vs. sham and dMCAo+vehicle; $* \star *$ $p<0.001$ vs. dMCAo+vehicle and dMCAo+VPA). Twenty-five to thirty cells per region of $n=4$ rats in each group.

A

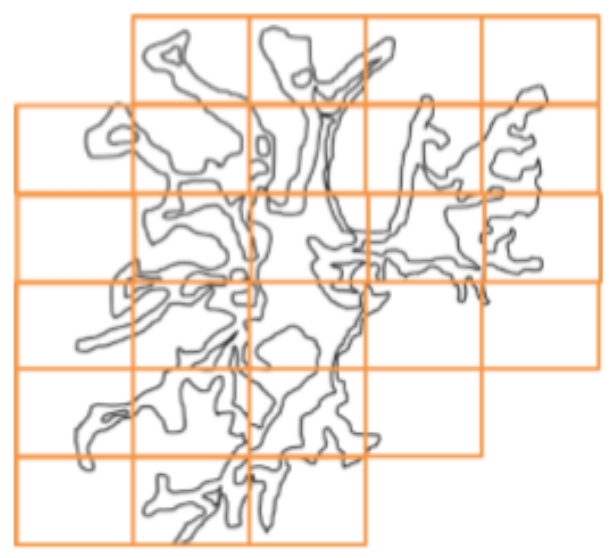

$\mathrm{C}$

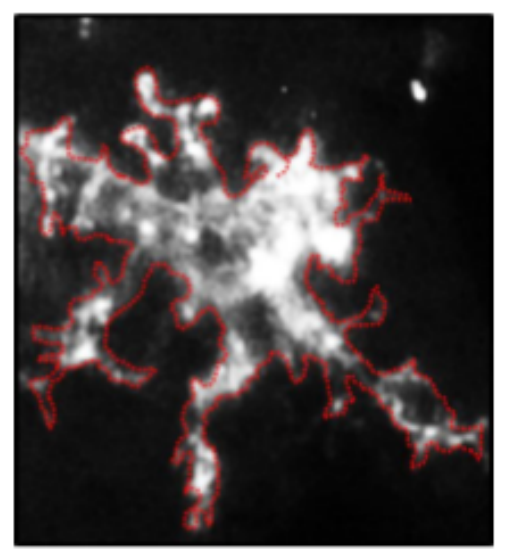

$\mathrm{B}$

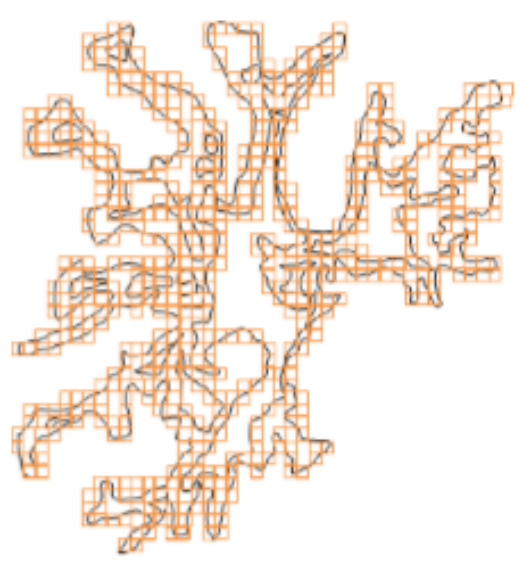

D

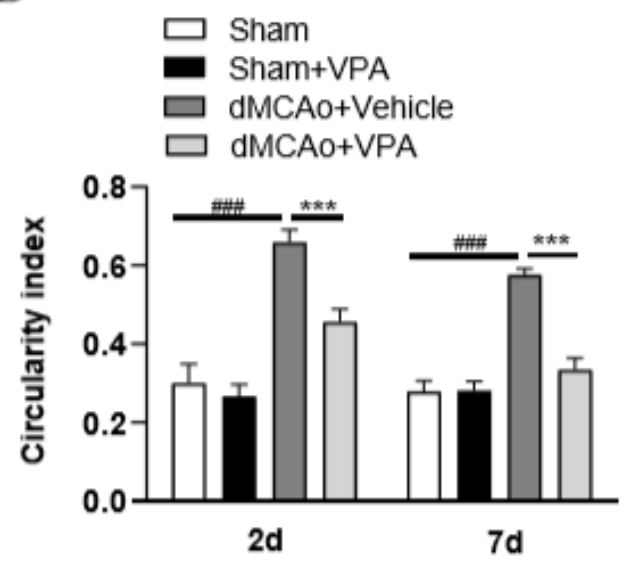


Effects of VPA treatment on responses of CD11b-positive cells after ischemic cortical stroke. (A), illustration of FracLac box counting method to derive fractal dimension calculations of a CD11b-positive cell outline. Shape detail is quantified as scale increase, represented by yellow boxes. Box counting equation is summarized in Table 1. (B), Summary data and statistical analysis of fractal dimension at 2 days and 7 days after ischemia. Fractal dimension was decreased in the peri-infarct cortex compared with the sham-operated cortex at 2 days and 7 days post-stroke. However, VPA treatment restored the fractal dimension of CD11b-positive cells in the peri-infarct cortex at 2 days and 7 days post-stroke. All post-hoc analyses are reported in the figure $(2 \mathrm{~d}$ : \#\#\# $\mathrm{p}<0.001 \mathrm{vs}$. sham and dMCAo+vehicle; $* \star \star p<$ 0.001 vs. dMCAo+vehicle and dMCAo+VPA; 7d: \# $p<0.05$ vs. sham and dMCAo+vehicle; * $p<0.05$ vs. $\mathrm{dMCAo+vehicle} \mathrm{and} \mathrm{dMCAo+VPA).(C),} \mathrm{the} \mathrm{schemes} \mathrm{illustrate} \mathrm{graphically} \mathrm{the} \mathrm{calculation} \mathrm{base} \mathrm{for} \mathrm{the}$ circularity index for the manual analysis. (D), circularity index was increased in the peri-infarct cortex compared with the sham-operated cortex at 2 days and 7 days post-stroke. However, VPA treatment decreased the circularity index of CD11b-positive cells in the peri-infarct cortex at 2 days and 7 days poststroke. All post-hoc analyses are reported in the figure ( $2 \mathrm{~d}$ : \#\#\# $\mathrm{p}<0.001$ vs. sham and dMCAo+vehicle; $\star \star \star ~ p<0.001$ vs. dMCAo+vehicle and dMCAo+VPA; 7d: \#\#\# $p<0.001$ vs. sham and dMCAo+vehicle; *** $p<0.001$ vs. dMCAo+vehicle and dMCAo+VPA). Twenty-five to thirty cells per region of $n=4$ rats in each group. 
A

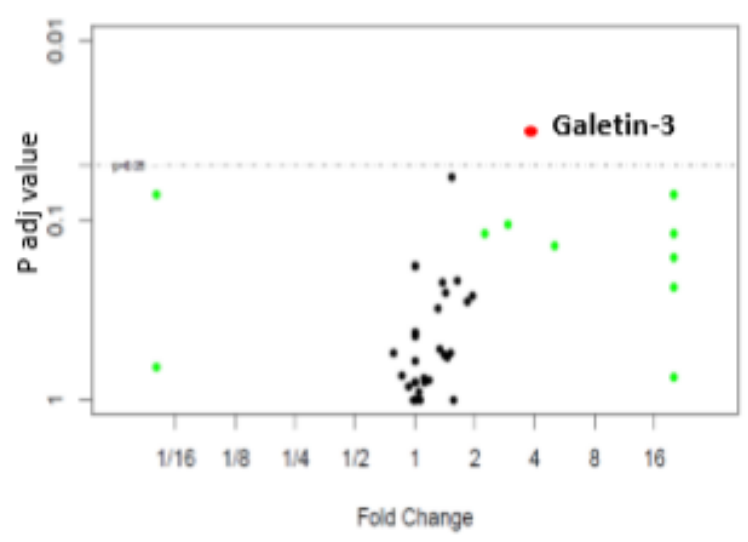

$\mathrm{C}$

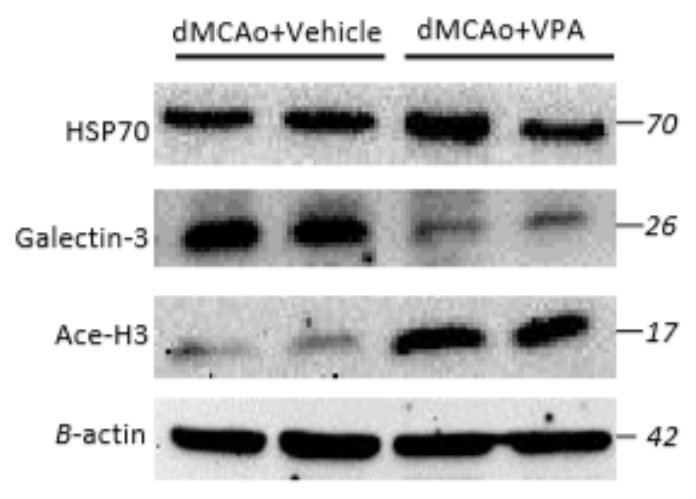

$\mathrm{E}$

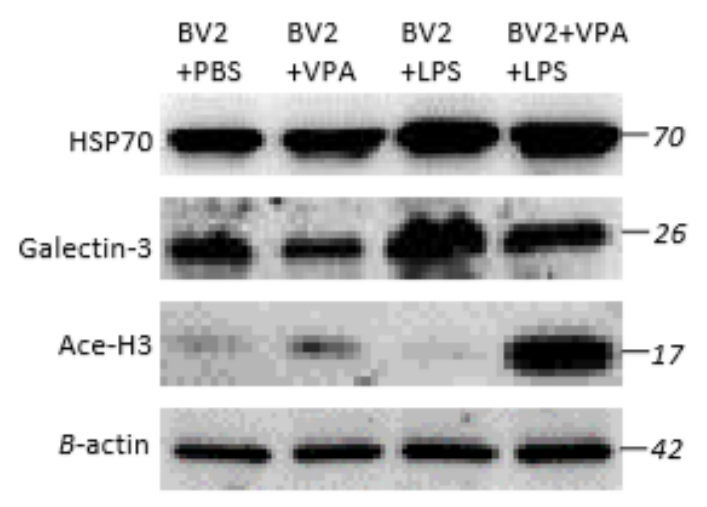

B

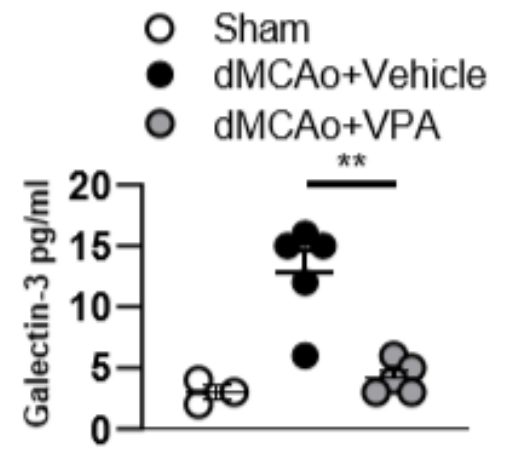

$\mathrm{D}$

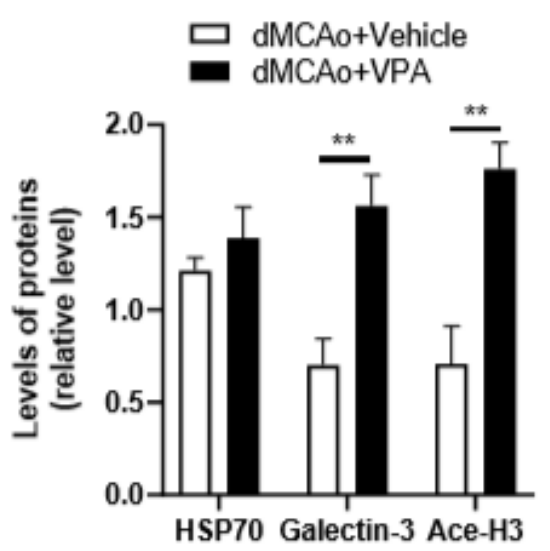

F

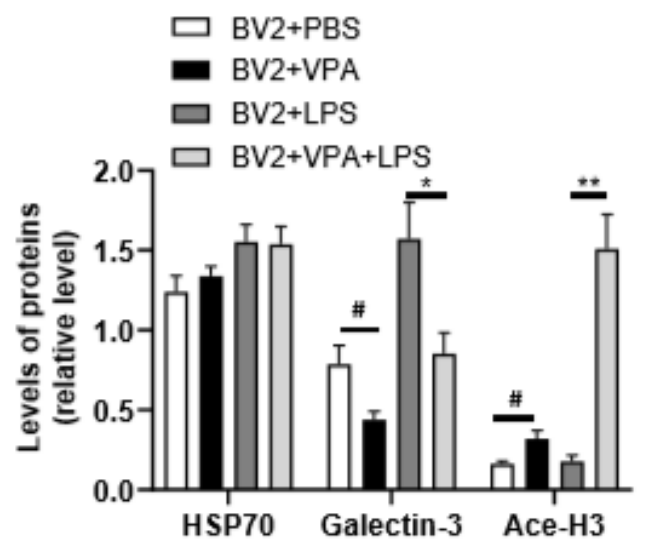

\section{Figure 7}

Effects of VPA treatment on galectin-3 expression in the peri-infarct cortex and BV2 cells. (A), volcano plot comparing the fold changes and adjusted p-values of 67 biomarkers in which each point represents a biomarker. (B), VPA suppresses galectin-3 production in the peri-infarct cortex of rats with ischemic brain injury. Galectin-3 concentrations in the peri-infarct cortex at 2 days after dMCAo surgery were measured by ELISA $(n=5) .{ }^{* *} p<0.01$, compared with the vehicle-treated group. (C), protein levels of HSP70, 
acetylated $\mathrm{H} 3$ (Ace-H3), galectin-3, and $\beta$-actin were determined by western blot analysis. (D), quantified results of HSP70, Ace-H3, and galectin-3. Each column and vertical bar represents the mean \pm SEM of 5 animals. ** $P<0.01$, Student's t-test. (E), BV2 cells were activated using LPS $(1 \mu \mathrm{g} / \mathrm{ml})$ and, simultaneously, in the presence or absence of VPA as indicated. After 6 hours of treatment, the protein levels of HSP70, acetylated H3 (Ace-H3), galectin-3, and $\beta$-actin were determined by western blot analysis. (F), VPA treatment enhances the levels of Ace-H3, but suppresses galectin-3 production in the BV2 cells with/without LPS exposure. \# $p<0.05$ vs. control. ${ }^{*} p<0.05$ and ${ }^{* *} p<0.01$ indicate comparison with LPStreated BV2 cells with Student's t-test. The data represent mean \pm SEM. 
A

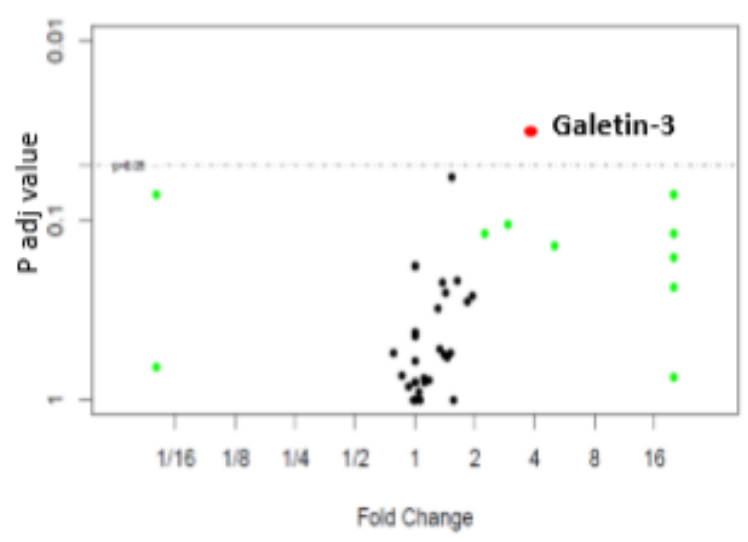

$\mathrm{C}$

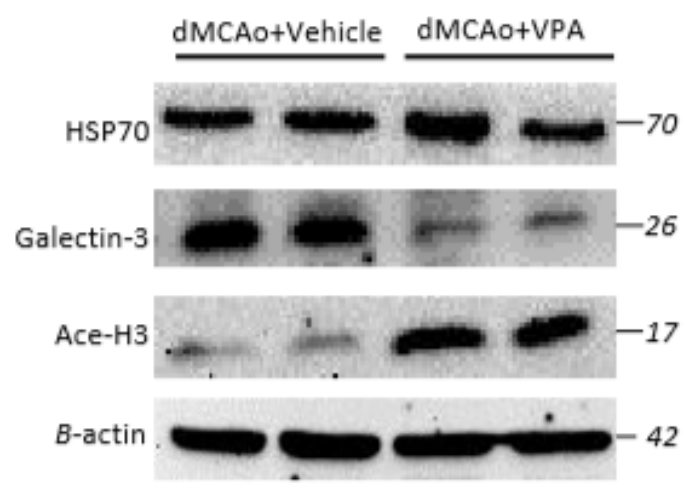

$\mathrm{E}$

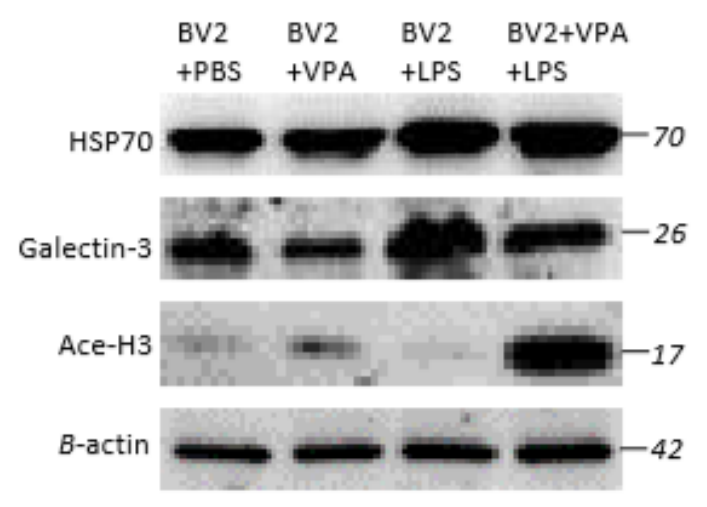

B

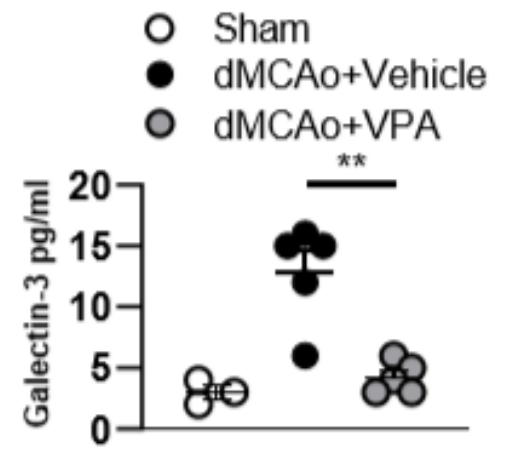

$\mathrm{D}$

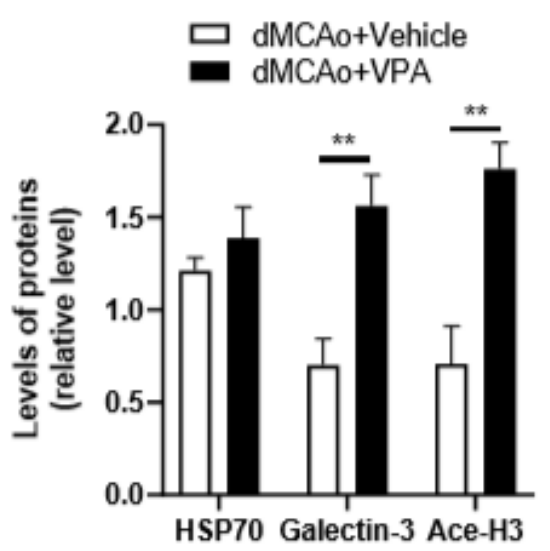

F

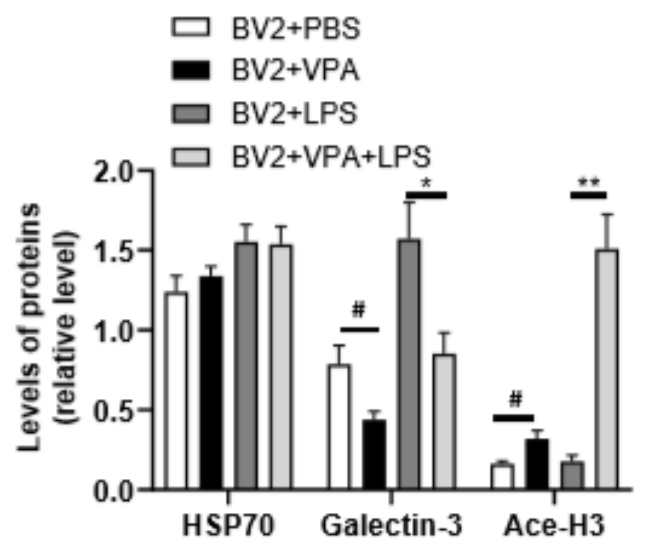

\section{Figure 7}

Effects of VPA treatment on galectin-3 expression in the peri-infarct cortex and BV2 cells. (A), volcano plot comparing the fold changes and adjusted p-values of 67 biomarkers in which each point represents a biomarker. (B), VPA suppresses galectin-3 production in the peri-infarct cortex of rats with ischemic brain injury. Galectin-3 concentrations in the peri-infarct cortex at 2 days after dMCAo surgery were measured by ELISA $(n=5) .{ }^{* *} p<0.01$, compared with the vehicle-treated group. (C), protein levels of HSP70, 
acetylated $\mathrm{H} 3$ (Ace-H3), galectin-3, and $\beta$-actin were determined by western blot analysis. (D), quantified results of HSP70, Ace-H3, and galectin-3. Each column and vertical bar represents the mean \pm SEM of 5 animals. ** $P<0.01$, Student's t-test. (E), BV2 cells were activated using LPS $(1 \mu \mathrm{g} / \mathrm{ml})$ and, simultaneously, in the presence or absence of VPA as indicated. After 6 hours of treatment, the protein levels of HSP70, acetylated H3 (Ace-H3), galectin-3, and $\beta$-actin were determined by western blot analysis. (F), VPA treatment enhances the levels of Ace-H3, but suppresses galectin-3 production in the BV2 cells with/without LPS exposure. \# $p<0.05$ vs. control. ${ }^{*} p<0.05$ and ${ }^{* *} p<0.01$ indicate comparison with LPStreated BV2 cells with Student's t-test. The data represent mean \pm SEM. 
A

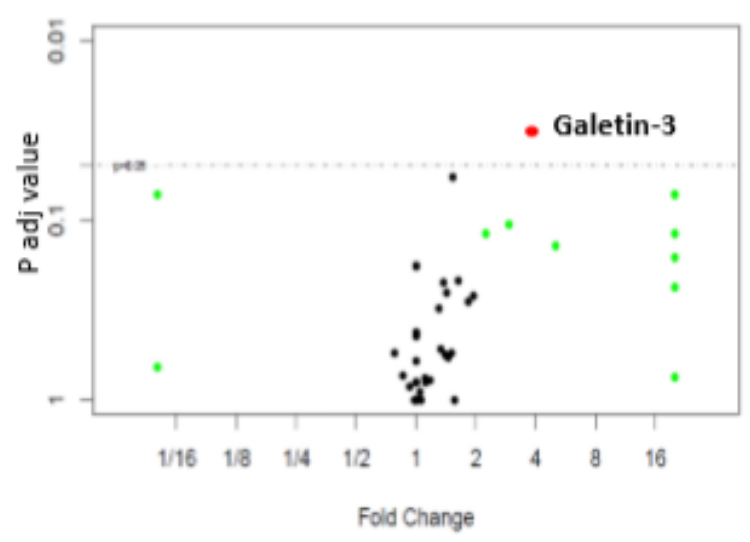

$\mathrm{C}$

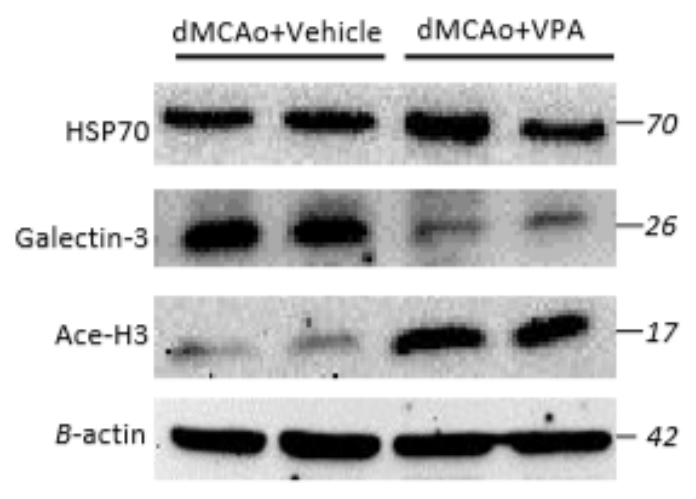

$\mathrm{E}$

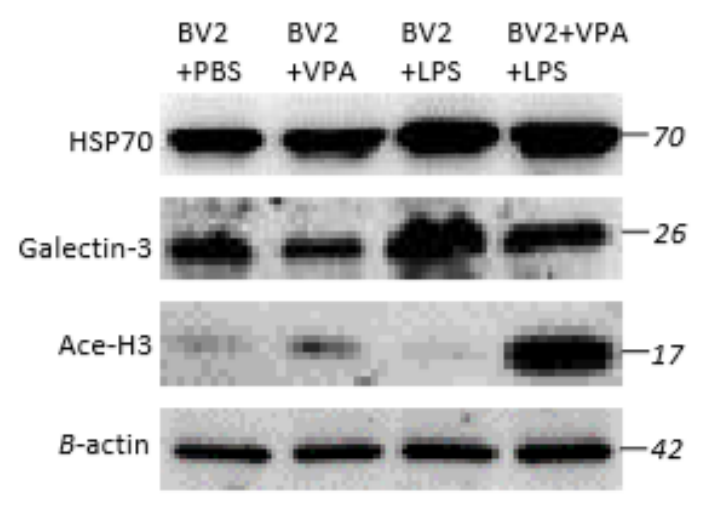

B

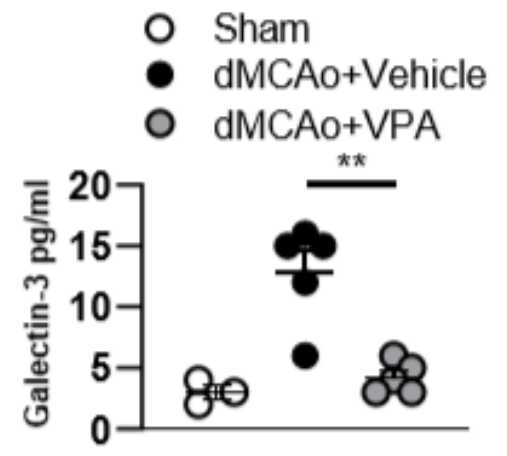

$\mathrm{D}$

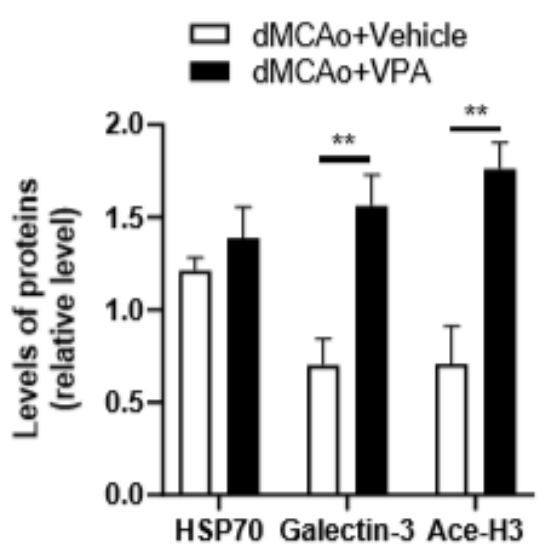

F

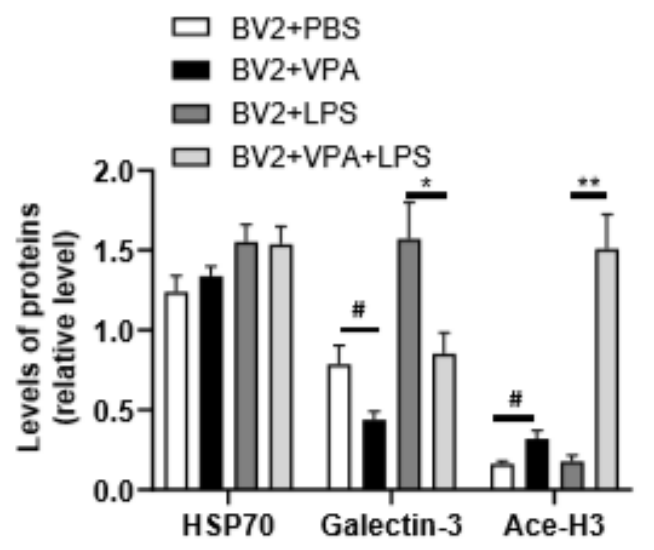

\section{Figure 7}

Effects of VPA treatment on galectin-3 expression in the peri-infarct cortex and BV2 cells. (A), volcano plot comparing the fold changes and adjusted p-values of 67 biomarkers in which each point represents a biomarker. (B), VPA suppresses galectin-3 production in the peri-infarct cortex of rats with ischemic brain injury. Galectin-3 concentrations in the peri-infarct cortex at 2 days after dMCAo surgery were measured by ELISA $(n=5) .{ }^{* *} p<0.01$, compared with the vehicle-treated group. (C), protein levels of HSP70, 
acetylated $\mathrm{H} 3$ (Ace-H3), galectin-3, and $\beta$-actin were determined by western blot analysis. (D), quantified results of HSP70, Ace-H3, and galectin-3. Each column and vertical bar represents the mean \pm SEM of 5 animals. ** $P<0.01$, Student's t-test. (E), BV2 cells were activated using LPS $(1 \mu \mathrm{g} / \mathrm{ml})$ and, simultaneously, in the presence or absence of VPA as indicated. After 6 hours of treatment, the protein levels of HSP70, acetylated H3 (Ace-H3), galectin-3, and $\beta$-actin were determined by western blot analysis. (F), VPA treatment enhances the levels of Ace-H3, but suppresses galectin-3 production in the BV2 cells with/without LPS exposure. \# $p<0.05$ vs. control. ${ }^{*} p<0.05$ and ${ }^{* *} p<0.01$ indicate comparison with LPStreated BV2 cells with Student's t-test. The data represent mean \pm SEM. 
A

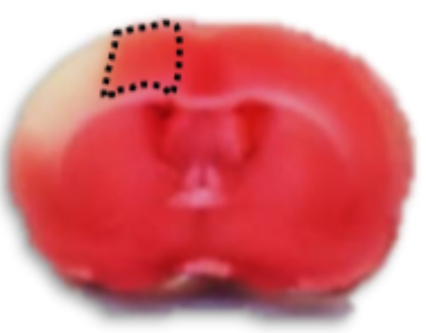

C

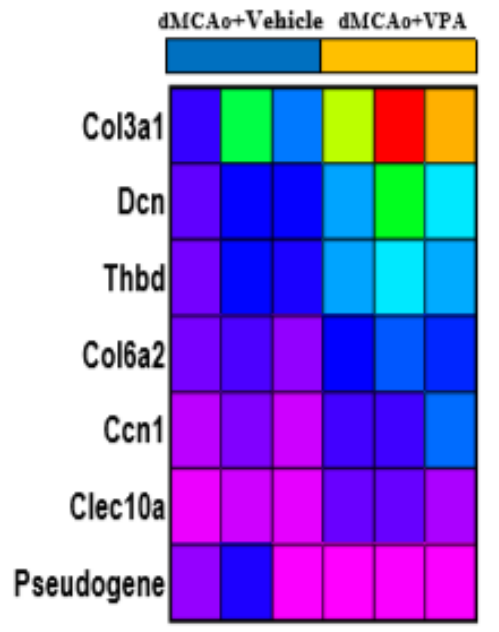

$\mathrm{E}$

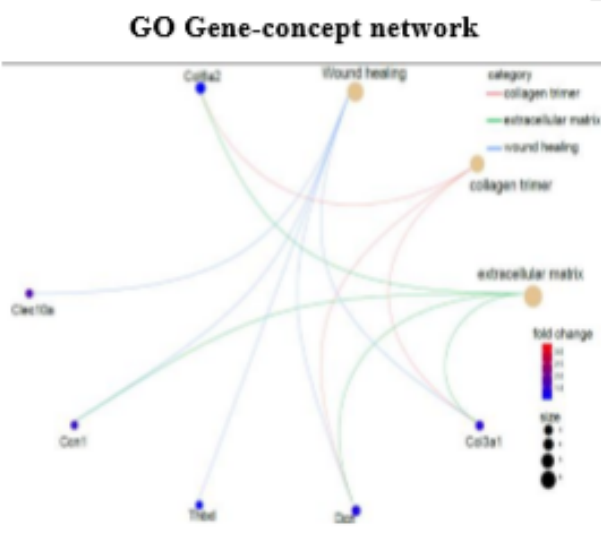

B

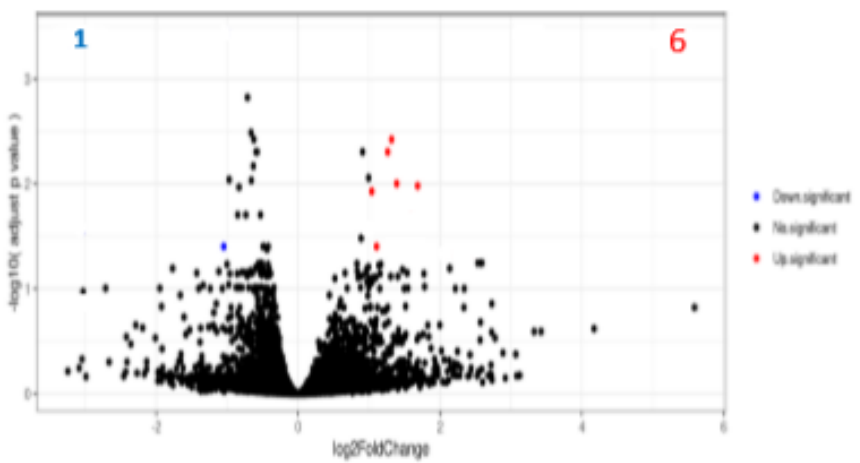

D

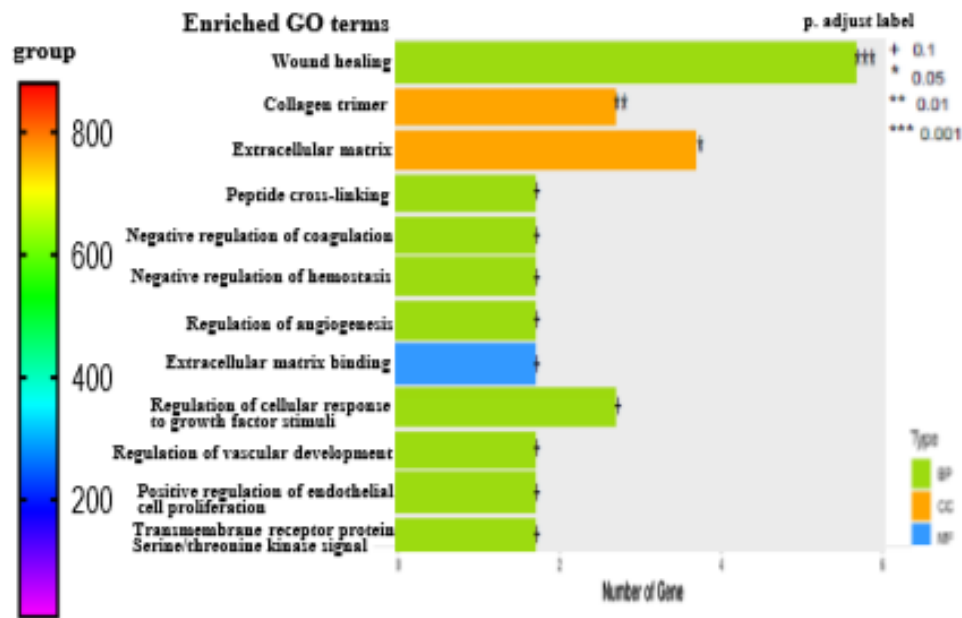

F

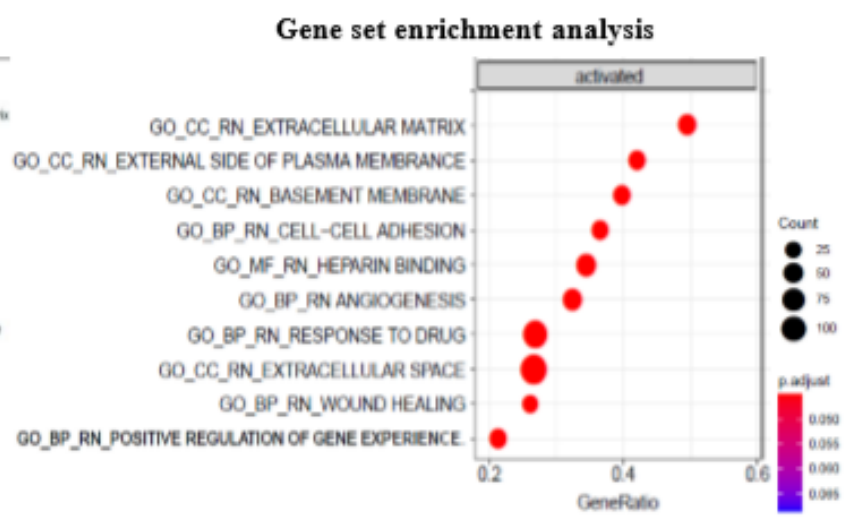

\section{Figure 8}

Post-stroke VPA treatment regulates gene expression in the peri-infarct cortex at 3 days after ischemic brain injury. (A), schematic representation of the brain sections used for the RNA-sequencing experiment. (B), volcano plot comparing the log2fold changes and adjusted p-values of 15446 gene expressions. The red dots indicate genes upregulated (log2fold change $>1$, adjusted p-value $<0.05$ ), the blue dots indicate genes downregulated (log2fold change <-1, adjusted p-value <0.05), and the block dots indicate genes 
with no significant change between dMCAo+vehicle and dMCAo+VPA. (C), heatmap plot of DEGs in periinfarct cortex between dMCAo+vehicle and dMCAo+VPA. (D), GO biological processes, cellular component, and molecular function over-representation analysis based on 7 DEGs. (E), molecular network plot connected using GO over-representation analysis following VPA treatment in a rat model of dMCAo. (F), GO gene set enrichment analysis of genes upregulated by VPA.

A

B

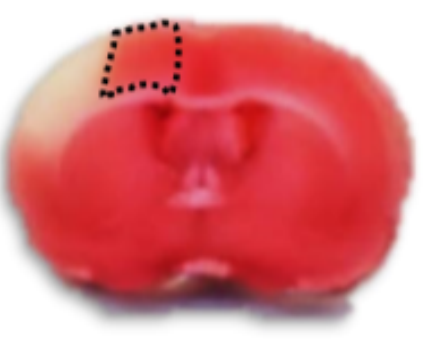

$\mathrm{C}$

$\mathrm{D}$
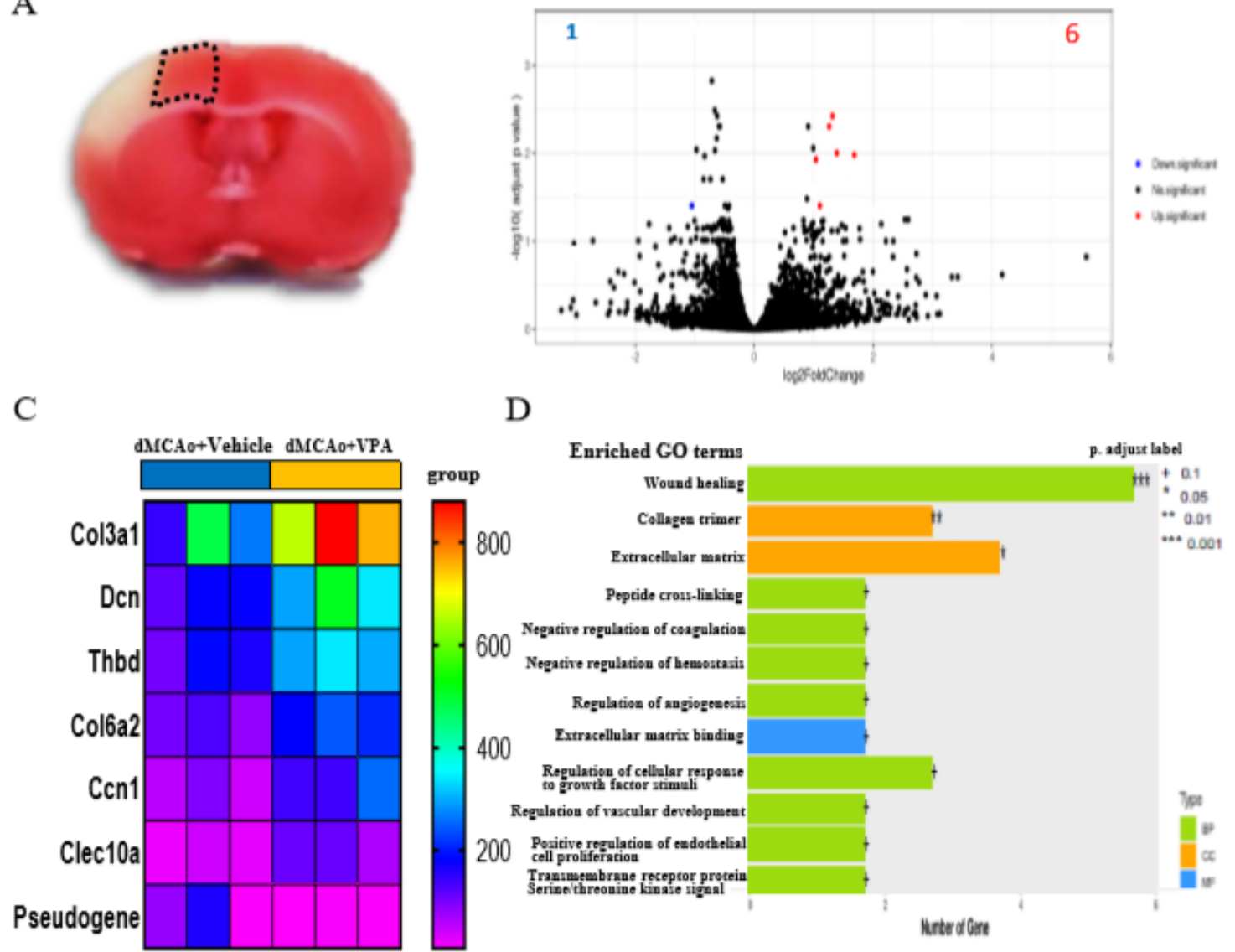

E

$\mathrm{F}$

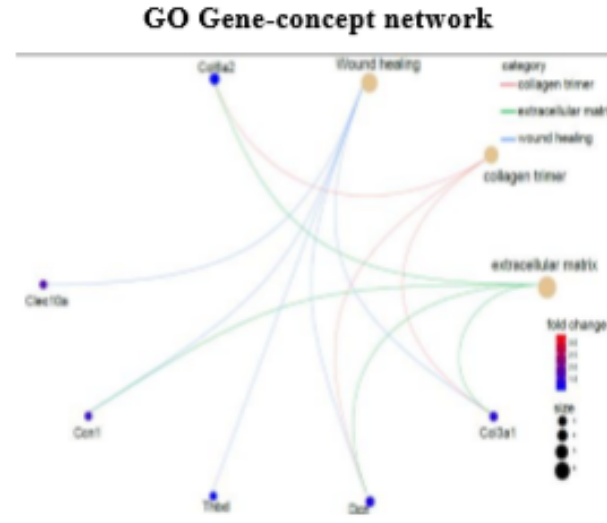

Gene set enrichment analysis

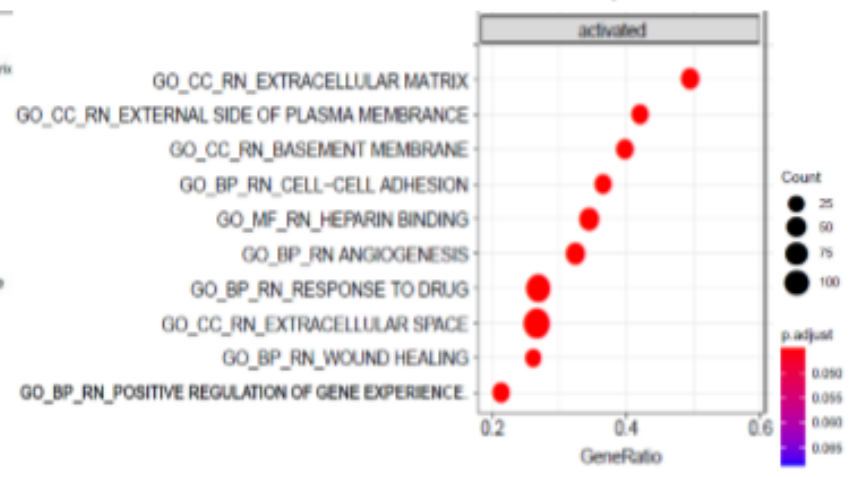

Figure 8 
Post-stroke VPA treatment regulates gene expression in the peri-infarct cortex at 3 days after ischemic brain injury. (A), schematic representation of the brain sections used for the RNA-sequencing experiment. (B), volcano plot comparing the log2fold changes and adjusted $p$-values of 15446 gene expressions. The red dots indicate genes upregulated (log2fold change $>1$, adjusted $p$-value $<0.05$ ), the blue dots indicate genes downregulated (log2fold change $<-1$, adjusted $p$-value $<0.05$ ), and the block dots indicate genes with no significant change between dMCAo+vehicle and dMCAo+VPA. (C), heatmap plot of DEGs in periinfarct cortex between dMCAo+vehicle and dMCAo+VPA. (D), GO biological processes, cellular component, and molecular function over-representation analysis based on 7 DEGs. (E), molecular network plot connected using GO over-representation analysis following VPA treatment in a rat model of dMCAo. (F), GO gene set enrichment analysis of genes upregulated by VPA. 
A

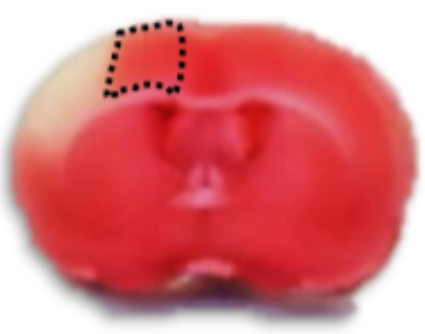

C

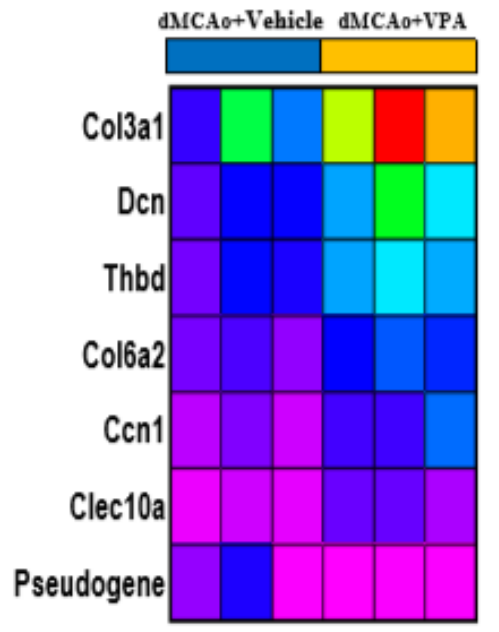

E

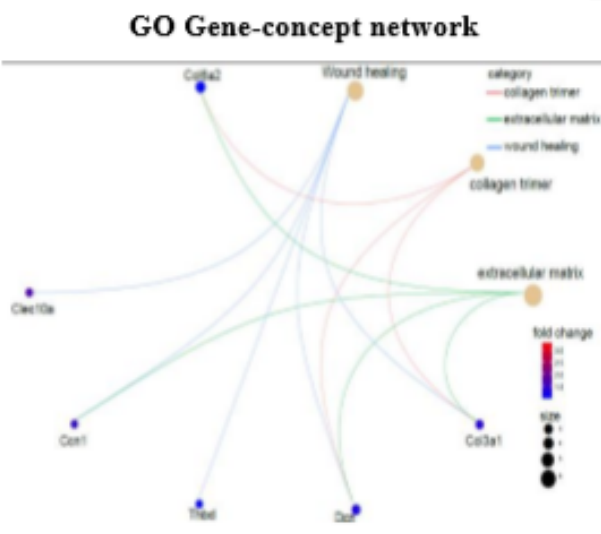

B

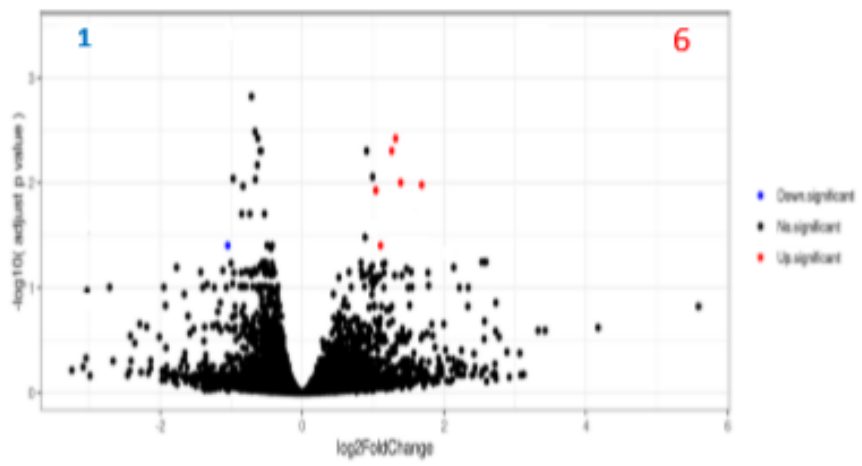

D

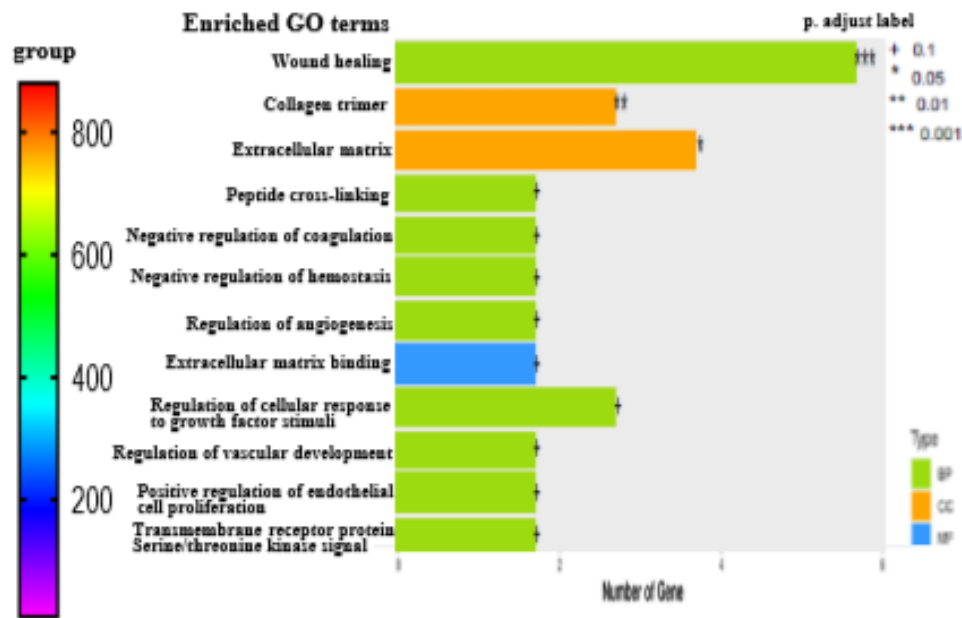

F

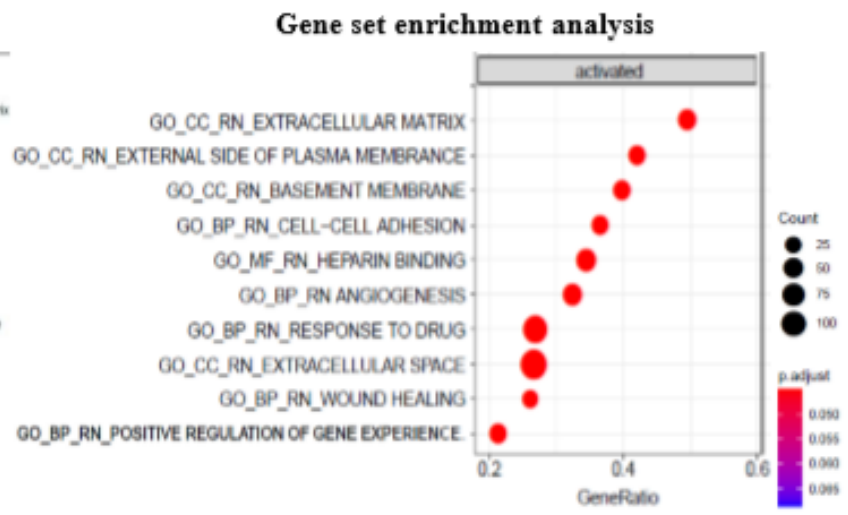

\section{Figure 8}

Post-stroke VPA treatment regulates gene expression in the peri-infarct cortex at 3 days after ischemic brain injury. (A), schematic representation of the brain sections used for the RNA-sequencing experiment. (B), volcano plot comparing the log2fold changes and adjusted p-values of 15446 gene expressions. The red dots indicate genes upregulated (log2fold change $>1$, adjusted p-value $<0.05$ ), the blue dots indicate genes downregulated (log2fold change $<-1$, adjusted p-value $<0.05$ ), and the block dots indicate genes 
with no significant change between dMCAo+vehicle and dMCAo+VPA. (C), heatmap plot of DEGs in periinfarct cortex between dMCAo+vehicle and dMCAo+VPA. (D), GO biological processes, cellular component, and molecular function over-representation analysis based on 7 DEGs. (E), molecular network plot connected using GO over-representation analysis following VPA treatment in a rat model of dMCAo. (F), GO gene set enrichment analysis of genes upregulated by VPA.

A

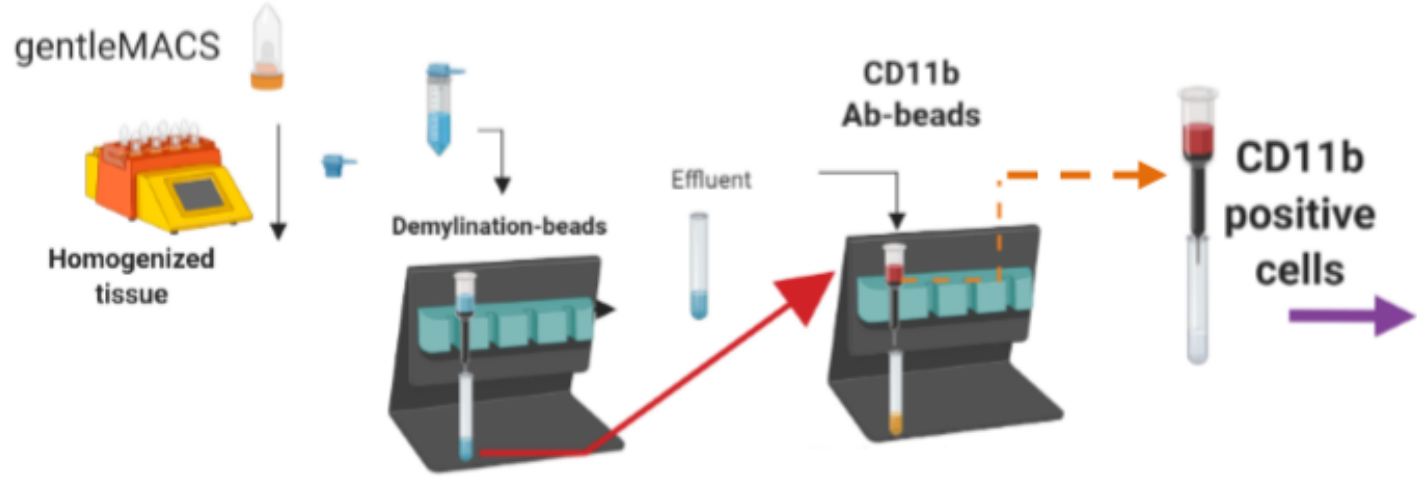

B
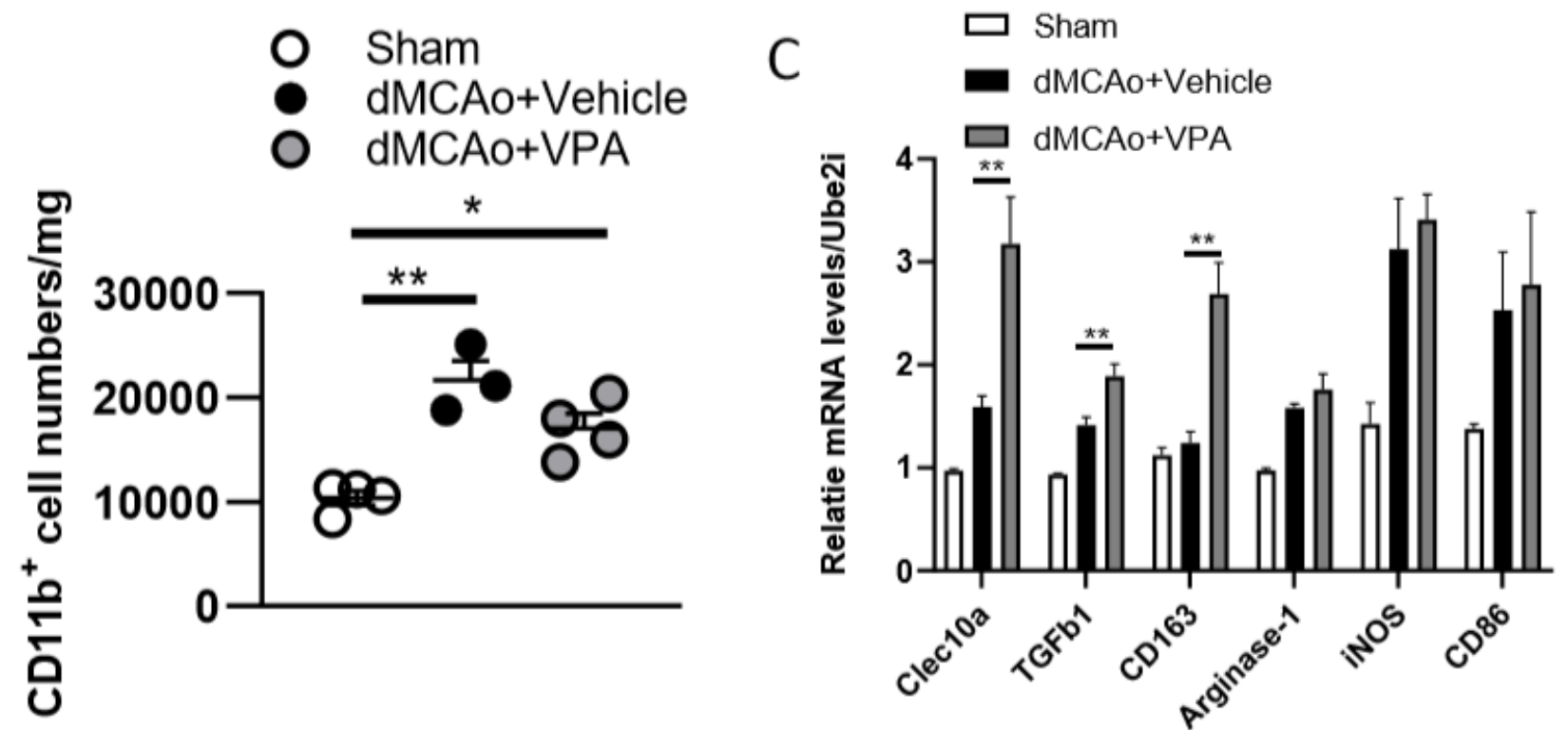

Figure 9

Post-stroke VPA treatment enhances gene expressions of the alternative activated M2 phenotypes. (A), male rats (12 weeks old) were subjected to sham operation or dMCAo surgery with/without VPA treatment. Microglia/macrophages were purified from sham cortex and peri-infarct cortex at day 3 postdMCAo using gentleMACs Dissociator and CD11b antibody-beads. (B), there were significant differences in the number of $\mathrm{CD} 11 \mathrm{~b}$-expressing microglia/macrophages between the sham group and dMCAo groups. (C), qPCR analysis of gene expression of alternative activated M2 phenotype (Clec10a, TGF $\beta 1$, CD163, and Arginase-1) and classical activated M1 phenotype (iNOS and CD86) in the CD11b-expressing microglia/macrophages of sham-operated, dMCAo+vehicle, and dMCAo+VPA groups. ** $\mathrm{P}<0.01$, Student's t-test. $\mathrm{N}=3-4$ per group. The data represent mean \pm SEM. 
A
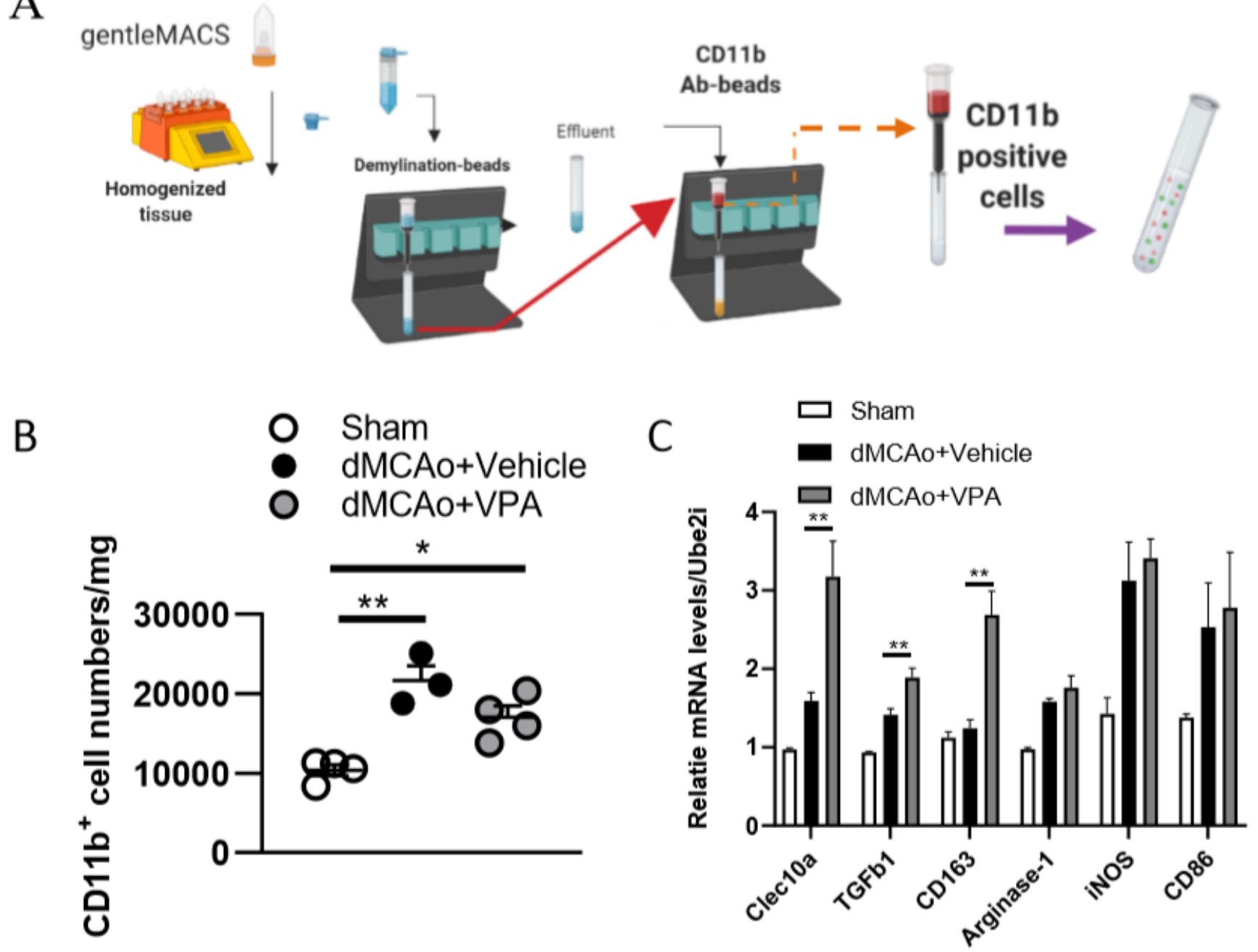

Figure 9

Post-stroke VPA treatment enhances gene expressions of the alternative activated M2 phenotypes. (A), male rats (12 weeks old) were subjected to sham operation or dMCAo surgery with/without VPA treatment. Microglia/macrophages were purified from sham cortex and peri-infarct cortex at day 3 postdMCAo using gentleMACs Dissociator and CD11b antibody-beads. (B), there were significant differences in the number of $C D 11 \mathrm{~b}$-expressing microglia/macrophages between the sham group and dMCAo groups. (C), qPCR analysis of gene expression of alternative activated M2 phenotype (Clec10a, TGF $\beta 1$, CD163, and Arginase-1) and classical activated M1 phenotype (iNOS and CD86) in the CD11b-expressing microglia/macrophages of sham-operated, dMCAo+vehicle, and dMCAo+VPA groups. ${ }^{*} P<0.01$, Student's t-test. $\mathrm{N}=3-4$ per group. The data represent mean \pm SEM. 
A
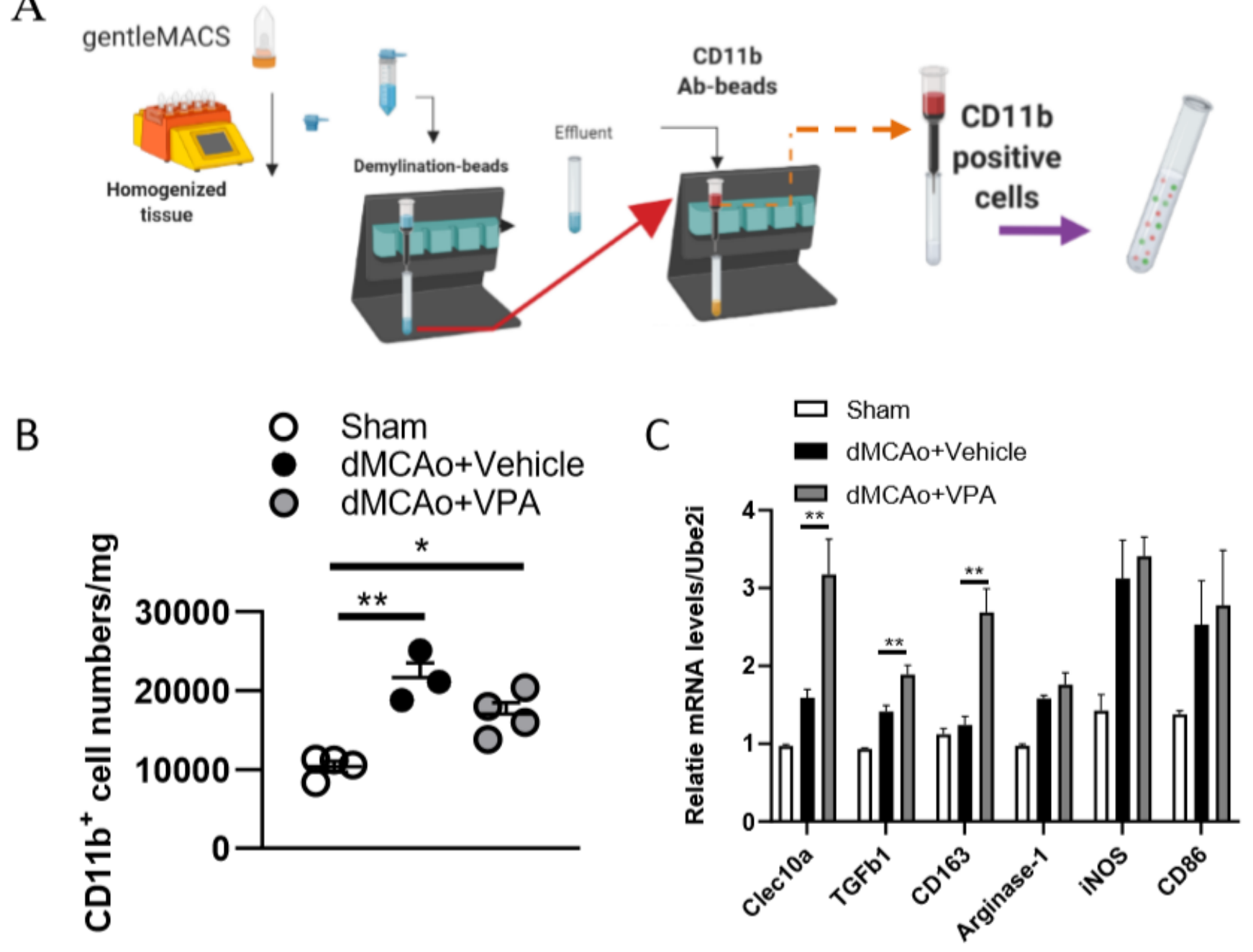

Figure 9

Post-stroke VPA treatment enhances gene expressions of the alternative activated M2 phenotypes. (A), male rats (12 weeks old) were subjected to sham operation or dMCAo surgery with/without VPA treatment. Microglia/macrophages were purified from sham cortex and peri-infarct cortex at day 3 postdMCAo using gentleMACs Dissociator and CD11b antibody-beads. (B), there were significant differences in the number of $C D 11 \mathrm{~b}$-expressing microglia/macrophages between the sham group and dMCAo groups. (C), qPCR analysis of gene expression of alternative activated M2 phenotype (Clec10a, TGF $\beta 1$, CD163, and Arginase-1) and classical activated M1 phenotype (iNOS and CD86) in the CD11b-expressing microglia/macrophages of sham-operated, dMCAo+vehicle, and dMCAo+VPA groups. ${ }^{\star *} P<0.01$, Student's t-test. $\mathrm{N}=3-4$ per group. The data represent mean \pm SEM.

\section{Supplementary Files}

This is a list of supplementary files associated with this preprint. Click to download. 
- additionalfilesTable1.xls

- additionalfilesTable1.xls

- additionalfilesTable1.xls

- additionalfilesTable2.xIs

- additionalfilesTable2.xls

- additionalfilesTable2.xls

- additionalfilesTable3.xls

- additionalfilesTable3.xls

- additionalfilesTable3.xls 